\title{
Evaluating the cost-effectiveness of St. John's wort versus fluoxetine for the treatment of mild to moderate depression
}

Lee Ann Thayer

West Virginia University

Follow this and additional works at: https://researchrepository.wvu.edu/etd

\section{Recommended Citation}

Thayer, Lee Ann, "Evaluating the cost-effectiveness of St. John's wort versus fluoxetine for the treatment of mild to moderate depression" (2003). Graduate Theses, Dissertations, and Problem Reports. 1717. https://researchrepository.wvu.edu/etd/1717

This Thesis is protected by copyright and/or related rights. It has been brought to you by the The Research Repository @ WVU with permission from the rights-holder(s). You are free to use this Thesis in any way that is permitted by the copyright and related rights legislation that applies to your use. For other uses you must obtain permission from the rights-holder(s) directly, unless additional rights are indicated by a Creative Commons license in the record and/ or on the work itself. This Thesis has been accepted for inclusion in WVU Graduate Theses, Dissertations, and Problem Reports collection by an authorized administrator of The Research Repository @ WVU. For more information, please contact researchrepository@mail.wvu.edu. 


\title{
Evaluating the Cost-Effectiveness of St. John's Wort versus Fluoxetine for the Treatment of Mild to Moderate Depression
}

\author{
Lee Ann Thayer \\ Thesis submitted to the School of Pharmacy \\ at West Virginia University \\ in partial fulfillment of the requirements \\ for the degree of \\ Master of Science \\ in \\ Pharmaceutical Systems and Policy \\ Virginia (Ginger) G. Scott, Ph.D., Chair \\ David P. Nau, Ph.D. \\ Eugene H. Makela, Pharm.D. \\ S. Alan Rosenbluth, Ph.D. \\ Department of Pharmaceutical Systems and Policy \\ Morgantown, West Virginia \\ 2003
}

Keywords: St. John's Wort, Hypericum, Fluoxetine, Prozac, Depression, Cost-effectiveness, Decision Analysis

Copyright 2003 Lee Ann Thayer 


\begin{abstract}
Evaluating the Cost-Effectiveness of St. John's Wort versus Fluoxetine for the Treatment of Mild to Moderate Depression
\end{abstract}

\title{
Lee Ann Thayer
}

The objective of this study was to determine the cost-effectiveness of St. John's Wort compared to fluoxetine from a third party perspective for the treatment of mild to moderate depression. Fluoxetine $20 \mathrm{mg}$ daily was compared to St. John's Wort $300 \mathrm{mg}$ thrice daily in a decision tree modeling depression management in the outpatient setting. Probabilities were obtained from a Medline search as well as input from an expert panel of practitioners. Direct medical costs were obtained from medical offices in the Connecticut area with drug and herb cost based on 2002 published data. Several sensitivity analyses were performed to test robustness of the results. The costeffectiveness of St. John's Wort was $\$ 1,788$ per successful treatment compared to $\$ 2,043$ for fluoxetine. Results were sensitive only to large fluctuations in cost of drug or herb therapy. Thus, St. John's Wort is a viable option for patients with mild to moderate depression. 


\section{DEDICATION}

I would like to thank my Committee for the help and encouragement they provided me in completing this project. I would like to especially thank Ginger for her constant encouragement to finish despite my four-year hiatus. I sincerely appreciate the time you put into helping me finish. Thank you for taking over as Chair of the Committee and making sure I stayed on track. I would like to thank David for getting me started and introducing me to pharmacoeconomics. Thank you for your insightful comments and providing me with an excellent foundation of skills.

Completing this thesis was a laborious but important learning experience. It has helped me become a better teacher to the residents and students I precept and a better practitioner serving as a Drug Use Policy specialist. However, I was not always the easiest person to be around while working on this project. I want to thank my friends and family for putting up with me when I was in less than a good mood, to put in mildly. No one understands this more than Brian, my fiancé. Thank you so much for going out of your way to make sure I got things done. Your encouragement and support truly made me feel I could accomplish anything. Thank you again. I love you!

Lastly, I would like to dedicate this thesis to my mother, Jerry Thayer, who passed away January 28, 1997. She was a wonderful person, loving mother and wife, and savvy homemaker. Growing up, she always encouraged me to do my best. I know she would be proud of this accomplishment. Thanks for giving me self-confidence, love and support. I love you very much and miss you dearly. 
ACKNOWLEDGMENTS

$\underline{\text { Committee Members }}$

Virginia (Ginger) G. Scott, Ph.D., Chair

David P. Nau, Ph.D.

Eugene H. Makela, Pharm.D.

S. Alan Rosenbluth, Ph.D.

\section{Expert Panel}

Abe Adel, M.D.

Greg Doyle, M.D.

Carl Liebig, M.D.

Gene Makela, Pharm.D.

Carl Palmer, M.D.

John Vanin, M.D. 


\section{TABLE OF CONTENTS}

Chapter 1 Introduction................................ p.1

Chapter 2 Literature Review.......................... p.17

Chapter 3 Methods...................................p.62

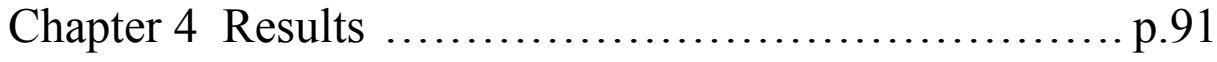

Chapter 5 Discussion and Conclusions................. p.107

References............................................ p.128

Appendix A......................................... p.140

Appendix B........................................ .143

Curriculum Vitae................................. p.144

Figures

3.1 Decision Tree for Treatment of Mild to Moderate Depression.......................................... p.69

Tables

3.1 Base-Case and Range Probability Estimates for St. John's Wort.................................... p.79 3.2 Base-Case and Range Probability Estimates for Fluoxetine ...................................... p. 84 3.3 Direct Medical Costs Associated with Treatment of Depression....................................... 8.86 3.4 Average Retail Price of 30-day supply of St. John's Wort 900mg................................p. 86 3.5 Average Retail Price of 30-day supply of

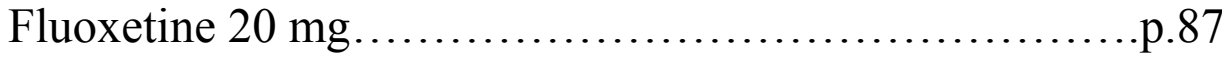

4.1 Total Cost of Care for Fluoxetine....................p.92

4.2 Total Cost of Care for St. John's Wort................ p.93 


\section{TABLE OF CONTENTS}

Graphs

4.1 Sensitivity Analysis on Probability of Success with Fluoxetine...................................... p.95

4.2 Sensitivity Analysis on Probability of Success with St. John's Wort................................ p.96

4.3 Sensitivity Analysis on Probability of Success with St. John's Wort................................ p.97

4.4 Sensitivity Analysis on Cost of one week of

Fluoxetine........................................ p.98

4.5 Sensitivity Analysis on Cost of one week of

St. John's Wort....................................... p.99

4.6 Sensitivity Analysis on Weekly Cost of

Drug Therapy.......................................p. 100

4.7 Sensitivity Analysis on Probability of Remission on St. John's Wort..................................... p.101

4.8 Sensitivity Analysis on Probability of Remission on Fluoxetine.

4.9 Sensitivity Analysis on Probability of Relapse on St. John's Wort. p.103

4.10 Sensitivity Analysis on Probability of Relapse on Fluoxetine.... p.103

4.11 Sensitivity Analysis on Discontinuation of St. John's Wort. p.104

4.12 Sensitivity Analysis on Discontinuation of Fluoxetine. p. 105

4.13 Sensitivity Analysis on Side-effects Requiring Treatment p.106 


\section{CHAPTER 1}

\section{INTRODUCTION}

We are a nation focused on promoting healthy lifestyles, retarding the aging process, and maintaining well-being. Over the last ten years or so, there has been a growing interest in alternatives to traditional medicine such as natural products that claim to enhance health. This is reflected in the rising sales of natural products, referred to as nutraceuticals, and in total expenditures on integrative or alternative medicine. In 1997, Americans doled out $\$ 27$ billion in out-of-pocket expenses for alternative therapy, exceeding out-of-pocket expenses on all physician services in the same year (Eisenburg, 1998). According to Information Resources Incorporated, in 2002 over \$591million was spent on herbal supplements alone (Ferrier, 2002).

The marketing and advertising of these products seem to reflect consumers' interest in a more 'holistic' approach to health care (Astin, 1998). The gamut of botanical and nutraceutical products available is truly extensive ranging from simple conventional food supplements such as peppermint to plant derivatives of echinacea, ginkgo biloba, and ginseng to animal products such as shark cartilage and fish oil. Though appeal in this country seems novel, herbs have been used to treat illnesses for millennia (Cott, 1995). Despite the development of many innovative synthetic medicines, of all prescription drugs products, $25 \%$ are still derived from plants (Batz, 1998). For one specific product, the consumer craze is most evident. 


\section{ST. JOHN'S WORT}

The herb Hypericum perforatum, also known as St. John's Wort, has received a great deal of media attention over the past few years. A yellow flowering plant native to Europe and Asia, Hypericum was brought in the late $18^{\text {th }}$ Century to the Northeastern United States where it has thrived naturally. It has been widely prescribed by German physicians for a range of indications including depressive disorders, minor hemorrhages, wound healing, and even bedwetting in children (Linde et al., 1996; Rao et al., 1991; Vickery, 1981).

In 1994, over 66 million doses were prescribed for the treatment of depression in Germany (De Smet and Nolen, 1996). German sales of Hypericum were \$23 million in 1994 and grew to \$66 million in 1996 (Nash, 1997). For one particular brand of Hypericum, 200,000 prescriptions per month are filled in Germany compared to less than 30,000 for fluoxetine. In fact, prescriptions make up over $80 \%$ of the sales for Hypericum transferring most of the cost of treatment to the health care system (Tyler, 1997). Across Europe, over \$6 billion (United States dollars) were spent in 1998 on products containing the extract (Bilia, 2002). Its popularity continues to grow in the United States, though not quite as rapidly in the past year as questions of efficacy and quality arise.

Uses of the herb date back to the ancient times of Hippocrates and Galen who wrote of various medicinal properties such as the wound-healing, diuretic, and antiviral effects Hypericum possesses. Among American herbalists, most notably John King, the 
documentation of Hypericum as an antidepressant, diuretic, and sedative continued (King, 1866). Other countries that commonly use the herb for medicinal purposes include Czechoslovakia, France, Poland, Romania, and Russia, which each list in their respective pharmacopoeias (Hobbs, 1989; Reynolds, 1993). It is the most wellresearched and extensively used herbal antidepressant known (Bloomfield and McWilliams, 1996). Media coverage has also been extensive with articles in popular newspapers, magazines, and even television programs such as CNN and ABC's 20/20 reporting the potential medical breakthrough of a safer, cheaper, and effective alternative to prescription antidepressants.

With the side-effect profile being much more safe and mild compared to prescription alternatives, Dr. Michael Jenike, editor of the Journal of Geriatric Psychiatry and Neurology and professor at Harvard Medical School, reports Hypericum is a 'particularly attractive choice' for elderly patients (Bloomfield and McWilliams, 1996). It is noteworthy that individuals over the age of sixty-five are more likely than the general population to suffer from a depressive disorder and much more vulnerable to side-effects and drug interactions.

Private manufacturers of St. John's Wort have taken hold of the praises from the medical community and have spread them like wildfire, adding some extras along the way. Preparations of the herb can be found in combination with other ingredients such as ephedra and cascara claiming to be a natural version of the once available prescription combination, PhenFen. There is no evidence that the herb works as an appetite 
suppressant, but the advertising alone has been enough to spark a buy-in from the diet industry. Over half of the dieters at all NutriSystem weight loss centers, representing almost 250,000 people, reported taking St. John's Wort as a weight-loss supplement (Associated Press, 1997).

Consumers too have accepted these claims with open arms. Sales of products containing Hypericum have increased dramatically from \$143,446 in 1996 to \$29.4 million in 1997 according to Information Resources, Inc based in Chicago (Huff, 1998). By 1998, this figure grew exponentially to over $\$ 5$ billion (Brevoort, 1998). Certainly the boom in advertising in addition to the increased availability of products has played a role in the blossoming sales. However, most recently, sales have hit a plateau. Much of this has been blamed on media attention questioning the effectiveness of the herb along with disputes of the quality of many of the marketed products. Others point to the overall decline in the economy blunting sales of any type of luxury item (Ferrier, 2002).

The once uninterested pharmaceutical giants quickly joined in the niche market, manufacturing a variety of herbal supplements. GlaxoSmithKline markets St. John's Wort along with a whole line of German herbals called Abtei. Following suit, Bayer Pharmaceutical Consumer Product's division developed a line of vitamin supplements that contain some of the most popular herbs such as Echinacea, Ginsing, Ginko, and St. John's Wort. Other companies have attempted to gain prescription status for some of these preparations and are currently seeking approval from the Food and Drug Administration (FDA) (Wilke, 1997). 
An article in Health magazine (Benedict, 1998) reports that even people who aren't depressed are taking the herb. With the type of statements manufacturers are making, it's not surprising to hear this. Larry King claims that "clinically tested St. John's Wort keeps him balanced emotionally' in an advertisement on the radio for a combination product containing Hypericum. Commonly referred to as the 'Natural Prozac', products claim all the benefits with none of the adverse effects. It's the new rage in curing what ails you.

So why are manufacturers allowed to make unsubstantiated claims that have not been tested or may not have any medical evidence to back them up? The reason is due to the relaxed regulations involved with the marketing of these products. Botanicals, such as herbs, are considered dietary supplements and not drugs. Therefore, the marketing of these products is not regulated with the same rigor as prescription or over-the-counter drug products.

Herbs are not subject to FDA regulations but fall under the guidelines of the Diet Safety Health and Education Act (DSHEA) of 1973. Under this act, a herbal product is regulated as a food supplement and hence does not require proof of safety to be marketed. However, the DSHEA does restrict manufacturers on the types of claims that may be made regarding the product (Batz, 1998).

All claims that are made on the label must bear the words, "This statement has not been evaluated by the FDA. This product is not intended to diagnose, treat, cure, or prevent 
any disease." Only 'structure-function' or 'well-being' claims can be made about the product. In order for a product to be removed by the FDA, they must prove it to be unsafe or prove a claim false (Batz, 1998). The FDA has been trying to develop current good manufacturing practices (GMPs) for dietary supplement manufacturers since 1994 (Cooperman and Obermeyer, 2002). The standards are planned to be modeled after the food industry rather than the drug manufacturers' GMPs. As this is a difficult and timeconsuming process, it is expected to take several more years before it is implemented and becomes law.

In other countries, such as Germany, there are finer, more well-established guidelines for which products may be used as therapeutic treatments for illness. Again, these regulations are not as rigorous as the FDA's New Drug Application process in the United States, but it does establish criteria for which the product may be used. The German Commission $\mathrm{E}$ is a division of the German regulating agency similar to the United States FDA. They have developed a monograph system that evaluates the use of herbal products for medicinal use. Absolute proof of safety is required for approval and relative proof of efficacy through clinical trials or historical use (Batz, 1998).

Commission E is continually developing more monographs. St. John's Wort was one of the first established. Having the monograph system as a therapeutic guide, physicians in Germany are very knowledgeable and confident about prescribing herbs for chronic conditions. In 1999, the United States Pharmacopeia (USP) published a monograph and 
established recommended potency of specific constituents based on High Pressure Liquid Chromotography analysis (USP, 1999).

Whether this growth in alternative medicine has been spurred by a general disenchantment with traditional therapies or simply a desire to try a more natural, holistic approach to health care, it is a reflection of the desires and interests of consumers. The dearth of information available for many of these alternatives emphasizes the need to cautiously scrutinize and judge the potential of these products as viable alternatives. The public and healthcare community needs an unbiased approach to be taken to accurately assess the role these botanicals have in health care. Cost-effectiveness analyses can serve to do this along with providing information to businesses wishing to meet the needs of their customers such as Health Maintenance Organizations (HMO) and other third party payers of healthcare.

In the midst of all the media hype, there does seem to be a legitimate use of the herb called St. John's Wort. The documented use in Germany, more recent published American trials, and expert opinion from physicians in the United States suggests great potential of this herb for the treatment of mild to moderate depression. Since the cost is about one-tenth to one-fifth that of its prescription comparisons, savings to the patient and the health care system could be tremendous.

However, mere acquisition cost is not enough to comprehensively assess the impact a new treatment for depression can have on society and the health care system in general. 
Therefore, this study shall focus on the relative cost-effectiveness of St. John's Wort versus fluoxetine, one of many prescriptive comparators, in the treatment of mild to moderate depression. This type of analysis can provide much more information to patients, providers and payers of health care than clinical trials measuring efficacy alone. Nonetheless, many factors must be considered to construct a model representative of all costs and consequences.

One must understand the complexity of the disorder and the extent to which it affects individuals in this country. Furthermore, treatment should be assessed in relative terms. It makes sense to compare the potential therapy with a gold standard, such as one of the selective seratonin reuptake inhibitors (SSRIs) currently available. The following two sections shall provide the information needed to justify the comparison of St. John's Wort to fluoxetine in the treatment of mild to moderate depression.

\section{DEPRESSION}

Approximately 6.7 million women (6.5\%) and 3.2 million men (3.3\%) aged 18 years and over have a depressive disorder in the United States in a given year (Narrow, 1998). Of course the true prevalence of depression is not known partly due to the characteristics and social stigma surrounding the condition. Most cases are not diagnosed by psychiatrists but by general practitioners, making it one of the most frequently presented conditions in the primary care setting. Approximately $25 \%$ of patients seen in the primary care setting have some form of depression (Henry and Rivas, 1997). Paradoxically, primary care and 
other non-psychiatric practitioners often under-diagnose and under-treat depressive symptoms despite their common occurrence.

Diagnostic criteria according to the Diagnostic and Statistical Manual (DSM-IV) for a major depressive episode include five or more of the following symptoms lasting at least two weeks or more with depressed mood or anhedonia being one of the five symptoms (DSM-IV, 1997):

- Depressed Mood

- Loss of interest or pleasure in usual activities (anhedonia)

- Significant weight loss or gain

- Insomnia or hypersomnia

- Psychomotor agitation and retardation

- Fatigue

- Feelings of worthlessness or guilt

- Diminished ability to think or concentrate

- Recurrent thoughts of death or suicidal ideation

Along with eliciting the appropriate signs, symptoms, and history of illness, knowledge of associated risk factors is a key determinant in the detection and diagnosis of depression. The primary risk factors for depression are (Depression Guideline Panel, 1993):

- Prior episodes of depression

- Family history of depressive disorder

- Prior suicide attempts

- Female gender

- Age of onset under 40

- Postpartum period

- Medical co-morbidity

- Lack of social support

- Stressful life event

- Current alcohol or substance abuse 
As stated above, a prior depressive episode represents a significant risk factor for recurrence of depression. In fact, an individual diagnosed with depression has a $50 \%$ chance of suffering a subsequent episode. The rates increase dramatically with the number of reported episodes, up to $90 \%$ with three or more episodes (National Institute of Mental Health Consensus Development Conference, 1985). Relapse is also highly associated with medication adherence. Individuals treated with adequate doses of an antidepressant that experience remission of symptoms are more likely to remain in remission if maintenance therapy is continued for a period of at least six months (Henry and Rivas, 1997).

The more the complexity of this disorder is understood, the more apparent becomes the debilitating nature of depression. Individuals frequently somaticise symptoms causing chronic pain, gastrointestinal disturbances such as peptic ulcers, and a host of other conditions. Hospital costs are usually $35 \%$ greater and visits are twice as long for depressed patients with physical illnesses compared to patients of similar severity without depression (Henry and Rivas, 1997).

Due to the nature of depression combined with the associated stigma, frequently, depressed persons withdraw from society and are unable to engage in daily activities including going to work. This represents another significant cost to the individual through lost wages as well as to society through a loss of productivity. Adding another cloud to the dismal picture already painted is the high rate of suicide accompanying major depression with a ten to $15 \%$ mortality rate (Henry and Rivas, 1997). According to 
a study conducted by the World Health Organization along with Harvard University, the fourth leading cause of disability worldwide can be attributed to major depression (Murray, 1996).

Pressures to decrease medical costs have put a tremendous burden on the health care system and mental health services in particular. Fewer HMO's are willing to pay for specialized psychiatric treatment, and many plans carve out mental health from general health benefits. With depression being one of the top ten reasons an individual consults a family physician, primary care practitioners are handed the challenge of providing quality care at an affordable cost. The most widely prescribed classes of antidepressants in the United States are the SSRIs. One of the most commonly prescribed drugs in this class is fluoxetine (Prozac).

Annual costs of depression in the United States were estimated at $\$ 43.7$ billion in 1990 including treatment, disability, and loss of productivity (Colchamiro, 1998). The costs in total are greater than that of ulcers, diabetes, arthritis, or hypertension (Tyler, 1997). Pharmacotherapy can be quite costly especially when newer agents such as the SSRIs or combination seratonin/norepinephrine re-uptake inhibitors (SNRIs) are used. Costs for these therapies can run as high as seven dollars per day. Less expensive alternatives do exist but sometimes at the expense of bothersome and toxic effects. Also, these compounds may not be appropriate for all types of depression. 


\section{FLUOXETINE}

Since it's introduction over 10 years ago, fluoxetine has been available to psychiatrists, primary care physicians, and other non-psychiatric physicians as effective phamacotherapy for patients with depression. It's arrival undoubtedly made its mark in the prescribing arena. Evidence of this comes from impressive standings as the number one prescribed antidepressant and the second top selling drug in the country when considering all prescription drugs in 1998. Manufacturer Eli Lilly \& Co. boasts sales of greater than $\$ 2$ billion per year (Tyler, 1997).

Its popularity among prescribers is due to a number of clinical reasons. Tricyclic antidepressants (TCAs), prior to fluoxetine's market approval, were the cornerstone of antidepressant therapy for decades. Fluoxetine presented an incredible advantage over this class of compounds offering a wide therapeutic range with little toxicity in overdose. The side-effect profile is more benign and fewer patients have been shown to discontinue therapy compared to TCAs (Stokes and Holtz, 1997). In light of this, fluoxetine is thought to enhance medication adherence during maintenance therapy and thus, improve response and decrease chance of relapse.

One advantage of fluoxetine over other SSRIs is the relatively long half-life it has (four to six days with chronic administration). This enables patients to have a convenient once a day dosing schedule and the titration that is necessary with many of the TCAs is not a factor for patients beginning therapy with fluoxetine. A new once-weekly formulation 
was marketed in October, 2002, potentially increasing adherence and convenience even more.

As far as acquisition cost of the drug, Prozac was one of the most expensive antidepressants on the market. However, the patent expired in October, 2001, and generic fluoxetine entered the market. Its cost is about one-third the cost of the brand. Even with the generic now available, the cost of treatment is still more expensive than that of St. John's Wort. Hence the question still remains. Is fluoxetine, representing the class of SSRIs, the most cost-effective drug to treat mild to moderate depression?

\section{STATEMENT OF THE PROBLEM}

With the rising costs of healthcare, it is becoming imperative to use treatments that have proven value. One way to determine this value is through cost-effectiveness analyses. Depression, a costly disorder physically, financially, and emotionally, impacts our society greatly. With $9.5 \%$ of the population age 18 and older being affected in a given year, there is a need for effective treatments that are well tolerated (Regier, 1993). Determining the relative cost-effectiveness of different therapies for depression is important due to the prevalence and high costs associated with its treatment.

Selective seratonin reuptake inhibitors such as fluoxetine have made substantial contributions to the existing antidepressant treatments by offering superior safety, 
convenient dosing, and in some ways a more tolerable side-effect profile. Unfortunately, the cost of these drugs is very high, and expense of treatment can be a major hurdle for all involved in the healthcare system. If herbal therapies were as safe and effective as fluoxetine for the treatment of depression, but were less expensive, patients could be treated more efficiently or cost-effectively.

Global and economic pressures have created a renewed interest in natural product research that has provided us with extensive documentation and clinical information on certain herbs. Clinical trials conducted on St. John's Wort document its efficacy in the treatment of mild to moderately severe depression, and associated side-effects are almost nonexistent. The cost of the herb is about one-fifth that of the brand Prozac. However, a direct and comprehensive comparison of the cost-effectiveness of fluoxetine with St. John's Wort has not been conducted. Such a comparison would be useful in guiding formulary decisions and treatment algorithms for depression.

\section{GOALS AND OBJECTIVES}

The goal of this study is to determine the cost-effectiveness (cost per successfully treated patient) of St. John's Wort compared to fluoxetine in the treatment of mild to moderate depression for a treatment period of one year. The perspective of the study shall be the third party payer or managed care point of view. This goal will be accomplished by the following objectives: 
Objective A. Calculate the expected cost associated with the treatment of mild to moderate depression using fluoxetine at the initial dose of $20 \mathrm{mg}$ once a day.

Objective B. Calculate the expected cost associated with the treatment of mild to moderate depression using St. John's Wort at the initial dose of $300 \mathrm{mg}$ three times a day.

Objective C. Assess the overall cost per successfully treated patient (cost-effective ratio) who responds to St. John's Wort.

Objective D. Assess the overall cost per successfully treated patient (cost-effective ratio) who responds to fluoxetine.

Objective E. Assess the incremental cost-effectiveness ratio for St. John's Wort compared to fluoxetine.

Objective F. Assess the robustness of the findings by conducting sensitivity analyses.

\section{SIGNIFICANCE OF THE STUDY}

St. John's Wort has been used to treat depression, which is a very prevalent, disabling, and costly disorder in this country. The current standard treatment for patients with mild 
to moderate depression is costly and can cause troublesome side-effects that may decrease quality of life and may influence discontinuation of the medication. It is worthwhile to compare the relative effectiveness and cost of this commonly used herbal treatment to fluoxetine, a drug from the class most often used for depression today. It may represent a new effective treatment choice for patients suffering from this disorder and concurrently decrease the costs of care for this sizable population.

Improvements in pharmacotherapy have been incremental over the last decade. Selected populations may benefit extensively from the safety and tolerability of St. John's Wort. Side-effects such as anxiety and sexual dysfunction may no longer plague those trying to fight the battle of depression. Additionally, those with no health insurance may no longer have the hurdle of cost of therapy to overcome.

More informed decisions can be made in the light of the information this study can provide. The model can serve to integrate clinical data and expert opinion that is currently available until more definitive studies are available. Managed care organizations can objectively assess the potential of St. John's Wort as a formulary drug and its potential impact on the effectiveness and costs of treating patients with depression. 


\section{CHAPTER 2}

\section{LITERATURE REVIEW}

\section{INTRODUCTION}

The literature that focuses on depression and issues concerning pharmacologic treatment is quite extensive. With the substantial impact this disease imposes upon the world, such interest is no surprise. Depression has been listed as the number one cause of disability in the world (Murray, 1996). Much has been learned about the epidemiology of

depression such as risk factors, diagnostic criteria, prevalence, treatment protocols, and relapse rates. The vast gamut of research in this area has aided in the development of clinical treatment guidelines established by the Agency for Healthcare Research and Quality (formerly the Agency for Healthcare Policy and Research (AHCPR, 1999). Progressive research has also lead to the discovery of more compounds possessing antidepressant properties.

With the market availability of these new antidepressants, controversies arise over the actual benefit in treating the disorder. Given that new drugs added to the list of prescriptive antidepressants have a much higher acquisition cost relative to their older counterparts, budget constraints force policy makers to ask questions about how and when to use these therapies. Concerns such as these have been addressed in the literature. Many researchers have tried to evaluate the impact that choice of drug treatment has on total cost of care by utilizing techniques of pharmacoeconomics. 
In general, pharmacoeconomic evaluations include any study that examines costs and consequences of alternatives (Bootman, 1996). Examples of these are cost-benefit, costutility, cost-minimization, and cost-effective analyses. A variety of approaches can be used to conduct studies such as these. A method commonly used since the early 1970 s is economic modeling (Lusted, 1971). There are three basic modeling approaches that a researcher can take. These are epidemiological, Markov, and decision analysis modeling (Drummond, 1997). Some basic information on each of these approaches follows with a more in-depth discussion of decision analysis presented in Chapter 3.

\section{ECONOMIC MODELING TECHNIQUES}

Epidemiological models are commonly used when intermediate outcomes are measured as a proxy for final outcomes (Drummond, 1997). Often times a relationship must be established between the intermediate and final outcomes in order for a meaningful economic evaluation to be done. Biological measures such as blood pressure or serum cholesterol are often used as indicators or predictors of coronary artery disease (CAD) in clinical trials. By using epidemiological data combined with modeling techniques, risk rates can be established to link the biological marker to the final outcome of CAD.

In cases where the disease or treatment is characterized by chronically recurrent events or a number of different health states, an alternative approach is Markov modeling. Markov models incorporate probabilities of transition between different health states for each cycle specified by the model (Drummond, 1997). The cycle has a defined time frame, and a defined number of times it is repeated. The cycle length in the model often differs 
from the evaluation length in the clinical study where probability estimates are derived. This necessitates the calculation of hazard rates to estimate transition probabilities.

The third method, decision analysis modeling, has been used extensively in health care (Weinstein and Fineberg, 1980). It involves a process of delineating all possible costs and outcomes of two or more alternatives over a specified time period by way of a decision tree. The tree enables visualization of different choices and the associated chances (uncertainty) represented in the clinical scenario and calculates the optimal alternative under the chosen assumptions (Lawler, 1995).

In order to construct such a model, certain elements must be addressed. Perspective of the study, length of treatment, and model assumptions must be clearly described. In order to analyze the model, effectiveness, safety, and cost data for each therapeutic alternative must be evaluated. Additionally, the classification of major depression and associated treatment practice patterns must be taken into consideration. The following sections discuss these issues required to conduct this economic evaluation.

\section{MAJOR DEPRESSIVE DISORDER}

Major Depressive Disorder (MDD) is a growing health concern in the United States with approximately 6 to $8 \%$ of all primary care patients suffering from the disorder (AHCPR, 1999). The economic impact caused by social and physical dysfunction has been estimated to be greater than that of many chronic medical conditions such as diabetes and heart disease. In 1990, total cost of MDD was $\$ 43.7$ billion according to a study 
examining the financial burden due to loss of productivity and absenteeism, suicides, and treatment and rehabilitation (Greenberg, 1993).

Due to a number of factors indigenous to the disorder, MDD has gone unrecognized and hence untreated in many individuals. In many cases, depression is mistaken for anxiety; in fact, only about half the patients diagnosed as having a depressive or an anxiety disorder exhibit relatively pure syndromes of one type or the other (Brown, 1997). Additionally, although they are separate diagnostic categories, other psychiatric medical disorders may exist with depression such as bipolar illness or psychoses. In light of this, efforts are being made to improve the diagnostic acuity of physicians in primary care where more often than in any other setting, the associated symptoms are presented (Bilia, 2002).

The diagnostic criteria for MDD include five or more of the following symptoms to be present most of the day, nearly daily, for a period of two weeks or more. At least one of the first two listed symptoms must be present (Williams, 2000):

1. Depressed Mood.

2. Loss of interest or pleasure in usual activities (anhedonia).

3. Significant weight loss or gain.

4. Insomnia or hypersomnia.

5. Psychomotor agitation and retardation.

6. Fatigue.

7. Feelings of worthlessness or guilt.

8. Diminished ability to think or concentrate.

9. Recurrent thoughts of death or suicidal ideation. 
Another phenomenon existing with this disorder is the alarming rate of relapse. An individual diagnosed with depression has a $50 \%$ chance of suffering a subsequent episode. The rate increases drastically with the number of reported episodes, up to a $90 \%$ chance of relapse with three or more episodes (NIH Consensus Development Conference, 1991) but can be minimized with continued use of an effective antidepressant. This verity has lead to the recommendation of continuing antidepressant therapy for at least 16 weeks beyond initial recovery for a total of six to 12 months of treatment (Williams, 2000).

Selecting the antidepressant, once a diagnosis of MDD has been made is based on a number of factors. Issues involving safety and effectiveness of the drug are of primary concern in the decision making process. Before there is any established use of a drug, this information predominantly comes from published randomized clinical trials where efficacy can be measured by severity rating scales.

A diagnosis of depression categorizes the medical illness but does not delineate the level of severity. There are three main levels under which depression may fall: severe, moderate, and mild depression. About $75 \%$ of all depressive illnesses are of mild to moderate severity (Muller and Voltz, 1996). A number of instruments are available to assess the severity of depression, which is essential when investigating efficacy of treatment modalities. 


\section{DEPRESSION RATING SCALES}

The Hamilton Depression Rating Scale (HAMD) is an observer-rated depression scale created specifically for the purpose of assessing severity level. It was developed in 1960 by Max Hamilton. The author intended its use only for patients already diagnosed with major depressive disorder.

There are several versions available today, but the original is a 21-item scale. A 31, 25, 24 and 17-item scale have been reported with the original and abridged versions being the more commonly used. A fairly wide range of symptoms is covered by the original version including the core symptoms of depression, hypochondriasis, loss of insight, obsessive-compulsive features, anxiety, and psychosocial impairment. Hamilton stated the reason for such a comprehensive coverage of symptoms was due to the fact that these were the features that usually presented in patients diagnosed with depression (Snaith, 1996).

Typically, an improvement in a patient's severity or a response to treatment is defined as a $50 \%$ decrease in baseline score or an endpoint score $\leq 10$. Through various studies conducted on non-clinical samples, absolute HAMD scores between six and eight are considered to be the normal range for healthy populations (Fawcett, 1997). Therefore, total remission of depressive symptoms or optimal response to therapy can be determined based on this range of scores. 
Currently, the HAMD is the most prevalent scale used in psychiatric research for the severity of depression assessment (Snaith, 1996). However, there are a number of other scales that have been used to evaluate therapeutic efficacy. Some examples of these include the Bech-Rafaelsen Melancholia Scale (BRMS), the Montgomery-Asberg Depression Rating Scale (MADRS), the Depression Anxiety and Stress Scale (DASS), the Beck Depression Inventory (BDI) and the Clinical Global Impressions index (CGI).

The BRMS is a self-rated scale used to assess severity of symptoms. Unlike the HAMD, it can also be used to diagnose depression. It consists of eleven items with five levels of scoring. While the HAMD covers a broad range of emotional symptoms, the BRMS covers all core symptoms of depression and only a limited number of questions are devoted to anxiety. Similar to the HAMD, the BRMS has demonstrated excellent reliability measures and valid interpretations of severity assessment in depressed patients (Maier, 1988).

The MADRS was initially devised to be more sensitive toward change in severity during treatment than the HAMD. It is also a self-report instrument. It contains ten items and seven levels for the scoring of those items. Additionally, it has a much narrower focus than the previous two scales discussed. There is sufficient evidence demonstrating its reliability and validity for the purpose of depression severity rating (Bech, 1986).

The Depression Anxiety and Stress Scale (DASS) consists of 42 negative emotional symptoms that is self-rated. This instrument differs from the others specifically based on 
the fact that three distinct domains are tapped compare to the more common two domain structure of depression and anxiety. The factors contained in the depression scale relate to a loss of self-esteem and incentive and are associated with a low perceived probability of attaining life goals of significance for the individual (Lovibond, 1995).

The BDI is most often used for psychotherapy studies. It is a standardized scale rated by the patient on functional and emotional outcomes. Studies have shown that scores obtained from this scale correlate with those obtained by the HAMD.

The CGI is an observer rated instrument that has three indices of measurement. Not only does this instrument assess severity of illness, but it also assesses global improvement and efficacy of therapy. The CGI is commonly used to represent global improvement and is used in combination with one or more of the aforementioned scales. Results are presented as nominal data such as "improved" or "much improved" (APA, 2000).

Each of these scales as well as numerous others not mentioned here allows for the assessment of severity of symptoms in patients diagnosed with depression. Some scales are able to tap into different domains of the disorder better than others. However, most of the studies in which these scales were used did a good job of assessing therapeutic response rates as evidenced by similar findings for effectiveness rates.

When similar results are obtained by multiple scales that assess severity, it becomes apparent that the data is more robust than simply using results of one scale. It is 
frequently necessary to combine results of studies that have used different instruments to assess response to therapy. Meta-analysis can be a useful tool to capitalize on external validity, which is advantageous for studies such as cost-effective analyses whose results tend to have policy or program implications (Luce and Simpson, 1995).

\section{PHARMACOTHERAPY FOR MAJOR DEPRESSION}

Pharmacotherapy is the only proven method for treating all levels of severity of MDD (Stokes and Holtz, 1997). There are a number of options available on the market today. The first compounds discovered make up the class referred to as tricyclic antidepressants (TCAs). The drugs falling under this heading are extensive including imipramine, amitriptyline, and desipramine. These drugs have been a mainstay in depression therapy. With over forty years of clinical use, their effectiveness in treating depressive symptoms is well documented.

The empirical research on efficacy does not favor any particular TCA over another in depressed populations. Approximately 50 to $80 \%$ of depressed patients have responded to tricyclic therapy, with the most extensive documentation on amitriptyline and imipramine (Einarson, 1995; Depression Guideline Panel, 1993). The mechanisms by which these drugs exert their effect cause a host of troublesome side-effects and have long been a barrier to symptom control. Compliance problems occur in 15 to $20 \%$ of patients on TCA regimens. Anticholinergic effects such as dry mouth and sedation along with potentially fatal cardiotoxic properties have precipitated the search for better tolerated, safer alternatives. 
This search lead to the discovery of a class of compounds commonly referred to as selective seratonin reuptake inhibitors (SSRIs). To date, there are six marketed compounds in this category: fluoxetine $(\operatorname{Prozac} \AA)$, paroxetine $(\operatorname{Paxil} ®)$, sertraline $\left(\right.$ Zoloft $\left.{ }^{\circledR}\right)$, fluvoxamine $\left(\operatorname{Luvox}{ }^{\circledR}\right)$, citalopram $\left(\right.$ Celexa $\left.{ }^{\circledR}\right)$, and escitalopram (Lexapro $\left.\AA\right)$. There are only diminutive differences among them, manifested mainly in the side-effect profile, half-life, and drug interactions associated with each.

The side-effect profile culled from clinical trials as well as real-world experience is rather favorable compared to TCAs. Great improvements were made with the lack of anticholinergic effects; however, somewhat bothersome reactions still remain. The majority of side-effects seem to appear and subside within the first few weeks of therapy. The most commonly reported adverse reactions with fluoxetine are nausea $(23 \%)$, headache $(21 \%)$, insomnia $(20 \%)$, anxiety $(13 \%)$, nervousness $(13 \%)$, and somnolence $(13 \%)$. Sertraline is likely to cause more nausea (27\%), while paroxetine is more likely to be associated with lethargy (24\%) than other SSRIs as reported in trials and clinical practice (PDR, 2002).

Sexual dysfunction is another effect common to all SSRIs. Unlike the other side-effects of headache, nausea, and dizziness that tend to dissipate within the first few weeks, sexual dysfunction can occur and persist late into therapy (Stokes and Holtz, 1997). For patients intolerant to this effect, other effective alternatives exist such as the heterocyclic antidepressant buproprion (Wellbutrin), which may actually increase sexual drive. 
Another salient difference among the SSRIs is the elimination half-life. Fluoxetine possess the longest half-life four to six days with chronic administration relative to all other antidepressants (Stokes and Holtz, 1997). This enables patients to have a convenient once-a-day or even every other day dosing schedule. In February 2001, a once-a-week formulation of Prozac was approved for patients already maintained on a stable dose of fluoxetine.

The titration that is necessary with many of the TCAs is not a factor for patients beginning therapy with fluoxetine. Consequently, patients can remain on the initial therapy dose throughout the treatment phases. However, because of this, if discontinuation is warranted, the wash-out phase for switching antidepressants is recommended to be much longer (four to five weeks) for fluoxetine than the other SSRIs. However, this recommendation is rarely followed in clinical practice.

Another class of compounds, similar in action to the SSRIs, exists as a treatment option for major depression. Combining the inhibition of serotonin with the inhibition of norepinephrine, a class of drugs known as SNRIs (serotonin-norepinephrine reuptake inhibitors) were produced. Venlafaxine (Effexor) and nefazodone (Serzone) are two compounds currently available. Significant efficacy over placebo has been documented for both drugs (Moller and Voltz, 1996; Rickels, 1994). 
Side-effects for these drugs are also more tolerable compared to TCAs. The most common adverse effects for venlafaxine are nausea, somnolence, dry mouth, dizziness, and constipation. Also, it is associated with blood pressure elevation. This phenomenon occurs in about 3\% of patients (Moller and Voltz, 1996). Nefazodone has similar sideeffects with less initial anxiety and sexual dysfunction than SSRIs, especially fluoxetine (Revicki et al., 1997).

\section{COMPARATIVE EFFICACY-FLUOXETINE}

In examining the comparative efficacy of fluoxetine and other antidepressants, relatively short-term studies reveal fluoxetine to be equally efficacious and distinctly more tolerable than TCAs (Nielsen et al., 1993; Workman and Short, 1993). These studies also support the claim that fluoxetine use is associated with lower rates of discontinuation than the TCAs.

Studies comparing fluoxetine with the other SSRIs in addition to other agents such as the SNRIs are also abundant. Double-blinded multicenter trials have demonstrated similar efficacy and comparable side-effects profiles for all of the SSRIs and SNRIs. Moderately to severely depressed outpatients assessed by HAMD improved similarly with fluoxetine or sertraline with at least a 55\% responder rate in each group (Aguglia et al., 1993). The occurrence of adverse reactions also was reported at similar rates, but the sertraline group had fewer drop-outs due to side-effects. This result may be due to the pre-treatment differences between groups that were observed. 
No significant differences were reported in a study of outpatients scoring at least 18 on the HAMD randomized to receive either fluoxetine (20 to $60 \mathrm{mg}$ daily) or paroxetine (20 to $40 \mathrm{mg}$ daily) (Gagiano, 1993). More than $60 \%$ of patients had a $50 \%$ reduction in HAMD scores in both groups. CGI scores also confirmed a greater than $60 \%$ response rate. Additionally, treatments were well tolerated with only three withdraws in each group out of 90 due to adverse effects.

A double-blinded study comparing fluoxetine to fluvoxamine assessed 93 outpatients with major depression (Rapaport et al., 1996). According to HAMD scores, no significant differences were found in treatment response after seven weeks of therapy. Both groups reported mild side-effects and only two patients in each group withdrew because of adverse events.

Comparisons of fluoxetine to citalopram have also been conducted (Patris, 1996). A double-blinded multi-center trial evaluating 314 depressed outpatients found no significant differences in efficacy or tolerability. Response was assessed according to a 50\% reduction in baseline MADRS scores. Fluoxetine-treated patients had a 76\% response rate, and citalopram-treated patients had a 78\% response rate. CGI results yielded over $80 \%$ of both groups reporting "much improved" or "very much improved". Ten patients withdrew from citalopram treatment and four from fluoxetine treatment.

A 12 week double-blinded randomized control trial compared fluoxetine with venlafaxine (Tylee and Bowden and Reynolds, 1996). Three hundred forty-one depressed outpatients 
were assessed by MADRS, HAMD, and CGI scores. Sixty-eight percent of fluoxetine patients and $63 \%$ of venlafaxine-treated patients responded to therapy according to HAMD scores. Similar results were obtained by the other scale scores with no significant differences detected between treatment groups. Similar results were reported in a study comparing fluoxetine to nefazodone (Rioux et al., 1996).

A recent publication in the Journal of the American Medical Association reported that there were no differences in efficacy of three leading SSRIs. (Kroenke, 2001). A total of 573 adults diagnosed with depression by their primary care physicians were randomized to receive either fluoxetine, sertraline, or paroxetine and were followed for 9 months. The primary outcome measure was change in the Medical Outcomes 36-item Short-Form Health Survey (SF-36) Mental Component Summary score. There were no statistical differences among the three treatment groups over the nine-month period in terms of effectiveness, discontinuation, or adverse effects.

There is clear consensus that fluoxetine is effective for the treatment of major depressive disorders. However, there is not a clear consensus on how effective it is. A metaanalysis of 26 trials testing fluoxetine in adult outpatients reported a mean response rate of 46.6\% (Depression Guideline Panel, 1993). While another study reported an average response rate of $64.6 \%$ for outpatients treated with fluoxetine (Einarson, 1995). Another more recent analysis comparing the newer agents against each other, including fluoxetine reported no significant differences with an average response rate of 53\% (Williams, et al., 
2000). However, most of these patients were recruited from inpatient settings and therefore, probably more severely ill.

As previously mentioned, scores obtained by HAMD as well as other scales are traditionally accepted as a measure of severity of depression. The aforementioned studies mainly evaluated moderate to severely depressed patients as indicated by the initial HAMD score. Documentation of efficacy for patients with more mild forms of the disorder is also important.

In 1993, a study was published addressing the issue of response to fluoxetine based on severity of depression (Pande and Sayler, 1993). The authors pooled results of 19 double-blinded randomized clinical trials involving the comparison of fluoxetine with either placebo or a TCA (amitriptyline, imipramine, desipramine, doxepin, or nortriptyline) to yield a total of 3,183 patients with major depressive disorder.

Patients were stratified into three severity levels (mild, moderate, and severe depression) based on total HAMD scores at baseline. The results were then re-analyzed to yield response and remission rates for each stratum. No significant differences in efficacy were found between groups based on severity of illness.

In all groups, fluoxetine was more effective than placebo with higher remission rates and as effective as TCAs with similar remission rates. However, remission rates were highest in the mild depression subgroup and lowest in the severe depression subgroup, as would 
be expected. The average response rate for patients with mild to moderate depression was $66.5 \%$ for the fluoxetine group. This study successfully illustrated the effectiveness of fluoxetine for all severity levels of MDD.

A review of newer antidepressant therapies including fluoxetine used to treat non-major depression or dysthymia corroborates this. Patients randomly assigned to receive an SSRI had a response rate of 59\% compared to $37 \%$ for the placebo group. All patients were treated in an outpatient setting. Even in mild to moderate depression, fluoxetine, as well as the other antidepressants, was demonstrated to be effective (Williams et al, 2000).

Data thus far regarding the use of fluoxetine to treat depressive disorders has seemingly justified its popularity among practitioners, making it a good representative for all SSRIs. Patients as well as practitioners have reaped the benefits of increased tolerability, equal efficacy, and convenient dosing compared to other antidepressants. The addition of the drug to the previously available arsenal of agents has made substantial strides in management of mental illnesses in a number of areas including the treatment of mild to moderate depression.

However, another therapy does exist that has been used extensively in Germany. Falling under a more unconventional approach, this herb has received much attention in the United States over the past few years. The herbal remedy being referred to is Hypericum perforatum, better known as St. John's Wort. 


\section{HERBAL THERAPY FOR MAJOR DEPRESSION}

St. John's Wort is a flowering plant that contains at least ten constituents or groups of components that may contribute to its pharmacological effects. The two primary classes of constituents are naphthodianthrones (hypericin and pseudohypericin) and flavonoids (hyperforin and adhyperforin). Most researchers have focused their attention on the effects of hypericin and hyperforin as possessing antidepressant effects. However, there are numerous compounds with documented biological activity.

Activity and potency may vary depending on the method of extraction and the percentage of standardized constituents. Most commercially available products are standardized based on a hypericin content of $0.3 \%$. The USP recommends that all preparations are standardized to no less than $0.2 \%$ hypericin/pseudohypericin or no less than $3 \%$ hyperforin based on analysis by high performance liquid chromatography (USP, 1999). Additionally, a specific extraction method has been identified that offers a high yield of hypericins and hyperforins (Poutaraud, 2001). Given the contribution of these critical compounds to the overall effectiveness of the extract, it is imperative that strict controls are employed during manufacturing.

The exact antidepressant mechanism of action is unclear at present. However, studies have demonstrated the extract to have multiple mechanisms of action in vitro. Once thought to be a monoamine oxidase (MAO) inhibitor, it has now been shown to have very little affinity for these receptors. Hydroethanolic extracts exhibit activity at gammaaminobutyric acid (GABA) receptors but only weakly inhibit MAO. Hydromethanolic 
extracts act similarly to reserpine by releasing stored seratonin (5HT) thereby increasing cytoplasmic concentrations causing reuptake inhibition in the synapse. These extracts also inhibit norepinephrine (NE) and dopamine (DA) (Muller, 1997).

Hypericin alone has been shown to inhibit the metabolism of DA. It has affinity at GABA type A and 5HT receptors but no significant affinity at adenosine, adrenergic, central benzodiazepine, or opioid receptors. Relatively low affinity was shown for Nmethyl-D-aspartate and MAO type A receptors. Hyperforin also inhibits neuronal uptake of DA, NE, 5HT, and GABA, whereas MAO inhibition is insignificant (Bilia, 2002). The combined effects on the various neurotransmitters most likely explain its clinical activity, rather than one simple mechanism.

Although a multitude of reports and studies suggest that the extract is well tolerated and generally safe to use, there is evidence that many of the herb's constituents interact with other drugs, mainly those metabolized by the hepatic Cytochrome P450 enzyme system. St. John's Wort is a known inducer of many of the isoenzymes such as $3 \mathrm{~A} 4,2 \mathrm{C} 9$, and 2D6 as well as P-glycoprotein expression (Ernst, 1999). Consequently, it can interact with a number of different drugs. Most reports involve decreases in plasma levels of the drugs, thereby reducing therapeutic effectiveness. Interactions can be quite severe such as with cyclosporine. Acute rejection of solid organ transplants have been reported (Turton-Weeks et. al., 2001). It is therefore recommended to avoid concomitant use of the herb and any drug affected by these isoenzymes, such as anticoagulants (warfarin), antiretrovirals, and oral contraceptives. 
Though not quite as extensive as its prescription counterpart, the research published on St. John's Wort is evident. In the early 1990's, European studies supplied most of the information regarding efficacy, tolerability, and safety of certain extracts for the treatment of mild to moderate depression. Today, published US trials on St. John's Wort document its use in the treatment of depression as well as continued anecdotal support.

In $A B C$ 's investigative report, medical editor, Dr. Timothy Johnson interviews Dr. Harold Bloomfield. Dr. Bloomfield is a Yale-educated psychiatrist and author of the best-seller "How To Heal Depression". He became interested in the herb's potential as an antidepressant and started reading and studying its effects before ultimately using it in clinical practice. The results he has seen with his patients prompted him to write another book titled "Hypericum and Depression" where he praises the ability of this herb to effectively treat mild to moderate depression with mild or no side-effects (Bloomfield and McWilliams, 1996).

So impressed with the results he has seen from the hundreds of patients for whom he prescribed the herb, Dr. Bloomfield emphasizes the importance of St. John's Wort by stating in his book and on national television that it should be the drug of choice for mild to moderately severe depression. He predicts that it will soon outsell all other antidepressants and become the leading therapy choice despite the massive marketing campaigns of pharmaceutical companies. That will be no small feat considering fluoxetine's sales of $\$ 2$ billion per year in 1995 alone (Murray, 1995). 
Many other experts in the field seem to share his enthusiasm. A psychologist at the National Institute for Health, Jane Steinberg agrees that there is good evidence of the herb's effectiveness (Benedict, 1998). Author of "St. John's Wort: Nature's Blues Buster", Dr. Hyla Cass has switched many of her patients from synthetic antidepressants to the herb and states that it is "unquestionably superior" to conventional therapy in the treatment of mild to moderate depression (Colchamiro, 1998).

Countries well versed in using phytomedicinals have provided excellent documentation and a wealth of information on clinical experience. Over thirty controlled studies have been conducted on Hypericum to demonstrate its effect in patients with depressive disorders. The British Medical Journal published a meta-analysis of 23 randomized clinical trials in which the authors found sufficient evidence to claim Hypericum's superiority over placebo in the treatment of mild to moderate depressive disorders (Linde et al., 1996). Dr. Cynthia Mulrow, one of the co-authors of the analysis, now recommends the herb as an alternative to her patients diagnosed with depression (Huff, 1998). The extract has been studied in over 1,700 individuals with most trials using the German extract LI 160, although several other brands have also been tested.

In a placebo-controlled, double blind study conducted by Harrer and Sommer (1994), 105 mildly depressed patients were given $300 \mathrm{mg}$ of the extract LI 160 three times a day for four weeks. Using the HAMD scale, treatment was assessed at baseline, after two and four weeks. Significant differences were recorded at two and four weeks of therapy with 
a $67 \%$ response rate for the treated group compared to a $28 \%$ response rate in the placebo group at week four $(\mathrm{p}<0.05)$. Improvements in depressive mood, psychological anxiety, and difficulty initiating sleep were also noted. Frequency of adverse drug reactions were not reported nor was an intent-to-treat analysis, a method of including all randomized subject's data into the final analysis, regardless of whether or not they completed the trial according to protocol. It is used to accurately represent the statistical magnitude of benefit.

Another placebo-controlled trial, 72 patients classified as having major depression were randomized to either a control group or treatment with $300 \mathrm{mg}$ of standardized extract three times a day (Hansgen, 1994). The HAMD and CGI were used to evaluate depressive symptoms and overall efficacy. Again, significant improvements were noted after four weeks. The response rate for patients taking the extract was $81 \%$ and $26 \%$ for those on placebo $(\mathrm{p}<0.001)$. Two patients in the placebo group and one in the active group reported adverse drug reactions.

Hypericum extract LI 160 (Jarsin-300) was studied in 39 patients suffering from depressive and psycho-vegetative irritations (Hubner et al., 1993). They were treated for four weeks with either placebo or at the daily dose of $900 \mathrm{mg}$ (300 $\mathrm{mg}$ three times a day). Approximately $70 \%$ of patients in the treatment group were free of symptoms after four weeks as measured by HAMD scores, which were significantly lower than placebo. Additionally, no adverse drug reactions were reported for either group. 
Similarly promising results were reported on the same brand of extract, Jarsin (Halama, 1991). A four-week trial involving 50 patients with mild or neurotic depression were treated with either the extract or placebo. At the end of the treatment period, $60 \%$ of the active group reported a significant improvement of symptoms while there was no detectable change in the control group. Only one patient treated with the extract complained of adverse drug reactions.

Additionally, LI 160 or placebo was used to treat sixty-five mild to moderately depressed patients in a double-blinded randomized trial lasting six weeks (Schmidt, 1993). Once again results favored active treatment significantly. Sixty-seven percent of patients responded to the extract. The response rate for the placebo group was $27 \%$. Fatigue and itching were reported side effects of the active treatment.

In a multi-center trial, 116 patients were randomized to treatment with liquid extract or placebo for six weeks (Harrer, 1991). Patients suffered from neurotic or reactive depression and were assessed according to the HAMD. Thirty-eight percent of patients treated with the Hypericum extract showed a reduction in depressive symptoms while $15 \%$ of patients on placebo improved. Two cases of adverse drug reactions were reported for the Hypericum group and nine for the placebo group.

Quandt's study (1993) consisting of 88 patients suffering from mild to moderate depression studied the efficacy of a liquid formulatin of the extract compared to placebo. The group treated with Psychotonin (liquid Hypericum extract, 30 drops three times a 
day) improved significantly. There were no reported adverse drug reactions for either group. Schlich and associates (1987) reported comparable results when they treated 49 depressive patients with the same extract.

Neuroplant, which is a dried extract of Hypericum, was used by Reh and Laux (1992) in this double-blinded controlled trial. Treatment continued for twice as long as most other published trials with assessments performed at eight weeks. HAMD scores were significantly lower, $70 \%$ decrease in baseline, in the active group compared to $45 \%$ decrease in the control group. Unfortunately, the study had very little power with a sample size of only 25 patients in the treatment group.

Attempting to address the issue of small sample sizes, this next trial enrolled 162 patients with mild to moderate depression (Schrader, 1998). Compared to previous trials, a relatively low dose of Hypericum was used—250 mg twice daily. However, similar results were obtained as previously documented. The responder rate for the treatment group was significantly greater than placebo, $56 \%$ versus $15 \%$ respectively $(\mathrm{p}<0.001)$.

The first large clinical trial conducted in the United States evaluated the efficacy of St. John's Wort $300 \mathrm{mg}$ given three times a day, increased to a maximum of $1200 \mathrm{mg}$ if necessary, compared to placebo in two hundred patients (Shelton, 2001). This eightweek trial conducted at 11 acedemic medical centers enrolled patients with moderate to severe depression and assessed rate of change in HAMD scores in addition to response rates as measured by HAMD or CGI scores. Neither change in baseline HAMD score 
nor response rates differed between groups. Even after stratifying the sample based on initial severity of depression (those with a mean HAMD score greater than 22 and those less than 22), there was no significant difference in rate of change in HAMD scores for either group.

This study was criticized for a number of reasons. The gold standard for testing inefficacy is a three-arm study comparing the new agent, placebo, and a reference drug with known efficacy (Hawley, 2001). Since there was no reference drug, it is hard to determine whether St. John's wort was truly ineffective or if the patients represented a more treatment-resistant group. Furthermore, the placebo response of $18.6 \%$ was notably lower than in most trials (Cott, 2001).

Another point raised was that the sub-group analysis of less severely ill patients only included those with scores of 20 and 21 on the HAMD. Many argue that these score do not adequately represent mild to moderate depression, and therefore, the lack of a difference does not confer lack of efficacy in this population (Fugh-Berman, 2001). The authors did clearly state this in their discussion.

The efficacy and tolerability of an extract, WS 5572 standardized to $5 \%$ hyperforin, was studied against placebo in 72 patients with mild to moderately severe (HAMD scores of at least 16 or more) depression (Kalb, 2001). The study was performed over a period of approximately six weeks (42 days) and focused primarily on efficacy and tolerability of the extract. Favorable results for WS 5572 were reported on all indicators as assessed by 
scales such as CGI and HAMD. Improvements could be detected as soon as day seven of therapy. At day 42 , response rate for the extract was $62 \%$ versus $43 \%$ for placebo. Adverse effects were mild for both groups including three cases $(8 \%)$ of upper respiratory symptoms for active treatment, which may have not been treatment related.

In reviewing more recent literature, another extract, WS 5570, standardized to 0.12 $0.28 \%$ hypericin and 3-6\% hyperforin, was studied in a randomized, double-blinded, placebo-controlled trial involving 375 outpatients (Lecrubier, 2002). The six-week study, which took place in several centers located throughout France, enrolled patients suffering from mild to moderate major depression as classified by the Diagnostic and Statistical Manual (DSM-IV) criteria. At the end of the six-week period, patients were assessed to determine the change in HAMD score from baseline as well as the melancholia subscore, total score on the MADRS, the 58-item version of the Symptom Check List (SCL-58), and the CGI.

Intent-to-treat analysis demonstrated a significant difference in mean change of HAMD from baseline for treatment group compared to placebo. Patients with a higher initial HAMD score (22 or more) experienced a larger treatment effect (greater magnitude in score changes) than those patients with lower initial scores, suggesting that St. John's Wort may be more effective for more severe forms of depression. Those responding to therapy (using the typical definition of at least a 50\% decrease in baseline score) were significantly greater for the extract $(52.7 \%)$ compared to placebo $(43.3 \%)(X=4.04, d f=1$, $\mathrm{p}<0.05$, two-sided). Additionally, $24.7 \%$ of patients experienced remission (score of six 
or less on the HAMD) with the extract compared to $15.9 \%$ of patients in the placebo group, which also represented a significant difference $(X=4.55, \mathrm{df}=1, \mathrm{p}=0.03$, two-sided $)$. All other secondary measures were positive for the extract compared to placebo.

This trial was one of the larger, well-designed trials conducted to date. It was published just four months after another pivotal trial that demonstrated a negative outcome for the herb. This trial is mentioned in a subsequent section, focusing on comparative therapy, as it included a reference drug (Hypericum Trial Depression Study Group, 2002).

Several meta-analyses have analyzed both placebo-controlled trials against the extract as well as those comparing standard antidepressant therapies. The inclusion criteria for each of these differ somewhat. For example, the first meta-analysis by Linde et al. included 13 trials that compared the extract to placebo. An overall response rate of $55 \%$ was reported for the Hypericum group while $22.3 \%$ was reported for placebo (Linde, 1996). Whereas, Kim et al. employed much more rigorous criteria for study inclusion and identified only two placebo-controlled studies that qualified. The pooled response rate for this analysis was $73.2 \%$ for Hypericum and $37.9 \%$ for placebo (Kim, 1999).

Although some studies possess stronger methodologies than others, overall the evidence purported in these double-blinded placebo controlled trials favors St. John's Wort in the treatment of mild to moderate depression. Anecdotal evidence available over the centuries of use in Germany adds strength to this argument of effectiveness. However, 
one must also consider the effectiveness of St. John's Wort relative to the prescription antidepressants before advocating its widespread use.

\section{COMPARATIVE EFFICACY-ST. JOHN'S WORT}

A number of studies have been conducted comparing Hypericum compounds with conventional antidepressants. Trials such as these lend themselves to making stronger arguments regarding the use of St. John's Wort since the efficacy of the comparator drugs have been well documented. A criticism of many of the trials is the use of a lower than standard dose of comparison drugs. One author reasons that chosen doses were on the lower end of the acceptable therapeutic range to facilitate blinding of patients (Vorbach, 1994). At higher doses, the classic side-effects of TCAs may be evident. In addition to this, one can argue that the doses of TCAs reported (e.g. imipramine $75 \mathrm{mg}$ ) are what most patients receive in the outpatient setting (Wheatley, 1997).

The multi-center trial mentioned above (Vorbach, 1994) compared imipramine $75 \mathrm{mg}$ daily to the brand extract, Jarsin (LI 160) $900 \mathrm{mg}$ daily in a randomized, double-blinded trial. Success after six weeks was evaluated on the HAMD, and CGI scales. Both groups significantly improved according to all three scales, but no inter-group difference was detected. Similar findings were reported in another study comparing imipramine with Psychotonin (Werth, 1989).

The extract LI 160 was again used to test efficacy against amitriptyline in this doubleblind, randomized trial (Wheatley, 1997). Patients with mild to moderate depression 
classified according to DSM-IV criteria and HAMD scores were followed for six weeks and treated with either $75 \mathrm{mg}$ daily dose of amitriptyline or $900 \mathrm{mg}$ extract daily. Results based on an intent-to-treat analysis revealed no significant difference in regards to response rate and CGI severity scores. There was a clear advantage in tolerability for the extract with $32 \%$ of extract patients withdrawing due to side-effects compared with $52 \%$ of amitriptyline patients.

Woelk (2000) used imipramine as the comparator drug in this trial testing 324 outpatients with mild to moderate depression. The dose used more closely approximated that recommended by most prescribers, titrated over seven days starting with $25 \mathrm{mg}$ twice daily and increasing to $75 \mathrm{mg}$ twice daily. The Hypericum extract ZE 117, standardized to $02 \%$ hypericin was tested for therapeutic equivalence to the standard therapy. No differences were documented between the two groups with respect to mean change in HAMD scores from baseline to the end of week six. Again, tolerability favored Hypericum with only $30 \%$ reporting adverse effects compared to $63 \%$ for impramine.

St. John's Wort has also been evaluated in patients with more severe depression. An ICD-10 code (severe episode of a major depressive disorder, recurrent, without psychotic symptoms) designated inclusion in one controlled six-week trial (Vorbach, 1997). Patients were randomized to $1800 \mathrm{mg}$ LI 160 extract or $150 \mathrm{mg}$ imipramine per day and assessed according to the HAMD and CGI. Similar responder rates (35.3\% for LI 160 and $41.2 \%$ for imipramine) and global assessments (61.2\% for LI 160 and $70 \%$ for imipramine) were found according to HAMD and CGI respectively. However, total 
HAMD scores and CGI "therapeutic effect" were not equivalent for the two groups with imipramine fairing better in both. Tolerability, once again, was greatly favored toward LI 160.

Meta-analyses of trials comparing St. John's Wort to standard antidepressants such as amitriptyline have also been published. As mentioned previously, two separate analyses yielded similar results (Linde, 1996 and Kim, 1999). Ten trials were identified in the first analysis and reported a response rate of $63.9 \%$ for Hypericum versus $58.5 \%$ for the other antidepressants. The second published meta-analysis of four trials involving standard antidepressant therapies compared to Hypericum demonstrated response rates of $66.4 \%$ and $64 \%$ respectively. Both analyses showed improved tolerability with the extract over the standard TCAs with fewer drop-outs (12.6\% vs. $16.2 \%)$ and less adverse effects reported.

The majority of clinical research performed on this herb has supported the notion that St. John's Wort may well have a place in clinical practice for treating mild to moderate depressive episodes. In order to better assess the possibility of it becoming the standard of care, additional studies have been conducted against the more commonly prescribed SSRIs. Three studies have examined the benefits of St. John's Wort in comparison to sertraline, and three have used fluoxetine as the comparator.

Brenner et al (2000) studied a small group of patients diagnosed with major depression based on DSM-IV and a score of at least 17 on the HAMD. A dose titration of $600 \mathrm{mg}$ of 
LI 160 or $50 \mathrm{mg}$ of sertraline was utilized for the first week of therapy at which time patients were increased to $900 \mathrm{mg}$ or $75 \mathrm{mg}$ respectively for the remaining six weeks. Response rates were comparable with no statistical differences between sertraline (40\%) or Hypericum (47\%). Change in HAMD scores also did not differ. A post-hoc analysis indicated adequate power of the study to detect treatment effect differences. Both treatments were well tolerated with only two reports of adverse effects in each group.

A comparison of these two agents was reported in another small trial conducted in Canada (van Gurp, 2002). Eighty-seven outpatients with major depression evidenced by a score no less than 16 on the HAMD were randomized to receive either St. John's Wort or sertraline for 12 weeks. Dose escalation was also allowed in this trial with 50 to 100 $\mathrm{mg}$ of sertraline used or $900 \mathrm{mg}$ to $1800 \mathrm{mg}$ of the extract.

Assessments were made based on the BDI, change in HAMD, as well as self-reports of side-effects and compliance at various stages throughout treatment. The primary outcome measured was change in HAMD, BDI, and difference in side-effects reported at 12 weeks. Intent-to-treat analysis failed to reveal any statistical differences in measurements of efficacy between the treatment groups. In addition, St. John's Wort was found to be better tolerated than sertraline.

The largest of all trials, jointly funded by the National Institute of Mental Health and the National Center for Complementary and Alternative Medicine, studied a total of 340 patients diagnosed with major depression as determined by DSM-IV criteria, a HAMD 
score of at least 20, and a maximum score of 60 on the Global Assessment of Fuctioning (Hypericum Trial Depression Study Group, 2002). Patients were randomized to receive either the extract LI 160 , standardized to $0.12 \%$ to $0.28 \%$ hypericin, placebo, or sertraline acutely for eight weeks, then an additional four months for those exhibiting a response. The primary efficacy end point was the mean change in HAMD scores at week eight. Full and partial response was also measured based on CGI scores of 1 or 2 and HAMD score of 8 or less or CGI of 1 or 2 and HAMD score of 9 to 12, respectively.

Notably, no differences in change in HAMD score or full response rate were detected among the three groups at week eight. However, sertraline was superior to both placebo and Hypericum on the CGI-I (improvement) score at week eight, though this was a secondary outcome measure. Adverse events were lower with Hypericum than sertraline, but neither had reports of serious events.

Although this trial failed to demonstrate any benefit of the extract over placebo, the fact that a well-known and previously evidence therapy for depression (sertraline) also failed to demonstrate efficacy over placebo must be taken into consideration. Additionally, this trial tested the extract in patients with more severe depression, most of whom had been diagnosed with depression for greater than one year (32\% greater than 2 years). Hence, these patients may in fact have been more treatment resistant than those tested in other trials. Though this study was methodologically sound, more questions were raised than answers elucidated. 
Seventy patients with ICD-10 codes indicating mild to moderate depression were randomized to receive either $150 \mathrm{mg}$ twice daily of Hypericum extract (Calmigen) or fluoxetine $20 \mathrm{mg}$ twice daily (Behnke, 2001). Rationale for these unconventional doses was not discussed. Equivalence was evaluated by comparing efficacy measured in terms of percentage reduction in HAMD scores. Therapeutic equivalence was inferred based on the Food and Drug Administration's recommendation stating that the study drug must be $80 \%$ to $125 \%$ as efficacious as the comparator drug.

The mean HAMD score decreased by $50 \%$ in the Calmigen group while the fluoxetine group showed a $58 \%$ reduction in mean score, each representing a statistically significant decrease $(\mathrm{p}<0.001)$. Between group analysis, however, failed to demonstrate a statistical difference in group mean scores $(\mathrm{p}=0.23)$. The overall response rate defined as $\geq 50 \%$ reduction in HAMD score was 55\% for the Calmigen group and $66 \%$ for fluoxetine $(\mathrm{p}=0.41)$. Hence, the extract was approximately $83 \%$ as efficacious as fluoxetine. The authors state this study demonstrated equivalence between Calmigen and fluoxetine based on the predetermined definition of equivalence. However, given the small sample size, the study may not have been powered adequately to detect a true difference.

An analysis of the literature on St. John's Wort and Prozac was compared to determine if response rates were similar among the various trials (Voltz and Laux, 2000). The authors included trials testing efficacy of either compound in patients identified as having mild to moderate depression based on ICD-10 codes or scores of $\leq 24$ on the HAMD. Absolute reduction and percentage reduction in HAMD scores were combined and compared for 
the two compounds. No difference in absolute or overall percentage reduction was found. However, sample sizes were not taken into consideration, and therefore, this was not a meta-analysis but rather a gross comparison of all studies. Due to the weakness of the methodology, clinically relevant inferences are difficult to make. The authors admit to this fact and state that these preliminary data suggest possible equivalence but rigorous trials are recommended.

The most compelling multi-centered trial to date compares the efficacy and tolerability of St. John's Wort extract Ze117 to fluoxetine (Schrader, 2000). A total of 240 outpatients with initial HAMD scores between 16 and 24 were randomized to either $20 \mathrm{mg}$ fluoxetine daily or $250 \mathrm{mg}$ Ze117 twice daily. The primary outcome measured was the change in mean HAMD score, measured at week zero and week six. Equivalence was defined prospectively as $\leq 3$ points difference in mean score at endpoint for the two treatments. Intent-to-treat analyses were conducted on all variables. The two treatments were determined to be equivalent based on the change in mean HAMD score of 7.25 (95\% Confidence Interval (CI): 8.1 to 6.4$)$ for the fluoxetine group and 8.11 (95\% CI: 9.0 to 7.3) for the extract group. Secondary variables measured were responder rate, compliance, mean CGI, and safety. There were no differences noted in compliance rates, change in CGI for items 2 or 3, or in patients' self-assessments. Significant differences were measured in responder rates ( $40 \%$ for fluoxetine and $60 \%$ for Hypericum extract, $\mathrm{p}=0.05$ ) and mean change in item 1 (level of improvement, with 1 being "very much improved" and 2 being "much improved") of the CGI (1.3 for fluoxetine and 1.6 for the extract, $\mathrm{p}=0.03)$. 


\section{PHARMACOECONOMIC STUDIES OF DEPRESSION}

Decision analysis is one particular method used to conduct cost-effectiveness analyses. Because of its unique ability to yield information under conditions of uncertainty, this approach has been taken by many researchers to evaluate costly, controversial, or questionable drug therapies. In the field of mental health substantial pharmacoeconomic research, specifically decision analyses, exists demonstrating the apparent demand for and use of economic modeling.

In one study, Revicki et al (1997) compare cost-effectiveness and cost-utility of three antidepressant therapies, nefazodone, imipramine, and fluoxetine. The study estimated lifetime medical costs of treatment of depression from a managed care perspective and calculated quality-adjusted life years (QALYs) as the measure of utility. The main goal of the study was to assess the efficiency of using newer antidepressants (nefazodone and fluoxetine) as first-line treatment. Each treatment was assessed individually. Subsequently, a step-wise approach of using imipramine as a first-line treatment then fluoxetine only when initial therapy failed was assessed. This allowed for an evaluation of the financial impact resulting from initial choice of initial antidepressant.

The model was based on the expert opinion of a panel of primary care physicians, psychiatrists, and a clinical pharmacologist in addition to information obtained from the medical literature. Pathways in the model were based on ideal primary care practices. 
Probabilities between depression and remission were based on literature and the panel members.

A salient feature of the model was compliance states. One assumption was that patients would be in full, partial, or a non-compliant state and were able to transition between compliance states over the course of future depressive episodes. The panel assumed a greater percentage of patients would be compliant with fluoxetine and nefazodone due to dosing and tolerability compared to imipramine.

For imipramine, $30 \%$ were assumed to discontinue treatment within the first six weeks. Of the remainder, $40 \%$ would be fully compliant, $30 \%$ partially and $30 \%$ minimally compliant. Nefazodone and fluoxetine were assumed to have the same compliance rates since reports in the literature suggest higher compliance rates with newer antidepressants compared to TCAs, and also there is little difference between once a day dosing and twice a day dosing regarding compliance (Blackwell, 1979). Resultant rates for the two were: $20 \%$ discontinue treatment after six weeks, and of the remaining, $50 \%$ fully, $30 \%$ partially, and $20 \%$ minimally compliant.

QALYs were calculated by using standard gamble technique to estimate the utility of taking one of the three drugs while remaining in remission then multiplying the estimated life-expectancy for each treatment regimen. One year on nefazodone was rated at 0.87 , imipramine was 0.80 , and fluoxetine was 0.86 on a utility scale of 0.0 to 1.0 . 
Once the total costs for each treatment was determined, it was then divided by the QALY for that treatment to yield cost-effectiveness ratios. Imipramine had the lowest life-time medical cost, but the lowest QALYs. QALYs were greatest for nefazodone. When ratios were compared, it became evident that nefazodone was the most cost-effective (lowest cost per QALY) treatment approach.

A sensitivity analysis, substituting a range of values for point estimates that are not certain, was performed on compliance rates, recurrence rates, and the discount rate. All analyses were one-way except for the last, which varied both compliance and recurrence rates. No substantial differences were noticed even when the discount or recurrence rates were changed. Compliance had the biggest impact, but the newer antidepressants were still found cost-effective even with equal compliance rates among all treatments.

Another study demonstrating the use of decision analysis used a decision tree to evaluate oral antidepressant therapy in the management of major depressive disorders (Einarson et al., 1995). The introduction of a new antidepressant, venlafaxine, spurred the interest in researchers to conduct this study. Two models of clinical practice were developed to track in- and outpatient management of major depressive disorders for approximately one year. The comparator agents were SSRIs (fluoxetine and paroxetine), SNRI (venlafaxine), heterocyclic (trazodone and maprotiline), and tricyclic (amitriptyline and imipramine) antidepressants. To determine overall cost-effectiveness, the outcomes measured were total direct medical costs and symptom free days. 
An expert panel was utilized to determined the medical management of patients with major depression. Medical care such as lab tests, physician visits, and hospitalization as well as amount and duration of drug were estimated for each treatment alternative. Averaging the reimbursement rates of three HMOs' derived costs for medical treatment. Drug costs were estimated by the acquisition cost published in the 1993 edition of the Red Book multiplied by the number of tablets per day for each treatment regimen.

Data on success rates of each treatment were obtained through a separate meta-analysis performed by the authors. SSRIs had the highest estimated outpatient success rate (fluoxetine, 64.6\%) followed by heterocyclic antidepressants (HCAs) (trazodone, 58.9\%). Venlafaxine and amitriptyline had the highest rate of response for inpatients $(62.4 \%$ and $50.9 \%$ respectively).

The outpatient model followed patients through the course of treatment for one year. Initial response to therapy was assessed at four weeks. Those failing to respond were treated with a different drug (all drugs were backed up by a TCA, TCAs were backed up by SSRIs) and reassessed at four weeks. Those responding were reassessed at six months with all failures following the same pathway for initial failure, and all successes were determined to be in remission for the remainder of time. The inpatient model was the same except for a 14-day hospitalization period built in for those failing to respond to therapy. 
Symptom free days were estimated by assuming that days following successful treatment were symptom free since the patient would not be a burden on the health care system. In this sense, a symptom free day is the same as a successfully treated patient. Therefore, the results of the ratio comparing costs to effectiveness measured by clinical success should yield the same result as the ratio comparing costs to effectiveness measured by symptom free days. To no surprise, the most cost-effective agent was the same regardless of whether symptom free days or clinical success was the outcome. The lowest cost-effectiveness ratio in the inpatient model was obtained by the treatment regimen employing venlafaxine as the initial therapy. For outpatients, HCAs were the most cost-effective treatment regimen followed by SSRIs.

Sensitivity analysis was performed on drug and medical costs and clinical success rates to determine how robust the results of the base case analysis were. Because more than two treatments were being evaluated, a rank order stability analysis was used to confirm the base case results by yielding elasticity values. Elasticity values greater than one indicate that the key variables (e.g. drug or medical costs) factored into the statistical basis of relative cost-effectiveness ratios, when varied, will compromise the rank order and undermine stability. All elasticities were less than one, confirming the initial results obtained.

The prevalent use of economic modeling is global. The Canadian Journal of Psychiatry published an article on the direct cost of treatment of depression with paroxetine compared to imipramine (Lapierre, 1995). A computer-simulated decision tree was 
developed to model treatment patterns of depression over a twelve-month period. Effectiveness data were obtained from the literature as well as a physician panel. Costs associated with medical management and pharmacotherapy were obtained from the Ontario Ministry of Health and the Ontario Drug Benefit Formula respectively.

The model developed follows patients through a typical clinical pathway in which initial treatment prescribed is either continued or discontinued due to failure to respond. Patients continuing treatment either complete a course of treatment for six weeks or relapse. Those continuing treatment are reassessed at six months and either end therapy or relapse. All relapses are followed by a second six-month course of treatment with the same drug. Initial failure on treatment is followed by changing the drug, hospitalization, electroconvulsive therapy, or visiting another physician and repeating the pathway stated above. Probabilities associated with each clinical pathway were estimated by the physician panel.

Data for the modeling of continuation, dropout, and relapse rates were obtained from three separate double-blinded randomized controlled trials. The first trial compared paroxetine to imipramine and reported continuation (response/tolerance) rates of 57.5\% for paroxetine and $46.4 \%$ for imipramine. The second study compared paroxetine to placebo and reported a relapse rate of 5\% at six months for paroxetine. A separate longterm study of six months duration comparing imipramine to placebo was used to obtain a relapse rate of $20 \%$ for imipramine. To remain conservative, the model used $10 \%$ relapse rate for each treatment. 
Once the base case variables were entered into the model and cost-effectiveness ratios were calculated, sensitivity analyses were performed. The following variables were evaluated over a given range: price of paroxetine, cost of hospitalization, relapse rate for both drugs, and the continuation rate for paroxetine. One-way analysis was performed on each.

It was determined that management of major depression with paroxetine produced a lower annual direct cost than that of imipramine. Despite the ten-fold higher acquisition cost of paroxetine, it yielded a lower cost of treatment failure, which accounted for a majority of the total direct cost of treatment. Cost of hospitalization was the only variable that affected the outcome of the analysis, as demonstrated in the sensitivity analysis. As the cost of hospitalization decreased, imipramine became more costeffective. A two- or three-way sensitivity analysis may have resulted in more variables impacting the final result.

The same treatment alternatives, paroxetine and imipramine, were evaluated in an earlier study published by Jonsson and Bebbington (1994). They also used a decision analytic model to evaluate cost-effectiveness of treatment for major depression. A third party perspective was taken to assess costs over a period of twelve months.

A panel of experts, psychiatrists, general practitioners, and hospital pharmacists, developed the possible clinical pathways in the model. Initial choice of treatment was 
either successful or the patient discontinued (measured by dropout rate) therapy. The probability of drop out for each treatment was based on a pooled analysis of six randomized double-blinded placebo-controlled trials that lasted six weeks. The rate of dropouts for paroxetine was $42 \%$ and $56 \%$ for imipramine. In the model, all dropouts switched drug therapy so one full course of each treatment (paroxetine and imipramine) was included in the cost of treatment failure.

If a patient did not drop out, they were considered a treatment success. The possibility of relapse was included for all successfully treated patients. Those relapsing were treated with a second course of therapy and reassessed. Again, patients were either successful or dropped out of therapy after relapse treatment. The relapse rate was established by panel members as $25 \%$ for each treatment.

Expected costs for each pathway in the model were calculate by multiplying the cost associated with the outcome of each pathway by the probability of that outcome occurring. The cost-effectiveness of each treatment was calculated as cost per successfully treated patient. Paroxetine had a lower expected cost than imipramine therapy and ended up being more cost-effective than imipramine.

The results proved to be very stable when sensitivity analyses were conducted. All mean values in the model were substituted with the extreme values included in the range given to each variable. The variables that are mentioned in the article as being varied over the 
lowest and highest possible values were cost of hospitalization, effectiveness of treatment, and cost of treatment failure.

Hospitalization costs had no baring on the results since this represented such a small fraction of overall cost of treatment. Cost of treatment failure however, did prove to be rather sensitive though did not change the outcome. As the cost of treatment failure decreased, the expected cost of paroxetine drastically decreased. However, the expected cost of imipramine remained relatively unchanged. Both the highest and lowest values of treatment failure resulted in paroxetine having the lower cost-effectiveness ratio. For the variable success, as the rate of success increased for paroxetine and remained constant for imipramine, paroxetine became more cost-effective until the point of dominance, where the treatment offers better effectiveness at a lower cost. At the lowest end of success ( $50 \%$ for paroxetine), imipramine had a lower expected cost but a higher costeffective ratio.

A recent review of pharmacoeconomic studies of SSRIs included the four studies discussed above in addition to several others that used decision models to assess costeffectiveness (Frank, 2001). The Canadian Coordinating Office for Health technology Assessment (CCOHTA) assessed cost per QALY of three treatment scenarios, initial treatment with an SSRI followed by TCA, TCA followed by an SSRI, or TCA therapy alone (Baladi, 1997). The model followed treatment over a period of 9 months and outcomes were analyzed based on a third party payer perspective. 
The parameter for success was continuation on treatment without relapse or therapy disruption. Data from a previous trial was used to determine QALYs, whereas probabilities and costs were derived from meta-analysis from randomized clinical trials plus expert opinion and claims data from Quebec, respectively. Direct medical costs were highest with TCA alone (\$1779). The SSRI to TCA strategy was $\$ 1334$ compared \$1214 for the TCA to SSRI strategy. However, the treatment strategy utilizing an SSRI as initial treatment with subsequent treatment failures switching to TCAs resulted in the lowest cost per QALY and therefore the most cost-effective alternative.

\section{CONCLUSION}

What has been learned thus far is that since the introduction of TCAs, there has not been any tremendous improvement regarding efficacy of antidepressants. However, the safety and adverse effect profiles of drugs such as fluoxetine have made significant advances toward improved compliance, tolerability and dosing. To date, a number of studies have been conducted comparing the TCAs and heterocyclic compounds to newer therapies such as the SSRIs and SNRIs. Since these compounds have made such vast improvements in therapy regarding safety and side-effects but carry with them high price tags, cost-effective analyses were warranted.

Their relatively expensive price may actually be commensurate with these enhanced features if utilization of health care services is decreased with their use. Reviewing the literature, it is apparent that decision analysis modeling is a popular method of evaluating drug therapy. Many cost-effective analyses have sided in favor of the newer drugs such 
as paroxetine over the less costly TCAs or HCAs specifically for this reason-- a decrease in physician visits and hospitalizations, which in turn drastically lowers total costs of care.

By the same token, any new antidepressant therapy on the market possessing improved qualities needs to be evaluated based on the best alternative currently being utilized. Only then will the results of the study possess external validity (Luce and Simpson, 1995). St. John's Wort has been evaluated in patients with mild to moderate depression and in one study testing its use in severe depression. Comparisons of efficacy have been directed toward standard TCAs such as imipramine and amitriptyline and against placebo. Also, open label studies following patients in a clinic setting have evaluated effectiveness.

All of these studies have taken a clinical perspective, assessing side-effects, safety, and efficacy. Though use of modeling to evaluate cost-effectiveness of antidepressants has been demonstrated to be widely used and accepted, there are no studies to date that examine St. John's Wort vis-à-vis fluoxetine, the two therapies proposed in this project. In fact, no evaluation of clinical parameters in combination with its economic impact has been done.

To get a better picture of where this extract fits in the treatment algorithm for mild to moderate depression, a cost-effective analysis is warranted. The previous data seem to point to the conclusion that St. John's Wort may be just as effective as prescription 
antidepressant therapies in the treatment of mild to moderate depression. However, the question still remains whether or not St. John's Wort is more cost-effective than prescription alternatives.

In the absence of any published information regarding relative cost-effectiveness, it is wise to compare St. John's Wort to one of the most commonly prescribed antidepressants in the outpatient setting. Fluoxetine, given its frequent use and now generic availability, shall serve as a representative of the class of SSRIs to which alternative treatments shall be compared. This study shall serve as a model comparison of the extract against fluoxetine comparing clinical and economic parameters using the method of decision analysis. 


\section{CHAPTER 3}

\section{METHODS}

\section{INTRODUCTION}

The purpose of this study is to evaluate the relative cost-effectiveness of St. John's Wort compared to fluoxetine based on a third party perspective for the treatment of mild to moderate depression. The majority of third party payers restrict coverage of pharmacotherapy to prescription status drugs only. St. John's Wort is currently an overthe-counter herbal supplement. It is noteworthy however to consider the effect of increasing the scope of coverage to include St. John's Wort for the treatment of major depressive disorders (MDD) including mild to moderate depression, if the therapy proves to be a more cost-effective alternative.

To maximize external validity, St. John's Wort is compared to the current standard of therapy as determined by a panel of experts with experience in treating this disorder. Fluoxetine, the comparator drug, is used as a representative of the class of drugs known as SSRIs (selective seratonin re-uptake inhibitors). This class of antidepressants are the most often used for the treatment of MDD (Sclar, 1998).

The main goal of the study is to assess the cost-effectiveness of St. John's Wort relative to that of fluoxetine when used to treat patients diagnosed with mild to moderate depression managed entirely in an outpatient setting. Due to the exclusion of patients with more severe debilitation, it was felt that these patients would not require hospitalization. 
Additionally, it was felt that this exclusion would better represent real-world costs given the chronic nature of the disease commonly being managed in primary care clinics. In order to attain this goal, the following objectives shall be met:

Objective A. Calculate the expected cost associated with the treatment of mild to moderate depression using fluoxetine at the initial dose of $20 \mathrm{mg}$ once a day.

Objective B. Calculate the expected cost associated with the treatment of mild to moderate depression using St. John's Wort at the initial dose of $300 \mathrm{mg}$ three times a day.

Objective C. Assess the overall cost per successfully treated patient (cost-effectiveness) of St. John's Wort.

Objective D. Assess the overall cost per successfully treated patient (cost-effectiveness) of fluoxetine.

Objective E. Assess the incremental cost-effectiveness of St. John's Wort compared to fluoxetine.

Objective F. Assess the robustness of the findings by performing sensitivity analyses. 
Identification of the research question, the alternatives, and the objectives of the study establishes the conceptual framework for cost-effectiveness analyses (Bootman, 1996). In order to expand upon this framework and achieve the stated objectives, a decision analysis model was created to capture the production relationship involved in the treatment of depression in the clinical outpatient setting. The production relationship identifies and quantifies outcomes (health and resource) for each alternative therapy and provides the technical framework for the assessment of cost-effectiveness for both drugs.

Several advantages exist in utilizing the decision analytic approach to model the treatment of depression. The flexibility of models allow for varying durations of treatment, numerous treatment options to be compared, and the clinical scenario can be based on real-life practice as opposed to controlled settings of randomized controlled trials. Models typically extrapolate data beyond that which has been studied. Also, they can provide information relative to decision-makers in a timely fashion.

\section{DECISION ANALYSIS}

Decision analysis is a method of breaking down a problem or question into stepwise outcomes and assigning a utility or cost to each outcome. The delineation of outcomes can be represented in a structure called a decision tree, the tool used in this study to calculate the cost-effectiveness ratios. The tree enables visualization of different choices and chances represented in a clinical scenario (Lawler, 1995). 
Within the structure of the decision tree, there are two forms of nodes, a probability or chance node and a decision node. The decision node represents a place in the tree where a decision or choice must be made (the initial question at hand). In this model, the decision node is followed by the treatment choices St. John's Wort and fluoxetine. A chance node occurs after a decision has been made and two or more outcomes follow. Each of these outcomes has a probability of occurring, which is represented by the value or definition given to the chance node and must equal 1.0 when the branches of that node are summed. The branches of the tree expand until all relevant outcomes have been identified and a final outcome is reached, which is called the terminal or payoff node (Lawler, 1995).

The number of outcomes or branches of the tree can be very extensive and depends to some extent on the scope of the analysis. Based on the scenario being modeled, a time frame must be defined. Determining the length of time for which a decision is modeled helps to establish the extent of the decision tree.

Once the structure of the tree is drawn with all relevant outcomes included, the next step is to assign probability estimates and costs and/or utilities associated with each outcome. Ultimately, probability estimates are based on clinical studies with good methodological quality that represent well the population being studied. Unfortunately, data are not always available even in the vast medical literature that is published. In those instances, it is necessary to rely on expert opinion. It is important to remember that the stronger the 
evidence used in the model, the more robust the inference one can make from the results of the model (Richardson and Detsky, 1995).

The next estimates to be entered into the tree are the costs associated with each outcome. Criteria used to establish costs depend on the chosen perspective of the study. Following the incorporation of all relevant data, the model is then analyzed. The process employed in the calculation of cost-effectiveness is called "folding back" the tree. In this process, probability estimates for each outcome are multiplied by the cost of the outcome and these products are then added together. It begins at the terminal node and folds back toward the root (decision) node until an expected value is calculated for each decision. The best decision based on the assumptions and criteria defined (lowest cost per highest benefit) can then be determined.

As stated previously, some of the data programmed in the tree are not certain but are best guess estimates or underlying assumptions. It is of particular interest to test these estimates over a range of values to see how the final decision is affected. This process is called sensitivity analysis (Tom and Schulman, 1997).

Each uncertain variable is defined by a plausible range of values on which the analysis will be based. Varying one variable at a time is called one-way sensitivity analysis. Changing the value of a number of different variables at the same time leads to multi-way sensitivity analysis. In executing this type of analysis, the robustness of the data is tested; 
it can be determined to what extent each variable or assumption is a predictor of the final outcome.

The decision tree model for this study was developed using the software Decision Analysis by TreeAge (DATA 3.0). This software program allows for the use of variables to define or represent probability and payoff values. Using this method allows for the calculation of base case (best guess) results and later changing those estimates that are uncertain or thought to be important predictors of the final result to yield best and worse case scenarios. DATA is similar to other decision analytic software such as SMLTREE (James Hollenberg, 526/625-4332) and Supertree (Strategic Decisions Group, Menlo Park, CA.).

DATA calculates cost-effectiveness ratios by first deriving the expected cost at each chance node then the expected effectiveness and subsequently divides expected cost by expected effectiveness. The minimum of the two ratios is then found and illustrated in the tree. Another feature available is ranking analysis. This procedure allows for the calculation of marginal cost, marginal effectiveness, and marginal cost-effectiveness at each decision node.

Once all base case analyses have been employed to derive the final outcome, sensitivity analysis can be used to test the robustness of the data. DATA allows for the calculation of one-way, two-way, and multi-way sensitivity analysis. Each result can be presented in graphic form with the indifference value indicated. The point of indifference, also called 
threshold value, indicates the value that renders no difference in cost-effectiveness between treatments. Given the availability of the software and its sufficient technical capabilities, it represented a viable choice for the completion of this study.

The primary objective at the outset of this project was to evaluate the potential of a new antidepressant herbal, St. John's Wort, in terms of its cost-effectiveness when used to treat mild to moderately depressed patients on an outpatient basis. According to the literature, the current standard of therapy for the treatment of these patients is fluoxetine. Its proven efficacy and popularity among practitioners is evidenced by its status as the number one prescribed antidepressant.

In order to maximize external validity, it is necessary to compare the therapy being questioned with its best alternative currently available (Luce and Simpson, 1995). Hence, fluoxetine was chosen as the alternative to which St. John's Wort was compared based on relative cost-effective ratios. This comparison was made under the assumption of specific treatment indications.

Treatment indication refers to the specific drug treatment regimen applied to a specific patient population (Eddy, 1992). Starting with initial treatment regimens, St. John's Wort is evaluated based on 300 milligrams $(\mathrm{mg})$ extract given three times daily while fluoxetine is evaluated on the initial dose of $20 \mathrm{mg}$ given once daily. The indication modeled in this study is the treatment of an adult patient, age 18 to 65 , with a first time diagnosis of mild to moderate depression with no suicidal ideation. The treatment is 
entirely on an outpatient basis only and is modeled for a period of one year, following the recommendations of the expert panel and the American Psychiatric Association (APA) that patients remain on therapy for a period of six to twelve months to decrease chance of relapse (APA, 1993). The perspective of the study is based on a third party payer viewpoint. Consequently, only direct costs of medical treatment encountered by patients are accounted for in the model and are described in detail in the cost analysis.

\section{MODEL DEVELOPMENT}

It was highly desirable to model the treatment of depression based on actual clinical practice patterns. Therefore, a panel of experts was identified to help establish the clinical management pathway for the outpatient treatment of depression that ultimately provided the structure of the decision tree (Figure 3.1).

Figure 3.1: Decision Tree for Treatment of Mild to Moderate Depression

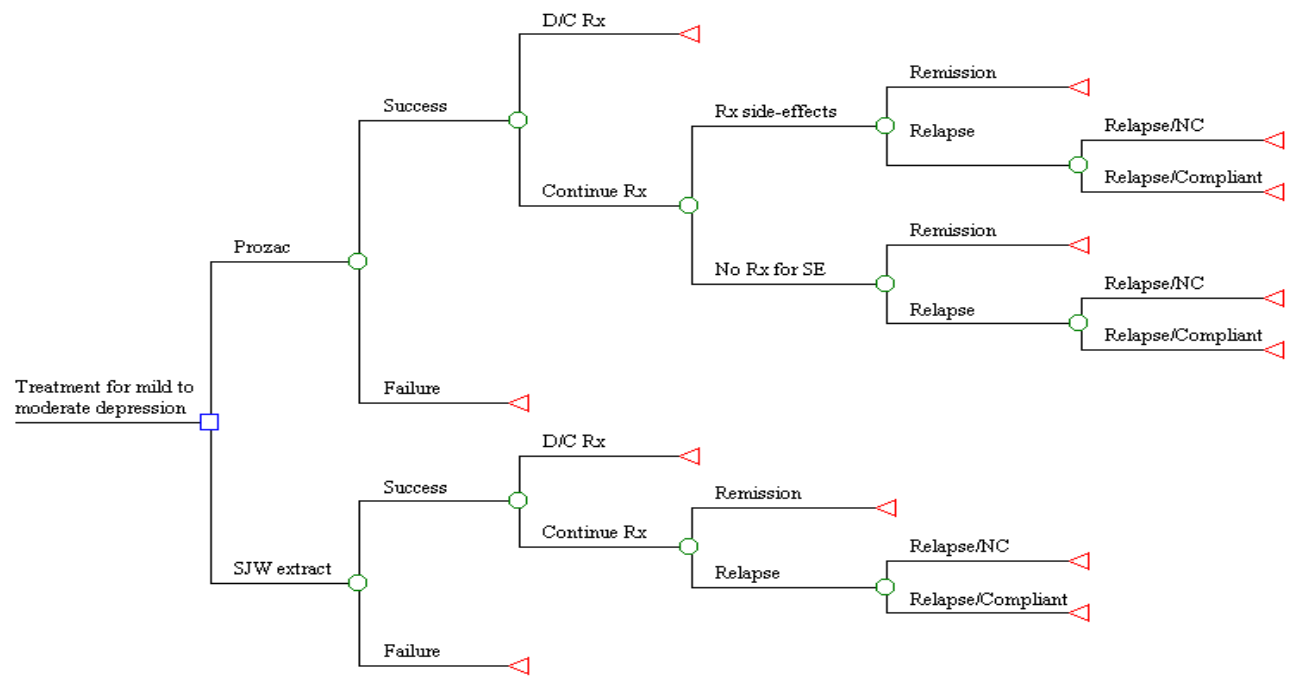

$\mathrm{D} / \mathrm{C}=$ discontinue; $\mathrm{NC}=$ non-compliant $; \mathrm{Rx}=$ treatment $\mathrm{SE}=$ side-effects; $\mathrm{SJW}=$ St. John's Wort 
The panel consisted of two psychiatrists, one pharmacist who specializes in psychiatry, and three primary care physicians all experienced in the diagnosis and management of major depressive disorders.

Panel members were asked several questions related to clinical practice and were instructed to base their answers on the following clinical scenario. A patient (nongeriatric, non-pediatric) presents to the outpatient setting with symptoms consistent with a diagnosis of major depressive disorder (MDD). This is the first episode experienced, and it is assessed to be of mild to moderate severity of illness with no suicidal ideation. Also, no comorbidities that would interfere with antidepressant drug therapy are present.

A modified delphi technique was used to gather information about the clinical management of patients (Fink, 1984). Results of the interviews were aggregated in tabular form. If total agreement was not reached, the majority response was used. The questionnaire and consensus responses are presented in Appendix A.

The choice of the alternative, fluoxetine, was agreed upon by the panel members as an appropriate comparator for the study. All agreed that SSRIs were the most commonly prescribed therapy and fluoxetine was a good representative for the class. Several of the panel members explained their rationale for choosing one drug over another in this class was usually based on the small differences in the side-effect profiles. The initial dose chosen was $20 \mathrm{mg}$ daily. This is supported by the package insert and the most common dose used in clinical trials. However, the panel estimated that approximately $25 \%$ of 
patients require a higher dose of $40 \mathrm{mg}$ daily. This was taken into consideration when determining cost of drug therapy and is presented in the cost analysis section.

The panel was then asked several questions regarding the management of patients diagnosed with depression. It was established that all patients would have a baseline thyroid stimulating hormone or TSH level drawn during the initial visit. The panel referred to the Diagnostic and Statistical Manual (DSM) as the primary criteria on which the diagnosis is made. The psychiatrists were more likely to rely on depression scales like the Bech-Rafaelsen Melancholia Scale and the Hamilton Depression Rating Scale (HAMD) to assess response, but in general all members agreed that the clinical interview evaluating the patient's self-assessment of improvement in DSM criteria was most often used. Definition of success as depicted in the model, however, is based on that defined in clinical trials as at least a 50\% reduction in baseline HAMD score.

Assessment of response and side-effects was determine to occur between two and four weeks. All members agreed that if an adequate response was not achieved, the patient would be switched to another SSRI. The model assumes treatment failures are switched to paroxetine (Paxil®). The panel agreed that this is a reasonable assumption given that clinical experience has shown that failure to respond to one SSRI does not preclude response to a different SSRI. Additionally, the APA guidelines support changing to a medication from the same class after initial failure to respond (APA, 1999). 
For both drugs, the panel estimated that the average number of physician visits a patient would have was six per year. The panel estimated that if a treatment failure or relapse occurred, the number of physician visits would increase to seven per year. For successfully treated patients, visits would occur at weeks two and four then at months four, six, nine, and twelve. For patients experiencing treatment failures, visits would occur at weeks two, four, and eight then at months four, six, nine, and twelve. All patients were assumed to have five psychotherapy visits, which involved individual counseling by an experienced psychologist. For treatment failures or relapses, psychotherapy visits increased to six per year. Success was defined as a $50 \%$ decrease in baseline score for the HAMD, which is called response rate in clinical trials. Failure, therefore is simply the complement of success (1- response rate).

Two possible outcomes could occur for patients achieving a successful response to therapy. Due to intolerable side-effects, patients would discontinue the drug for which drug treatment would then be changed to paroxetine. Paroxetine is consistently used to represent the second choice of drug therapy due to initial treatment failure, relapse, and discontinuations for both drugs. The panel agreed that an additional visit would be required for patients that failed to respond to initial therapy.

If the side-effects were tolerable, patients would continue treatment for six months. Many patients require additional therapy to combat side-effects. The most common sideeffect of fluoxetine that requires medical treatment, according to the panel, is anxiety that causes insomnia, which usually occurs within the first two weeks of therapy. Trazodone 
$50 \mathrm{mg}$ at bedtime is frequently prescribed for this side-effect. An assumption for those continuing treatment is that a percentage will require trazodone (fluoxetine arm only). Panel members cited trazodone as the most commonly prescribed treatment for sideeffects due to fluoxetine. Headache and gastro-intestinal upset were other common sideeffects reported. However, treatment for these adverse effects was assumed to be overthe-counter medications and therefore, was not included in the model.

At six months, a reassessment occurs and possible outcomes are remission, relapse due to noncompliance, and relapse due to ineffectiveness of treatment. The panel agreed that patients in remission would continue drug therapy at the same dose for an additional six months for a total of one year of drug therapy. One year was described by the expert panel as a standard length of therapy. The APA along with Agency for Health Care Policy and Research (AHCPR) guidelines suggest treatment of major depressive disorders be continued for a period of at least 16 to 20 weeks after the initial response has been achieved to minimize the chance of relapse. Hence, the model was based on one year of treatment in order to capture all relevant costs associated with treatment of this disorder, including relapse.

\section{EFFECTIVENESS DATA}

A search for published trials on St. John's Wort and fluoxetine was performed using MEDLINE, PSYCHLIT, EMBASE, and the Cochrane Database with the terms St. John's Wort, Hypericum, hypericin, fluoxetine, $\operatorname{Prozac} \AA$, depression, and depressive disorder 
along with the appropriate Boolean operators. Inclusion criteria used for the selection of effectiveness data were studies published in English that randomized patients between groups, in an outpatient setting, utilized a control group comparing either placebo or standard antidepressant therapy, assessed patients at least 18 years of age diagnosed with depression, defined response to treatment as $50 \%$ or more reduction in HAMD baseline score, and evaluated response over a period of at least four weeks.

Additionally, the minimum dose of fluoxetine accepted was $20 \mathrm{mg}$ daily. While no dose requirement was established for St. John's Wort, studies were limited to those testing single-entity products only. Exclusion criteria were studies testing severe depression, those involving an inpatient component, St. John's Wort products containing other active ingredients (combination products), and patients with co-morbid psychiatric conditions.

\section{EFFECTIVENESS OF ST. JOHN’ WORT}

The results of the search on St. John's Wort yielded 174 relevant records, of which 36 were clinical trials. A review of these records revealed that the majority of studies tested subjects with mild to moderately severe depression assessed by the HAMD scale. Most were clinical trials carried out on a small sample of patients classified with some type of depressive disorder according to ICD-9 (diagnostic) codes or DSM-III R criteria. A total of 32 trials met the inclusion criteria. Three studies comparing St. John's Wort to fluoxetine were identified. 
In the first study a total of 240 outpatients with initial HAMD scores between 16 and 24 were randomized to either $20 \mathrm{mg}$ fluoxetine daily or $250 \mathrm{mg}$ Ze117 twice daily (Schrader, 2000). The primary outcome measured was the change in mean HAMD score, measured at week zero and week six. Equivalence was defined prospectively as less than or equal to three points difference in mean score at endpoint for the two treatments. Intent-to-treat analyses were conducted on all variables.

The two treatments were determined to be equivalent based on the change in mean HAMD score of 7.25 (95\% Confidence Interval (CI): 8.1 to 6.4 ) for the fluoxetine group and $8.11(95 \%$ CI: 9.0 to 7.3$)$ for the extract group. Secondary variables measured were responder rate, compliance, mean clinical global impression (CGI), and safety. There were no differences noted in compliance rates, change in CGI for items 2 or 3 , or in patients' self-assessments. Significant differences were measured in responder rates ( $40 \%$ for fluoxetine and $60 \%$ for hypericum extract, $\mathrm{p}=0.05$ ) and mean change in item one of the CGI (1.3 for fluoxetine and 1.6 for the extract, $\mathrm{p}=0.03$ ). The most common adverse effect in the fluoxetine group was agitation occurring in $7.9 \%$ of patients, while gastrointestinal disturbances were most common in the extract group occurring in $4.8 \%$ of patients. Only one patient withdrew due to side-effects in the fluoxetine group.

The second and third studies were determined to be of little value due to the small sample size (Harrer, 1999 and Behnke, 2002). The lack of power precludes the ability to detect relatively small but clinically significant differences. Additionally, the more recent trial used a fluoxetine dose of $20 \mathrm{mg}$ twice daily, which is an unconventional regimen and the 
authors failed to report response rates. Therefore, the study failed to meet all the inclusion criteria.

Given that only one study comparing St. John's Wort to fluoxetine was identified and not all variables needed for the model were measured, it was decided to explore studies comparing different alternatives than those studied in this model (mainly St. John's Wort versus tricyclic antidepressants (TCA). It was particularly desirable to identify a metaanalysis that met the criteria previously established. In general, meta-analyses are preferred to a single clinical trial for use in decision analysis. A more heterogenous population and different clinical settings are represented, which aids in the generalizability of the results. Additionally, studies comparing St. John's Wort to standard antidepressants were given higher priority over placebo controlled trials due to the fact that St. John's Wort is modeled in this study as a potential substitute for current therapy and not as an adjunctive therapy. Three meta-anlyses were identified that met the inclusion criteria.

A meta-analysis by Whiskey et al. (2001) identified nine studies comparing St. John's Wort to standard antidepressants. Of those, four met the stricter methodological criteria of intent-to-treat analysis, sufficient power to detect statistical differences, and use of an effective dose of comparative antidepressant. The response rate ranged from $43 \%$ to $76 \%$ for St. John's Wort. The pooled number of drop-outs due to adverse drug reactions from all trials was $2 \%$ for St. John's Wort $(\mathrm{n}=689)$ and $10 \%$ for fluoxetine $(\mathrm{n}=79)$. 
Kim et al. (1999) performed a similar meta-analysis on four trials comparing St. John's Wort to TCAs. A pooled response rate of 64\% was derived for St. John's Wort and $66.4 \%$ for TCAs. One trial that did not include an intent-to-treat analysis was included which was excluded from the previous meta-analysis. Drop-out (discontinuation) rates were also reported as $12.6 \%$ for St. John's Wort due to side-effects or insufficient efficacy. Headache, gastrointestinal upset, and dizziness were the most commonly reported side-effects.

A meta-analysis by Linde et al. (1996) provided a comprehensive overview of randomized clinical trials that assessed the antidepressant effects of St. John's Wort against either placebo or standard TCAs. The results of the meta-anlysis provided four separate response rates. Studies were stratified based on control group (placebo or standard TCA) and hypericum (single source or combination) product. Within each of these categories, responder rates, dropout rates, and side-effects are reported.

The responder rate calculated from the three studies that compared single preparations of St. John's Wort to standard antidepressant therapies (amitriptyline, imipramine, and maprotiline) was $64 \%$ for St. John's Wort and 59\% for TCAs. The analysis by Kim in 1999 differed from this by only one study, which was published after Linde's analysis was conducted, yet yielded the same result in response rate. The drop-out rate for Hypericum groups was $4 \%$ and the percentage of patients reporting side-effects was $20 \%$. 
Since two of the meta-analyses yielded the same response rate and the third failed to report a pooled statistic, the response rate of $64 \%$ was chosen to represent the probability of successful treatment with St. John's Wort. This figure is relatively consistent with that reported by Schrader et al comparing St. John's Wort to fluoxetine. In that study, the response rate was $60 \%$. Therefore, it seems reasonable to use $64 \%$ given it is based on a larger data set. The response rate range ( $43 \%$ to $76 \%$ ) reported by Whiskey et al. was chosen to represent the range over which the sensitivity analysis for this variable was conducted.

Given the limited amount of data on St. John's Wort and the relative lack of clinical experience with the drug, conservative estimates (in terms of favorable outcomes) were chosen for most variables. The drop-out rate was chosen as $12.6 \%$ since this was the highest estimate reported. As reported in clinical trials, the most common side-effect is gastrointestinal upset relating to nausea, vomiting, abdominal discomfort and constipation. None of which would require prescription treatment. Therefore, the occurrence of side-effects for St. John's Wort was not included in the model as it is assumed not to have any associated direct medical costs from the payer's perspective. If however, these side-effects caused discontinuation of treatment, then the treatment strategy would be to change therapy. This is represented in the decision tree by probability of discontinuation of treatment.

There is a lack of long term data published on St. John's Wort. The literature search was unsuccessful in finding estimates on remission and/or relapse rates. Therefore, these data 
were based on the estimates obtained for fluoxetine. In each case, a more conservative estimate for St. John's Wort was assumed. Additionally, only limited information was found on compliance.

In general, patients are less compliant with thrice-daily regimens than once-daily regimens. This was evidence in a trial testing the affect number of doses has on compliance (Eisen, 1990). This study used a computerized device to record the number of times the medication container was opened. Three-time daily regimens were associated with more than two times the non-compliance rate than daily regimens. Hence, the value for relapse due to noncompliance for fluoxetine was doubled for St. John's Wort. A summary of the probability estimates is presented in Table 3.1.

Table 3.1: Base-case and range probability estimates for St. John's Wort

\begin{tabular}{|l|l|l|}
\hline PROBABILITY ESTIMATE & $\begin{array}{l}\text { ST. JOHN'S WORT } \\
(\text { RANGE } \%)\end{array}$ & DATA SOURCE \\
\hline SUCCESS & $64 \%(43-76)$ & META-ANALYSIS \\
\hline DISCONTINUATION OF RX & $13 \%(2-52)$ & META-ANALYSIS \\
\hline REMISSION & $40 \%(20-65)$ & N/A \\
\hline $\begin{array}{l}\text { RELAPSE DUE TO } \\
\text { NON-COMPLIANCE }\end{array}$ & $40 \%(10-85)$ & EISEN et al. 1990 \\
\hline
\end{tabular}

1 Kim et al. 1999; 2 Linde et al. 1996; $N / A=$ none available; $R X=$ treatment

\section{EFFECTIVENESS OF FLUOXETINE}

As a result of the literature search, it was apparent that effectiveness data on fluoxetine was more readily available than that on St. John's Wort. Fluoxetine has been on the market for more than a decade and is heavily relied on for its antidepressant effects. However, products containing St. John's Wort are fairly new to this country; its use as an 
antidepressant, until recently, have been virtually unknown. Consequently, it was highly desirable to find effectiveness data from studies that were similar in methodology to that of effectiveness data on St. John's Wort.

The abundance of randomized clinical trials testing the efficacy of fluoxetine for the treatment of depression involved both inpatients and outpatients with moderate to severe depression or other mental illnesses. Several meta-analyses were identified through the initial search. In developing clinical practice guidelines for the treatment of depression, the AHCPR conducted several meta-anlyses on newer antidepressant therapies including fluoxetine (AHCPR, 1993).

The response rate was reported for studies conducted on outpatients in primary care with MDD. Fluoxetine was reported to have a $47 \%$ response rate compared to other antidepressants. The range for all SSRIs tested was $43 \%$ to $59 \%$. Another meta-analysis conducted by Einarson et al. (1995) stratified studies based on clinical setting of inpatient or outpatient management of depression. They reported an overall success rate of $65 \%$ based on 12 fluoxetine trials.

A more recent review of antidepressant therapy was published as an evidence report summary (Williams, 2000). This review found no significant difference in efficacy between fluoxetine and other SSRIs. An average response rate of 54\% for newer antidepressants was reported, however, nothing specifically for fluoxetine. Drop-outs 
due to adverse effects were $11 \%$ for SSRIs. Relapse rates were also studied. Ten percent of patients on newer treatments relapsed within 24 weeks yielding a $90 \%$ remission rate.

In an effort to match the data obtained on St. John's Wort, it was highly desirable to identify trials testing fluoxetine in patients with mild to moderate depression. The search was successful in finding a meta-analysis that assessed response to fluoxetine based on severity of illness (Pande, 1993). This study re-analyzed data from 19 double-blinded randomized clinical trials of fluoxetine against placebo or TCA (amitriptyline, imipramine, doxepin, desipramine or nortriptyline).

Patients were stratified into three severity levels (mild, moderate, and severe depression) based on total HAMD scores at baseline. The results were then re-analyzed to yield response rates for each stratum. In all groups, fluoxetine was more effective than placebo with higher remission rates and as effective as TCAs with similar remission rates. The response rate for fluoxetine compared to TCAs was $77 \%$ for the mild depression group and $56 \%$ for the moderate depression group. The average of the two groups yields a response rate of $67 \%$. This study successfully illustrated the effectiveness of fluoxetine for all severity levels of MDD. No significant differences in efficacy were found between groups based on severity of illness. Given this important finding, use of the data obtained from the studies described above was deemed acceptable.

Overall, published clinical success rates for fluoxetine range from $40 \%$ to $77 \%$, which is used as the range over which the sensitivity analysis was performed. Given this disparity, 
the actual base case estimate used in the model was derived from the expert panel who reported an average of $63 \%$ of patients respond to therapy. This value is similar to that reported by an expert panel used in a similar cost-effectiveness study (Einarson, 1995).

A pragmatic trial was identified in the search that evaluated effectiveness and costs of fluoxetine in the outpatient setting compared to TCAs (Simon, 1996). The lack of a controlled setting provides results that are much more generalizable and therefore, relevant for inclusion in the decision analysis model. The rate of discontinuation due to adverse-effects was $9 \%$ for fluoxetine. Clinical remission was achieved by $49 \%$ of patients at six months for the fluoxetine group.

The best estimate found for remission rates was that published in the trial by Simon et al. (1996); $49 \%$ was used as the base estimate with a range of $35 \%$ to $90 \%$. The probability of a discontinuation due to adverse-effects was estimated to be $9 \%$ in the model, based on the same study. The expert panel however, estimated this to be approximately $20 \%$. Therefore, the estimate ranged from $2 \%$ (the average for placebo groups) to $20 \%$.

The probability of relapse is the compliment of the probability of remission. Therefore, the model estimated a $51 \%$ relapse rate for fluoxetine, ranging from $10 \%$ to $65 \%$. Compliance was built into the decision model as a component of relapse. No reliable estimate of compliance was identified in the records obtained. In the trial studying fluoxetine use in primary care, $61 \%$ of patients were compliant at three months (90 days) as measured by number of refills obtained (Simon, 1996). However, this measure of 
compliance is not always the most accurate as patients may hoard medications, use multiple pharmacies, and/or have access to sample medications.

Another study compared the effect of dosing regimens of anti-hypertensive medications on compliance rates (Eisen, 1990). This trial used computerized technology to count the number of times the medication bottle was opened and assumed each opening correlated to ingestion of the medication. Once-daily regimens were associated with an $84 \%$ rate of compliance while thrice-daily regimens fell to $59 \%$. It is reasonable to assume these rates will vary slightly depending on the medication being studied. Fluoxetine, though much better tolerated in clinical trials than tricyclics, still has a number of bothersome side-effects such as agitation, headache, nausea, sexual dysfunction, and weight gain. Hence, the relapse rate due to non-compliance was assumed to be $20 \%$ as compared to $16 \%$ found for once-daily regimens. This figure was ranged from $5 \%$ to $40 \%$.

The last variable, probability of agitation, was based on the rate published in the Physicians Desk Reference of $20 \%$. The expert panel estimated a similar rate of occurrence. However, since the model assumed that every occurrence would require treatment, the more conservative estimate of $10 \%$ was used for the base case analysis. This base estimate was varied from $2 \%$ to $20 \%$. A summary of effectiveness variables for fluoxetine is presented in Table 3.2. 
Table 3.2: Base-case and range probability estimates for fluoxetine

\begin{tabular}{|l|l|l|}
\hline PROBABILITY ESTIMATE & $\begin{array}{l}\text { FLUOXETINE } \\
(\text { RANGE } \%)\end{array}$ & DATA SOURCE \\
\hline SUCCESS & $63 \%(40-77)$ & EXPERT PANEL \\
\hline DISCONTINUATION OF RX & $9 \%(2-20)$ & SIMON et al., 1996 \\
\hline SIDE-EFFECT RX & $10 \%(2-20)$ & PDR; EXPERT PANEL \\
\hline REMISSION & $49 \%(35-90)$ & SIMON et al., 1996 \\
\hline $\begin{array}{l}\text { RELAPSE DUE TO } \\
\text { NON-COMPLIANCE }\end{array}$ & $20 \%(5-40)$ & EISEN et al., 1990 \\
\hline
\end{tabular}

$\mathrm{PDR}=$ Physicians Desk Reference, 2002; RX=treatment

\section{COST DATA}

Cost information for direct medical care was based on charges obtained from a primary care clinic in Guilford, Connecticut as well as an outpatient psychiatry clinic in New Haven, Connecticut. Direct medical care included an initial evaluation and follow-up visits to a primary care physician, an initial evaluation and follow-up visits to a clinical psycologist for individual psychotherapy sessions, and a baseline thyroid stimulating hormone (TSH) level.

Drug acquisition cost was based on 2002 Red Book Average Wholesale Prices (AWP) for generic trazodone $50 \mathrm{mg}$, and brand Paxil® $20 \mathrm{mg}$ and $30 \mathrm{mg}$. The cost of generic fluoxetine $20 \mathrm{mg}$ and $40 \mathrm{mg}$ was based on data from retail pharmacies nationwide provided by Verispan, SPA for the time period of September 2001 to August, 2002 in addition to the published AWP for generic fluoxetine. The panel estimated that approximately $25 \%$ of patients require a dosage increase. 
To account for this, the cost of therapy for one week of Paxil $®$ was based on $75 \%$ of the AWP for one week of $20 \mathrm{mg}$ and $25 \%$ of the AWP for one week of $30 \mathrm{mg}$.

Example calculation: The average AWP for a $20 \mathrm{mg}$ tablet of Paxil $\AA=\$ 2.76$

The average AWP for a $30 \mathrm{mg}$ tablet of Paxil ${ }^{\circledR}=\$ 2.79$

$$
(\$ 2.76 * 75 \%)+(\$ 2.79 * 25 \%)=\$ 2.77 \text { or } \$ 19.37 \text { per week of therapy }
$$

The lower end of the cost range for fluoxetine was determined by using the average AWP for generic fluoxetine and estimating that only $25 \%$ of this price would be paid by the insurer. Third-party payers rarely reimburse $100 \%$ of the AWP as this price is inflated compared to the acquisition cost to the pharmacy.

Example calculation: The median generic AWP for a $20 \mathrm{mg}$ tablet of fluoxetine $=\$ 2.67$

$$
\$ 2.67 * 25 \%=\$ 0.67 \text { day or } \$ 4.67 \text { per week of therapy }
$$

The highest end of the cost range for fluoxetine was determined by using the average AWP for brand fluoxetine and estimating that $25 \%$ of patients require $40 \mathrm{mg}$ daily while $75 \%$ require $20 \mathrm{mg}$ daily.

Example calculation: The median AWP for a $20 \mathrm{mg}$ tablet of Prozac $\AA=\$ 3.55$

The median AWP for a $40 \mathrm{mg}$ tablet of $\operatorname{Prozac} \AA=\$ 7.10$

$$
(\$ 3.55 * 75 \%)+(\$ 7.10 * 25 \%)=\$ 4.44 / \text { day or } \$ 31.08 \text { per week of therapy }
$$


Cost of a 30-day supply of St. John's Wort $300 \mathrm{mg}$ capsules taken three times per day was based on the average price of six different brands sold at various retailers in Hamden and Cheshire, Connecticut. Total cost of drug therapy for the year was estimated by multiplying the average weekly cost by the number of weeks of therapy. Tables 3.3, 3.4, and 3.5 provide a summary of the drug cost data.

Table 3.3: Direct medical costs associated with treatment of depression

\begin{tabular}{|l|c|l|}
\hline VARIABLE & COST \$ (RANGE) & DATA SOURCE \\
\hline TSH LEVEL & 25.00 & PCC \\
\hline MD VISIT (INITIAL) & 145.00 & PCC \\
\hline MD VISIT (FOLLOW-UP) & 85.00 & PCC \\
\hline PSYCOTHERAPY (INITIAL) & 115.00 & OPC \\
\hline PSYCOTHERAPY (FOLLOW-UP) & 75.00 & OPC \\
\hline FLUOXETINE & $15.58^{\wedge}(4.67-31.08)^{*}$ & $\begin{array}{l}\text { ARP; Red Book, } \\
\text { 2002 AWP }\end{array}$ \\
\hline ST. JOHN'S WORT & $3.68^{\wedge}(1.35-20.00)$ & ARP \\
\hline PAXIL & $19.37^{\wedge *}$ & $\begin{array}{l}\text { Red Book, } 2002 \\
\text { AWP }\end{array}$ \\
\hline & & $\begin{array}{l}\text { Red Book, } 2002 \\
\text { AWP }\end{array}$ \\
\hline
\end{tabular}

$\mathrm{ARP}=$ average retail price of six different brands; $\mathrm{AWP}=$ average wholesale price; $\mathrm{mg}=$ miligrams; OPC $=$ Outpatient Psychiatry Clinic; $P C C=$ Primary Care Clinic

$\wedge^{\wedge}$ one week of therapy, *based on AWP calculation shown in previous section

Table 3.4: Average retail price of 30-day supply of St. John's Wort $900 \mathrm{mg}$

\begin{tabular}{|c|c|}
\hline ST. JOHN'S WORT (\$) & SOURCE* \\
\hline 5.79 & PHARMAPURE \\
\hline 6.99 & VITAMIN WORLD \\
\hline 11.97 & GNC \\
\hline 13.99 & GNC, ZE 117 \\
\hline 16.18 & HOMEBEST \\
\hline 20.58 & KIRA, LI 160 \\
\hline
\end{tabular}

*Retailers located in Cheshire, Connecticut, November 2002 
Table 3.5: Average retail price of 30-day supply of fluoxetine $20 \mathrm{mg}$

\begin{tabular}{|c|c|}
\hline FLUOXETINE COST (\$) & SOURCE* $^{*}$ \\
\hline 49.68 & WAL-MART $^{\wedge}$ \\
\hline 53.40 & VERISPAN $^{\wedge}$ \\
\hline 54.99 & WALGREENS \\
\hline 74.99 & RITE-AID \\
\hline 78.99 & CVS PHARMACY \\
\hline 82.49 & BROOKS PHARMACY \\
\hline
\end{tabular}

*Retailers located in Cheshire, Connecticut, November 2002; ${ }^{\wedge}$ Verispan provides cost information from retail pharmacies nationwide, compiled from September, 2001 to August, 2002

The expert panel estimated utilization of medical care for each pathway represented in the decision model. For Path 1, a patient successfully responds to initial therapy with fluoxetine, then discontinues therapy due to adverse drug reactions. An initial physician evaluation is performed then a follow-up visit is required to change therapy. The patient is assumed to have five additional follow-up visits. Additionally, the patient has five psychotherapy sessions, one initial evaluation and four subsequent visits. Drug costs for this path involves four weeks of fluoxetine and 48 weeks of paroxetine (Paxil@).

Path 2 involves the same scenario for patients initially responding to fluoxetine, then continuing therapy but requiring treatment for side-effects, then subsequently going into remission for the remainder of the year. The number of physician visits as well as psychotherapy visits remains the same as Path 1 . The expert panel estimated an average of six physician visits per year would be encountered by patients. The occurrence of side-effects requiring treatment or the need to change therapy would require an additional visit. 
The drug costs are represented by 52 weeks of fluoxetine, assuming full compliance, and 50 weeks of trazodone. The expert panel estimated that the majority of side-effects occur within the first two weeks of therapy. Another assumption of the model is that patients are fully compliant with the medication used to treat the side-effect.

Path 3 is similar to Path 2 except that the patient experiences a relapse by month six due to medication non-compliance. Generally, non-compliance is defined as taking less than $70 \%$ of the prescribed medication. Therefore, Path 3 assumes the patient is only $69 \%$ compliant with fluoxetine and trazodone both. Because relapse is based on noncompliance in this path, the model assumes that fluoxetine will again be prescribed and taken for an additional six months at full compliance. This yields a total of 44 weeks of fluoxetine and 34.5 weeks of trazodone costs.

Based on the expert panel, the patient would have an additional visit to re-evaluate response and emphasize the importance of compliance. Hence, eight total physician visits and five psychotherapy visits are associated with this path. Conversely, Path 4 assumes the patient relapsed despite full compliance. Therefore, therapy is changed to paroxetine $\left(\right.$ Paxil $\left.{ }^{\circledR}\right)$ and full compliance is again assumed. A full 26 weeks of fluoxetine, 24 weeks of trazodone (for the first six months), and 26 weeks of paroxetine are required in Path 4. 
Paths 5, 6, and 7 are virtually the same as 2, 3, and 4 respectively. However, since these paths assume no treatment is required for side-effects, the cost of an additional physician visit is not present, nor is the cost of trazadone. All else remains the same.

The last path in the fluoxetine arm, 8, represents treatment failures. As discussed previously, patients failing to respond by week four to fluoxetine are switched to paroxetine $($ Paxil $®)$. It is assumed that these patients are more treatment resistant and will require a higher dose of paroxetine. Therefore, the cost of $30 \mathrm{mg}$ Paxil ${ }^{\circledR}$ was used in this path as opposed to the mix of $75 \%$ of the $20 \mathrm{mg}$ daily cost and $25 \%$ of the $30 \mathrm{mg}$ cost being used.

All assumptions hold true for the St. John's Wort arm of the model. The main difference is the omission of the side-effect treatment outcome. This was omitted due to the type of adverse effects reported. The most commonly reported effects such as headache and gastrointestinal upset do not require treatment with a prescription medication. Rather they are usually self-limiting and/or managed with over-the-counter medications.

The only cost difference in this arm is that of the extract itself, which would be substituted for the cost of fluoxetine in Paths $1,5,6,7$, and 8 . To remain consistent across treatment groups, a similar assumption was applied to the cost of the extract. Based on the expert panel's estimate of $25 \%$ of patients requiring a higher dose of fluoxetine, this condition was placed on St. John's Wort. Cost of therapy was estimated from $75 \%$ of the average cost of a 30 -day supply of $300 \mathrm{mg}$ capsules three times a day plus $25 \%$ of $600 \mathrm{mg}$ capsules three times a day. 


\section{ANALYTIC STRATEGY}

A total cost associated with each drug was evaluated without respect to effectiveness data. Expected cost-effectiveness was then calculated for each arm of the decision tree by folding back the tree. Once the base case analysis was performed, sensitivity analyses was performed on several variables. A one-way sensitivity analysis was performed on the response (success) rate of fluoxetine since there was not consensus in the literature on the value of this variable. The probability of successful treatment for the fluoxetine arm was varied from $40 \%$ to $77 \%$. These values were derived from the published metaanalyses identified in the literature search. Similarly, the response rate for St. John's Wort was also varied from $43 \%$ to $76 \%$.

Given that acquisition cost usually plays a large role in choice of therapy, the cost of fluoxetine as well as St. John's Wort was analyzed to determine at what cost, if any, a difference in cost-effectiveness may be seen. The other variables that were tested were those that were not well supported in the literature nor well defined by the expert panel. These include rates of discontinuation, relapse/remission, and non-compliance for both therapies. A threshold analysis was reported for variables that proved to be important in terms of its affect on the overall result. Two-way sensitivity analyses were also conducted to better analyze the effect of changing the value of multiple variables. 


\section{CHAPTER 4}

\section{RESULTS}

\section{INTRODUCTION}

A cost-effective analysis was performed based on the principles of decision analysis to determine optimal choice of therapy for treatment of mild to moderate depression. The perspective of the study is that of a third-party payer and takes into account direct medical expenses incurred by the payer in the outpatient setting. The costs and outcomes of fluoxetine and St. John's Wort are being compared by means of a decision tree model that typifies clinical management of the disease. This model was developed based on the results of the survey (Appendix A) of an expert panel of practitioners. The results of each objective are presented below.

\section{TOTAL COST OF THERAPY}

Objective A. Calculate the expected cost associated with the treatment of mild to moderate depression using fluoxetine at the initial dose of $20 \mathrm{mg}$ once a day.

The total cost for each pathway using fluoxetine in the decision tree is presented in Table 4.1. The least costly pathway in the fluoxetine arm ( $\$ 1,820 /$ patient $)$ is represented by a successful response that does not require treatment for side effects followed by remission requiring six months more of maintenance therapy. The possibility of a successful 
response requiring treatment for side effects followed by a relapse requiring a change of drug therapy is the most costly for fluoxetine ( $\$ 2,248 /$ patient).

Table 4.1 Total $\operatorname{cost}^{\mathrm{a}}$ of care for fluoxetine

\begin{tabular}{|c|c|c|c|c|c|c|c|c|}
\hline Fluoxetine & $\begin{array}{l}\text { PATH } \\
1\end{array}$ & $\begin{array}{l}\text { PATH } \\
2\end{array}$ & $\begin{array}{l}\text { PATH } \\
3\end{array}$ & $\begin{array}{l}\text { PATH } \\
4\end{array}$ & $\begin{array}{l}\text { PATH } \\
5\end{array}$ & $\begin{array}{l}\text { PATH } \\
6\end{array}$ & $\begin{array}{l}\text { PATH } \\
7\end{array}$ & $\begin{array}{l}\text { PATH } \\
8\end{array}$ \\
\hline MD eval & $\begin{array}{l}\$ 145 \\
(1)\end{array}$ & $\begin{array}{l}\$ 145 \\
(1)\end{array}$ & $\begin{array}{l}\$ 145 \\
(1)\end{array}$ & $\begin{array}{l}\$ 145 \\
(1)\end{array}$ & $\begin{array}{l}\$ 145 \\
(1)\end{array}$ & $\begin{array}{l}\$ 145 \\
(1)\end{array}$ & $\begin{array}{l}\$ 145 \\
\text { (1) }\end{array}$ & $\begin{array}{l}\$ 145 \\
\text { (1) }\end{array}$ \\
\hline MD f/u & $\begin{array}{l}\$ 85 \\
(6)\end{array}$ & $\begin{array}{l}\$ 85 \\
(6)\end{array}$ & $\begin{array}{l}\$ 85 \\
(7)\end{array}$ & $\begin{array}{l}\$ 85 \\
(7)\end{array}$ & $\begin{array}{l}\$ 85 \\
(5)\end{array}$ & $\$ 85(6)$ & $\$ 85(6)$ & $\begin{array}{l}\$ 85 \\
(6)\end{array}$ \\
\hline Psych eval & $\begin{array}{l}\$ 115 \\
(1)\end{array}$ & $\begin{array}{l}\$ 115 \\
(1)\end{array}$ & $\begin{array}{l}\$ 115 \\
(1)\end{array}$ & $\begin{array}{l}\$ 115 \\
(1)\end{array}$ & $\begin{array}{l}\$ 115 \\
(1)\end{array}$ & $\begin{array}{l}\$ 115 \\
\text { (1) }\end{array}$ & $\begin{array}{l}\$ 115 \\
\text { (1) }\end{array}$ & $\begin{array}{l}\$ 115 \\
(1)\end{array}$ \\
\hline Psych f/u & $\begin{array}{l}\$ 75 \\
\text { (4) }\end{array}$ & $\begin{array}{l}\$ 75 \\
(4)\end{array}$ & $\begin{array}{l}\$ 75 \\
(5)\end{array}$ & $\begin{array}{l}\$ 75 \\
(5)\end{array}$ & $\begin{array}{l}\$ 75 \\
(4)\end{array}$ & $\$ 75(5)$ & $\$ 75(5)$ & $\begin{array}{l}\$ 75 \\
(5)\end{array}$ \\
\hline TSH & $\begin{array}{l}\$ 25 \\
(1)\end{array}$ & $\begin{array}{l}\$ 25 \\
(1)\end{array}$ & $\begin{array}{l}\$ 25 \\
(1)\end{array}$ & $\begin{array}{l}\$ 25 \\
(1)\end{array}$ & $\begin{array}{l}\$ 25 \\
(1)\end{array}$ & $\$ 25(1)$ & $\$ 25(1)$ & $\begin{array}{l}\$ 25 \\
(1)\end{array}$ \\
\hline Fluoxetine $^{b}$ & $\begin{array}{l}\$ 15.5 \\
8(4)\end{array}$ & $\begin{array}{l}\$ 15.58 \\
(52)\end{array}$ & $\begin{array}{l}\$ 15.58 \\
(44)\end{array}$ & $\begin{array}{l}\$ 15.58 \\
(26)\end{array}$ & $\begin{array}{l}\$ 15.58 \\
(52)\end{array}$ & $\begin{array}{l}\$ 15.58 \\
\text { (44) }\end{array}$ & $\begin{array}{l}\$ 15.58 \\
(26)\end{array}$ & $\begin{array}{l}\$ 15.58 \\
\text { (4) }\end{array}$ \\
\hline Trazodone $^{b}$ & $\begin{array}{l}\$ 3.50 \\
(2)\end{array}$ & $\begin{array}{l}\$ 3.50 \\
(50)\end{array}$ & $\begin{array}{l}\$ 3.50 \\
(34.5)\end{array}$ & $\begin{array}{l}\$ 3.50 \\
(24)\end{array}$ & $\begin{array}{l}\$ 3.50 \\
(0)\end{array}$ & $\begin{array}{l}\$ 3.50 \\
(0)\end{array}$ & $\begin{array}{l}\$ 3.50 \\
(0)\end{array}$ & $\begin{array}{l}\$ 3.50 \\
(2)\end{array}$ \\
\hline Paroxetine $^{b}$ & $\begin{array}{l}\$ 19.3 \\
7(48)\end{array}$ & $\begin{array}{l}\$ 19.37 \\
(0)\end{array}$ & $\begin{array}{l}\$ 19.37 \\
(0)\end{array}$ & $\begin{array}{l}\$ 19.37 \\
(26)\end{array}$ & $\begin{array}{l}\$ 19.37 \\
(0)\end{array}$ & $\begin{array}{l}\$ 19.37 \\
(0)\end{array}$ & $\begin{array}{l}\$ 19.37 \\
(26)\end{array}$ & $\begin{array}{l}\$ 19.53 \\
(48)\end{array}$ \\
\hline TOTAL & $\$ 2094$ & $\$ 2080$ & $\$ 2061$ & $\$ 2248$ & $\$ 1820$ & $\$ 1856$ & $\$ 2079$ & $\$ 2177$ \\
\hline
\end{tabular}

${ }^{a}$ Dollar value is the cost per unit; Number of units incurred is in parentheses.

${ }^{b}$ Drug costs represent cost per week of therapy.

MD eval=initial physician evaluation; MD f/u=physician follow-up visits; Psych eval=initial psychotherapy evaluation; Psych f/u=psychotherapy follow-up visits; TSH=thyroid stimulating hormone level

Objective B. Calculate the expected cost associated with the treatment of mild to moderate depression using St. John's Wort at the initial dose of 300 mg three times a day.

The total cost for each pathway using St. John's Wort in the decision tree is presented in Table 4.2. Similar to fluoxetine, the least costly pathway in the St. John's Wort arm ( $\$ 1,201 /$ patient) is represented by a successful response followed by remission requiring 6 months of maintenance therapy. However, the most costly pathway differs from that of 
fluoxetine. For St. John's Wort, the pathway representing treatment failure requiring a change of drug therapy is the most costly possibility ( $\$ 2,122 /$ patient).

Table 4.2 Total cost ${ }^{\mathrm{a}}$ of care for St. John's Wort

\begin{tabular}{|c|c|c|c|c|c|}
\hline St. John's Wort & PATH 9 & PATH 10 & PATH 11 & PATH 12 & PATH 13 \\
\hline MD eval & $\$ 145(1)$ & $\$ 145(1)$ & $\$ 145(1)$ & $\$ 145(1)$ & $\$ 145(1)$ \\
\hline MD f/u & $\$ 85(6)$ & $\$ 85(5)$ & $\$ 85(6)$ & $\$ 85(6)$ & $\$ 85(6)$ \\
\hline Psych eval & $\$ 115(1)$ & $\$ 115(1)$ & $\$ 115(1)$ & $\$ 115(1)$ & $\$ 115(1)$ \\
\hline Psych f/u & $\$ 75(4)$ & $\$ 75(4)$ & $\$ 75(5)$ & $\$ 75(5)$ & $\$ 75(5)$ \\
\hline TSH & $\$ 25(1)$ & $\$ 25(1)$ & $\$ 25(1)$ & $\$ 25(1)$ & $\$ 25(1)$ \\
\hline Extract $^{b}$ (St. John's Wort) & $\$ 3.68(4)$ & $\begin{array}{l}\$ 3.68 \\
(52)\end{array}$ & $\begin{array}{l}\$ 3.68 \\
(18)\end{array}$ & $\begin{array}{l}\$ 3.68 \\
(26)\end{array}$ & $\$ 3.68(4)$ \\
\hline Paroxetine $^{b}$ & $\begin{array}{l}\$ 19.37 \\
(48)\end{array}$ & $\begin{array}{l}\$ 19.37 \\
(0)\end{array}$ & $\begin{array}{l}\$ 19.37 \\
(26)\end{array}$ & $\begin{array}{l}\$ 19.37 \\
(26)\end{array}$ & $\begin{array}{l}\$ 19.53 \\
(48)\end{array}$ \\
\hline TOTAL & $\$ 2039$ & $\$ 1201$ & $\$ 1740$ & $\$ 1769$ & $\$ 2122$ \\
\hline
\end{tabular}

${ }^{a}$ Dollar value is the cost per unit; Number of units incurred is in parentheses.

${ }^{b}$ Drug costs represent cost per week of therapy.

MD eval=initial physician evaluation; MD f/u=physician follow-up visits; Psych eval=initial psychotherapy evaluation; Psych f/u=psychotherapy follow-up visits; TSH=thyroid stimulating hormone level

\section{COST-EFFECTIVENESS}

Objective C. Assess the overall cost per successfully treated patient (costeffectiveness) of St. John's Wort.

Objective D. Assess the overall cost per successfully treated patient (costeffectiveness) of fluoxetine.

Objective E. Assess the incremental cost-effectiveness of St. John's Wort compared to fluoxetine.

When the tree is rolled back, the base case analysis reveals St. John's Wort to be the most cost-effective treatment for mild to moderate depression over a period of one year based on a third party perspective (Appendix B). The one-year cost per successfully treated 
patient with St. John's Wort is $\$ 1,788$ compared to $\$ 2,040$ with fluoxetine . This results in an incremental cost-effectiveness ratio of $\$ 252$ per patient per year.

\title{
SENSITIVITY ANALYSES
}

\author{
Objective F. Assess the robustness of the findings by performing sensitivity \\ analyses.
}

The robustness of this result was tested by performing several univariate and multivariate sensitivity analyses. Variables that were not well represented in the literature, had a wide range of published values and those for which consensus was not reached by the expert panel were chosen to analyze. In total, a two-way sensitivity analysis and 12 oneway sensitivity analyses were performed on the following variables for each therapy: probability of success (measured by response rate), cost of therapy, probability of remission, probability of relapse from noncompliance, probability of agitation as a sideeffect, and probability of discontinuation.

The one-way sensitivity analysis on probability of success with fluoxetine was performed by varying the success rate from $40 \%$ to $77 \%$ and maintaining St. John's Wort at $64 \%$ success rate. This range was chosen based on the highest and lowest reported values in the literature. The results of the analysis illustrate that St. John's Wort remains more cost-effective than fluoxetine at any value in the given range (Graph 4.1). In fact, the slope of the line depicting the expected value for fluoxetine suggests that even success 
rates greater than $77 \%$ would not change the expected value enough to make fluoxetine a better (more cost-effective) choice than St. John's Wort.

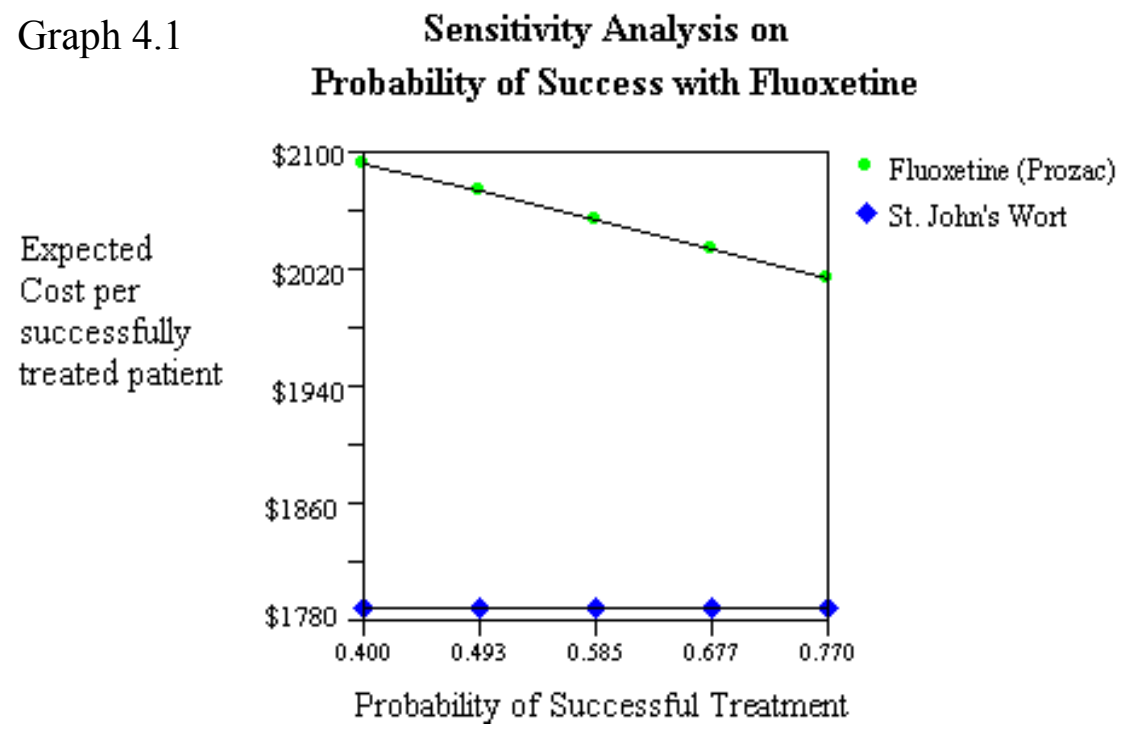

The results were then tested in a one-way sensitivity analysis based on the value of St.

John's Wort success rate. This value was ranged from $43 \%$ to $76 \%$ based on published rates in the literature while keeping fluoxetine's success rate constant at $63 \%$, the base case estimate. The results of the base case analysis were not affected by changing the rate of success for St. John's Wort (Graph 4.2). At all values, St. John's Wort was shown more cost-effective than fluoxetine. Hence, the results are very insensitive to the actual response rate of each therapy. 


\section{Graph 4.2 Sensitivity Analysis on \\ Probability of Success on St. John's Wort}

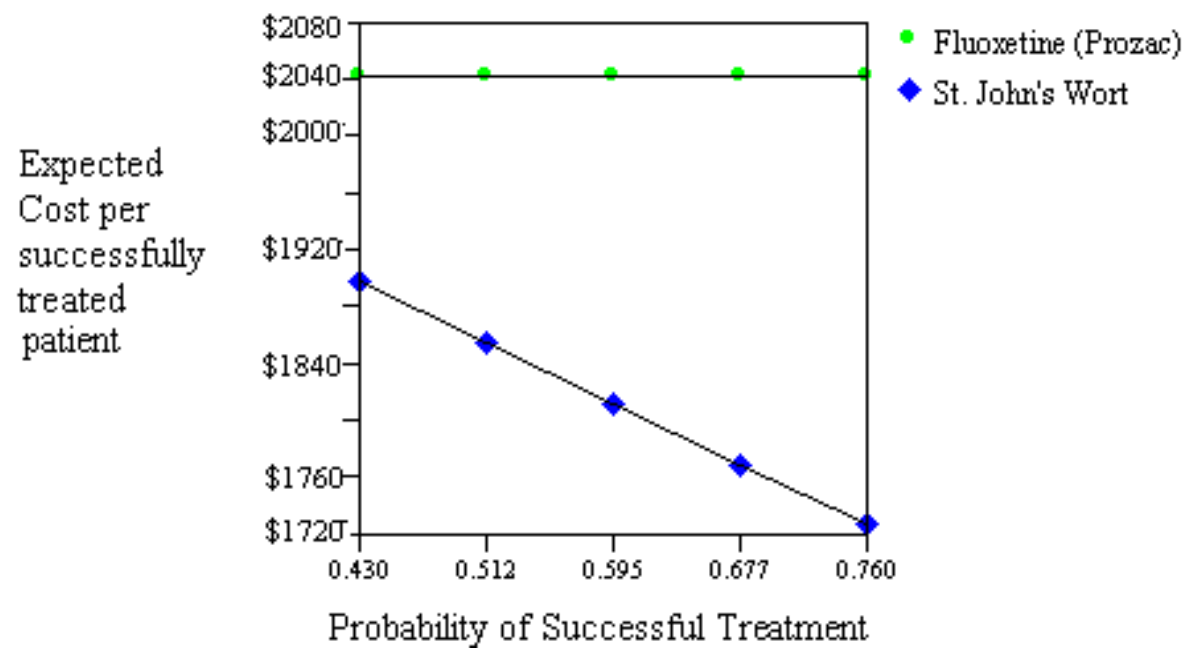

The analysis was then repeated to determine if a lower success rate would affect the results. The analysis was performed for the range of $25 \%$ to $76 \%$ while maintaining fluoxetine at $63 \%$ (graph 4.3). Once again, the expected cost of St. John's Wort remained lower than fluoxetine making it the more cost-effective alternative. 


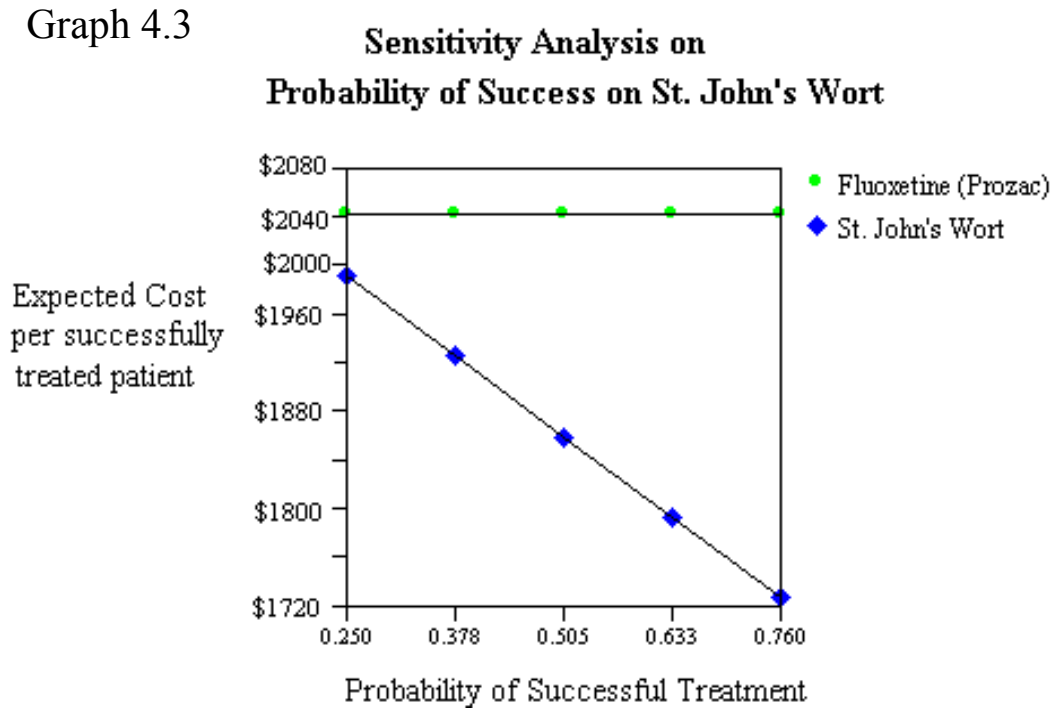

Cost plays a large role in the initial choice of therapy. Fluoxetine's patent recently expired, and several generic products are currently marketed at substantially lower prices than Eli Lilly's brand of Prozac ${ }^{\circledR}$. Given that this variable has such an integral role in choice of therapy in clinical practice, it was of interest to test its effect on total cost of care as modeled in the decision tree. A sensitivity analysis was performed on both the cost of fluoxetine and the cost of St. John's Wort.

The first analysis tested the effect of varying the cost of a one-week supply of fluoxetine from $\$ 4.67$ ( $\$ 20.01$ per 30-day supply) to $\$ 31.08$ ( $\$ 133.11$ per 30-day supply) while maintaining the weekly cost of $\$ 3.68$ for St. John's Wort. The range for fluoxetine's cost was chosen to represent the likely cost for the generic product once competition drives down the price and the highest probable cost for the brand product. Results of this analysis (Graph 4.4) demonstrate that drug acquisition cost does affect the overall costeffectiveness of therapy. In this analysis, the most cost-effective therapy changes from St. John's Wort to fluoxetine once the cost per week of fluoxetine is below $\$ 5.40$. At the 
threshold value of $\$ 5.40$ per week of fluoxetine, cost-effectiveness is equivalent, rendering the decision-maker indifferent to the alternatives.

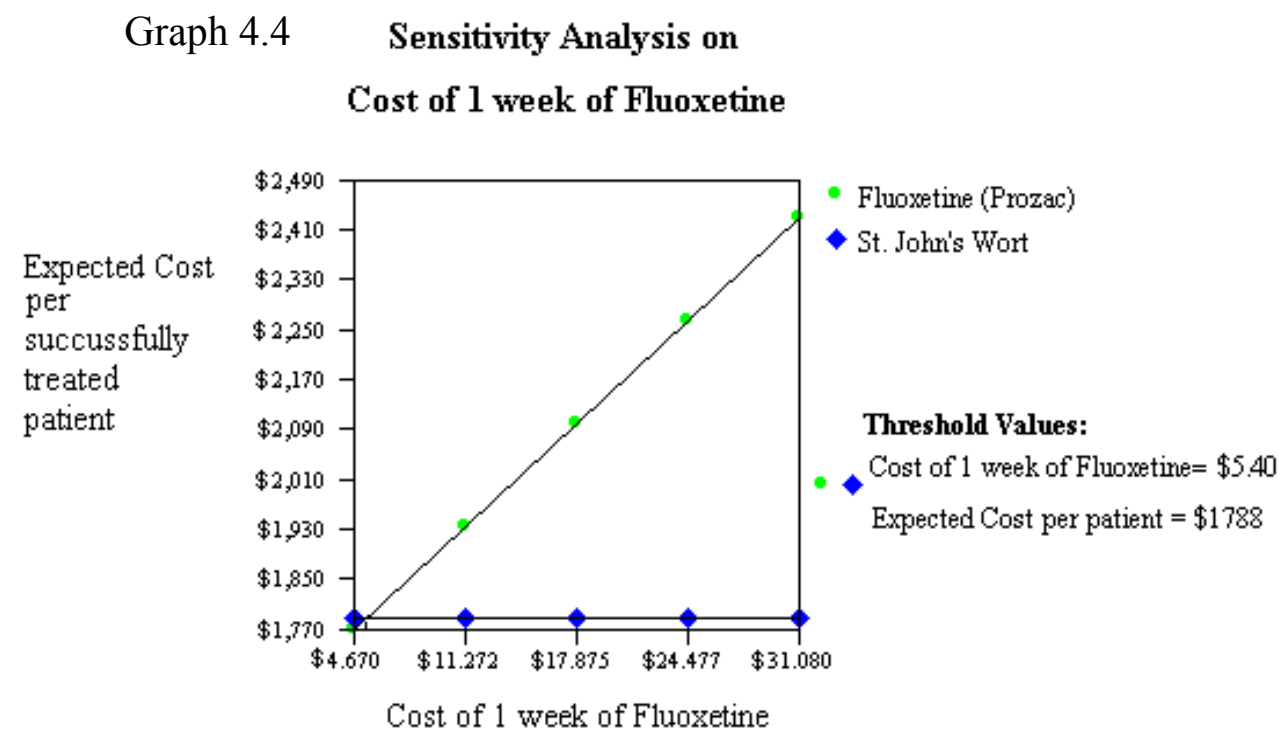

A one-way sensitivity analysis was repeated on the cost of a one-week supply of St.

John's Wort (Graph 4.5). The analysis held fluoxetine's weekly price constant at $\$ 15.58$ while St. John's Wort was varied over the weekly cost range of \$1.35 (\$5.79 per 30-day supply) to $\$ 20.00$ (\$85.71 per 30-day supply). These figures represents those obtained from 6 different brands sold by various retailers including GNC, Vitamin World, and Brooks Pharmacy located in Connecticut. The resultant graph depicts a point of indifference where both options are equally cost-effective. At any cost above $\$ 15.80$ per week for St. John's Wort, fluoxetine is a more cost-effective alternative. Both graphs 4 and 5 illustrate that drug acquisition cost plays an important role in the overall cost of therapy and the results of the base case analysis are relatively sensitive to this variable. 


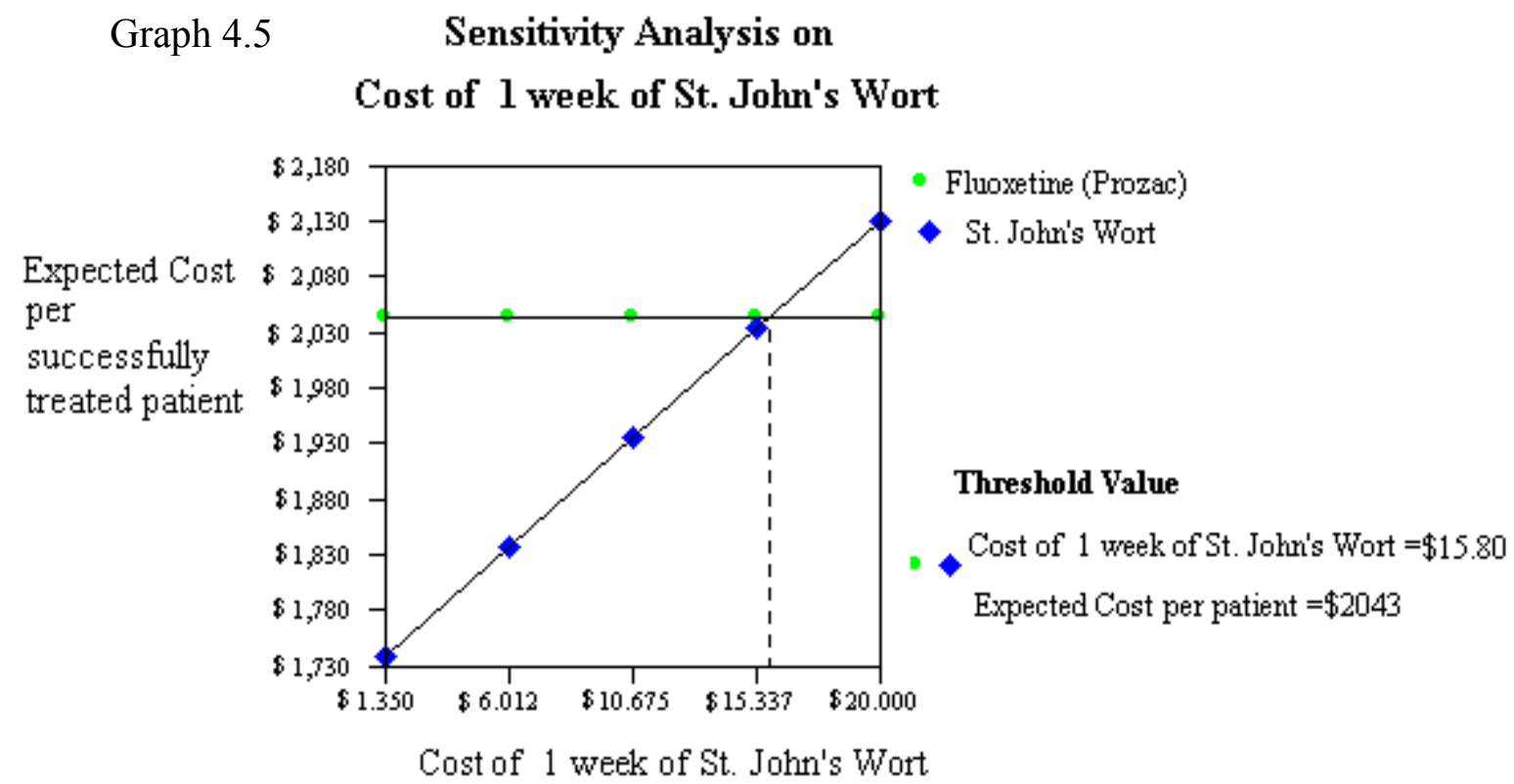

Given that the cost of either therapy affects the decision made in this model, a two-way sensitivity analysis was performed to better evaluate the effect of drug cost on choice of therapy. Graph 4.6 represents the effect of cost of therapy on the optimal decision. The graph is divided into two sections. The light gray section represents all costs for each therapy that result in St. John's Wort being more cost-effective while the dark area in the bottom right side of the graph depicts all costs that yield fluoxetine as the most costeffective therapy. At the lowest cost over which both St. John's Wort and fluoxetine were varied, St. John's Wort remains the most cost-effective choice. However, at the highest cost for St. John's Wort, it is only the best choice if fluoxetine's cost is more than 
approximately $\$ 18.00$ per week. Once fluoxetine drops below 18.00 per week, St. John's Wort is no longer the most cost-effective option at that price.

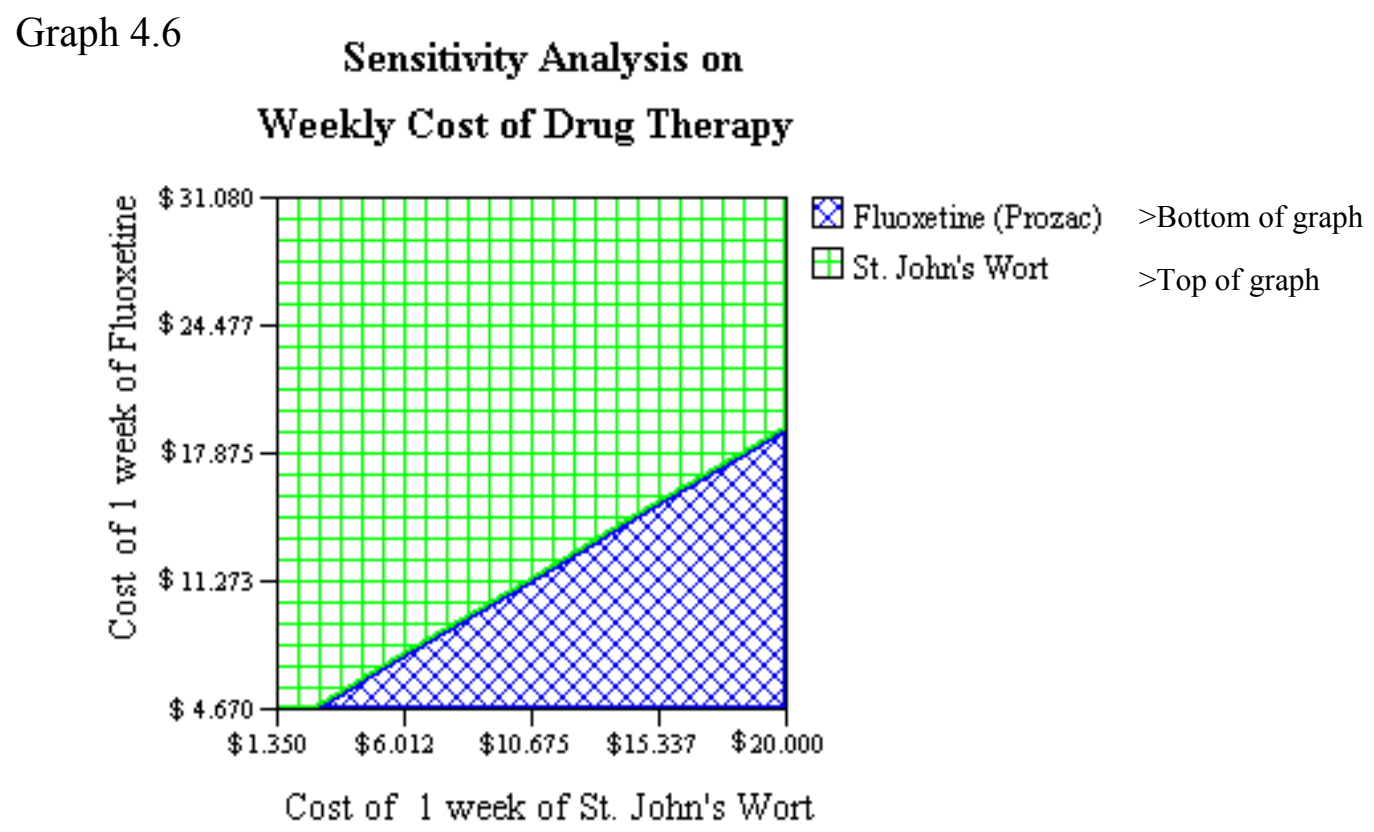

The next variable tested was the probability of remission. First, the value for St. John's Wort was tested over the range of $20 \%$ to $65 \%$ while fluoxetine remained constant at 49\% (Graph 4.7). For all possible values, the outcome did not change. Total expected cost of therapy remained lower for St. John's Wort than fluoxetine. 
Graph 4.7

\section{Sensitivity Analysis on Probability of Remission on St. John's Wort}

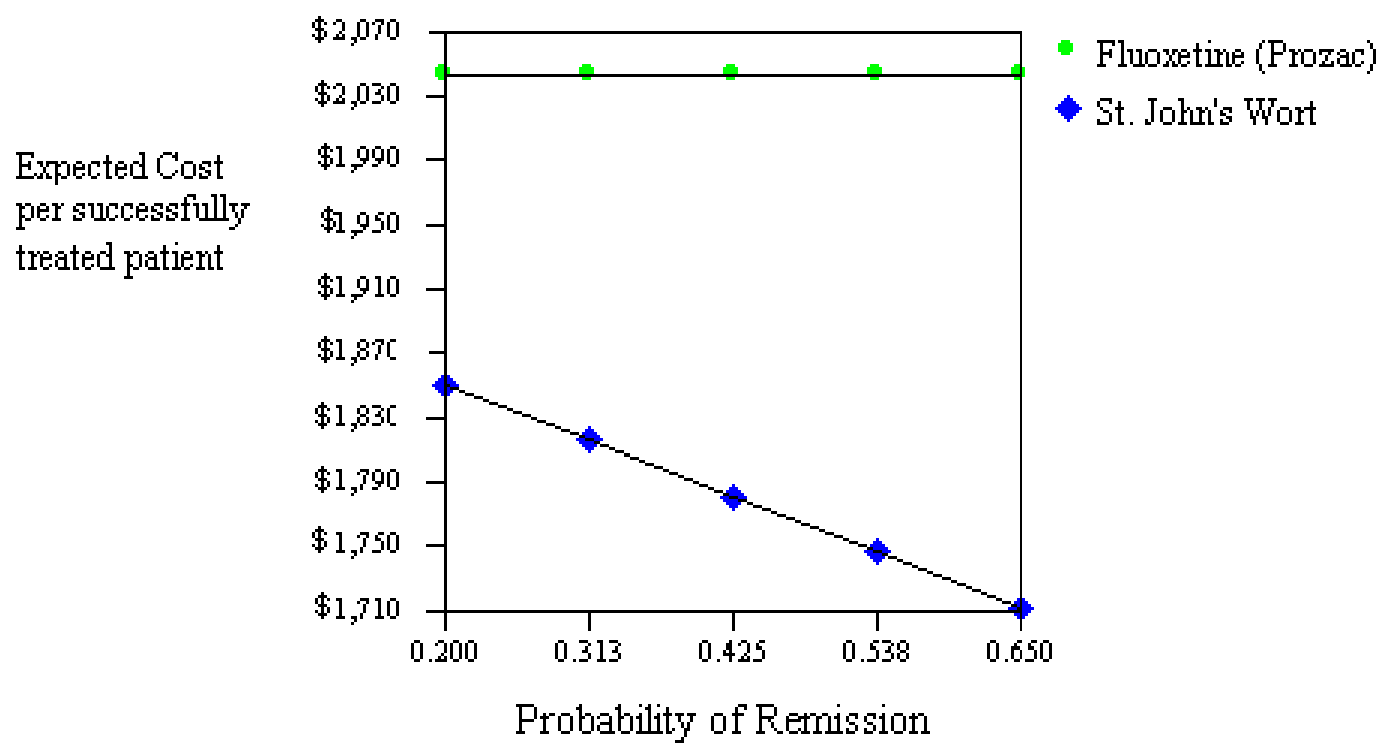

The same analysis was done on the probability of remission for fluoxetine (Graph 4.8). The rate was ranged from $35 \%$ to $90 \%$ while St. John's Wort remained constant at $40 \%$. Again, no difference was detected in this analysis. Although the pathway leading to remission for fluoxetine was the least costly, the rate of remission had no bearing on the overall results. 
Graph 4.8

\section{Sensitivity Analysis on \\ Probability of Remission on Fluoxetine}

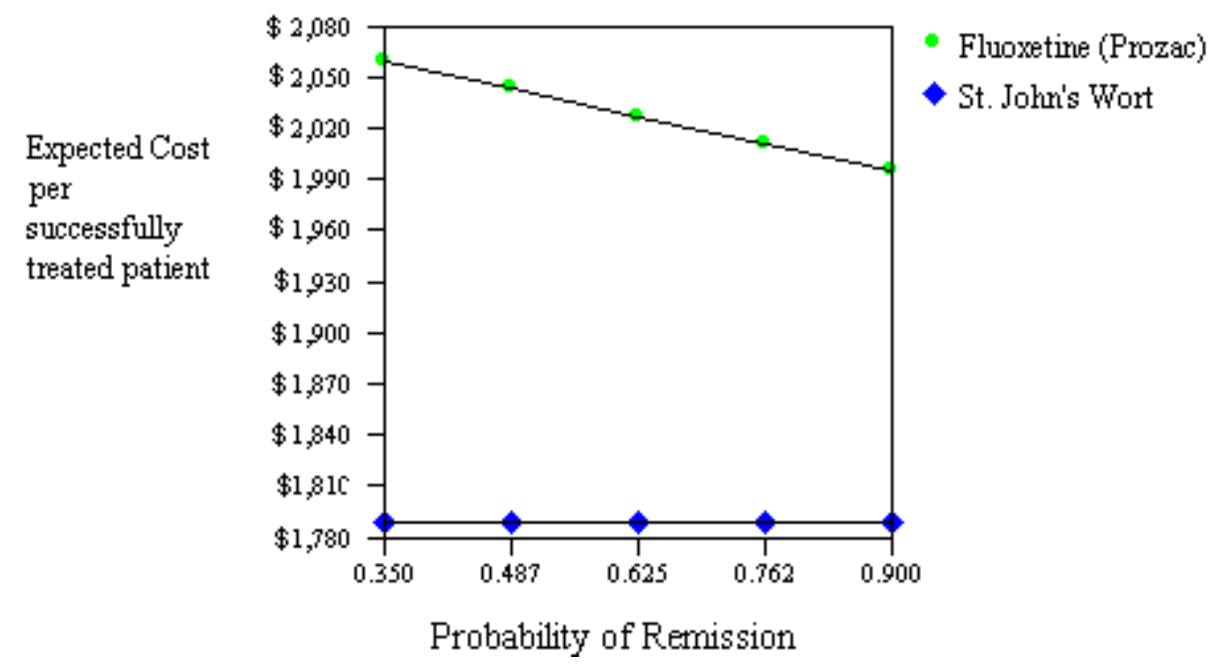

Probability of relapse due to noncompliance was also tested to determine how robust the results were to a change in this variable (Graph 4.9). The rate was varied from $10 \%$ to $85 \%$ for St. John's Wort, and fluoxetine's rate remained constant at 20\%. The expected value of cost per successfully treated patient remained virtually unchanged. For all values in the range, the expected value for St. John's Wort was between $\$ 1,780$ and $\$ 1,800$ per successful treatment. 
Graph 4.9

Sensitivity Analysis on

Probability of Relapse on St. John's Wort

Expected Cost per successfully treated patient

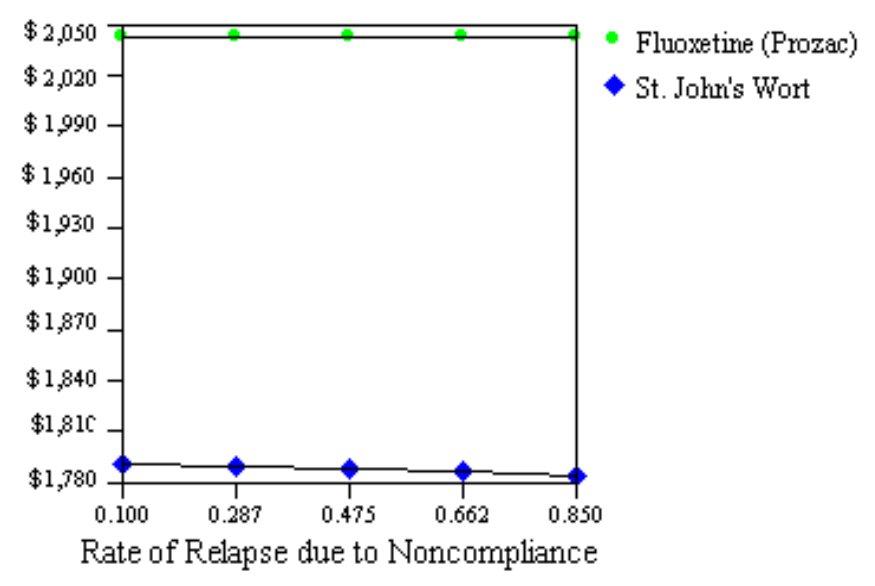

The analysis was repeated for fluoxetine ranging the rate from 5\% to $40 \%$, keeping St. John's Wort at 40\% (Graph 4.10). Again, this resulted in little change to the expected value of fluoxetine. Neither analysis resulted in a change in optimal decision.

Graph 4.10

\section{Sensitivity Analysis on Probability of Relapse on Fluoxetine}

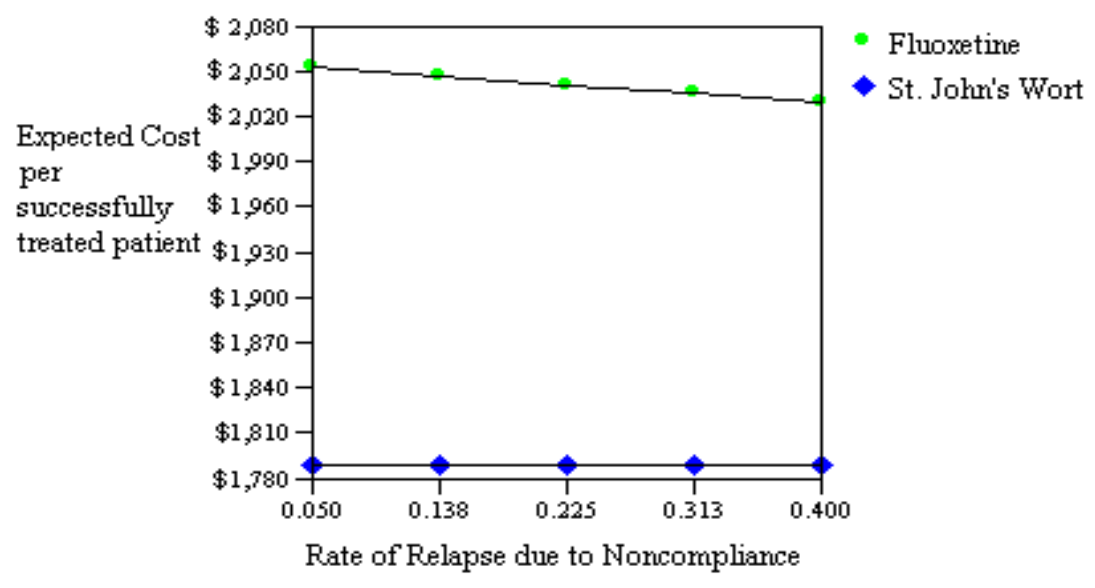


The next variable to be tested was the discontinuation rate for both therapies. St. John's Wort was varied from $2 \%$ to $52 \%$ (Graph 4.11). The high-end was based on four times the maximum rate published in the literature, to try and determine if very liberal estimates would affect the overall result. The low-end represents the discontinuation rate for placebo, estimating that this would be the lowest possible probability. Even at the highest value of 52\%, the expected value for St. John's Wort is well below fluoxetine.

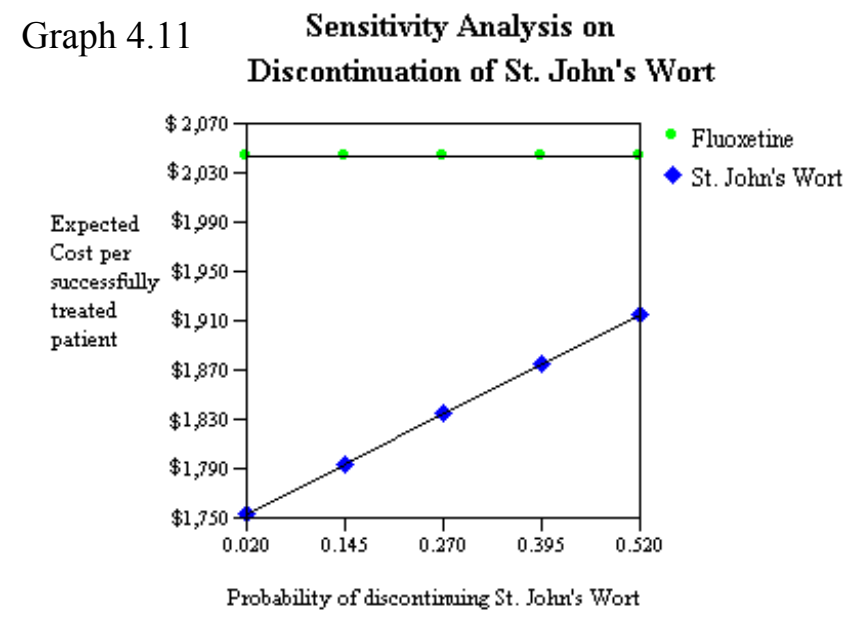

Fluoxetine's discontinuation rate was then varied while keeping St. John's Wort rate constant (Graph 4.12). Fluoxetine was tested over the range of $2 \%$ to $20 \%$. The high end of the range was determined by the expert panel as the frequency of discontinuation secondary to side-effects. Responses for this estimate varied however, with $20 \%$ being the highest and the most frequently reported. The expected value for fluoxetine fluctuated by less than $\$ 10$ over the given range, remaining well above the expected value of St. John's Wort. 
Graph 4.12

\section{Sensitivity Analysis on \\ Discontinuation of Fluoxetine}

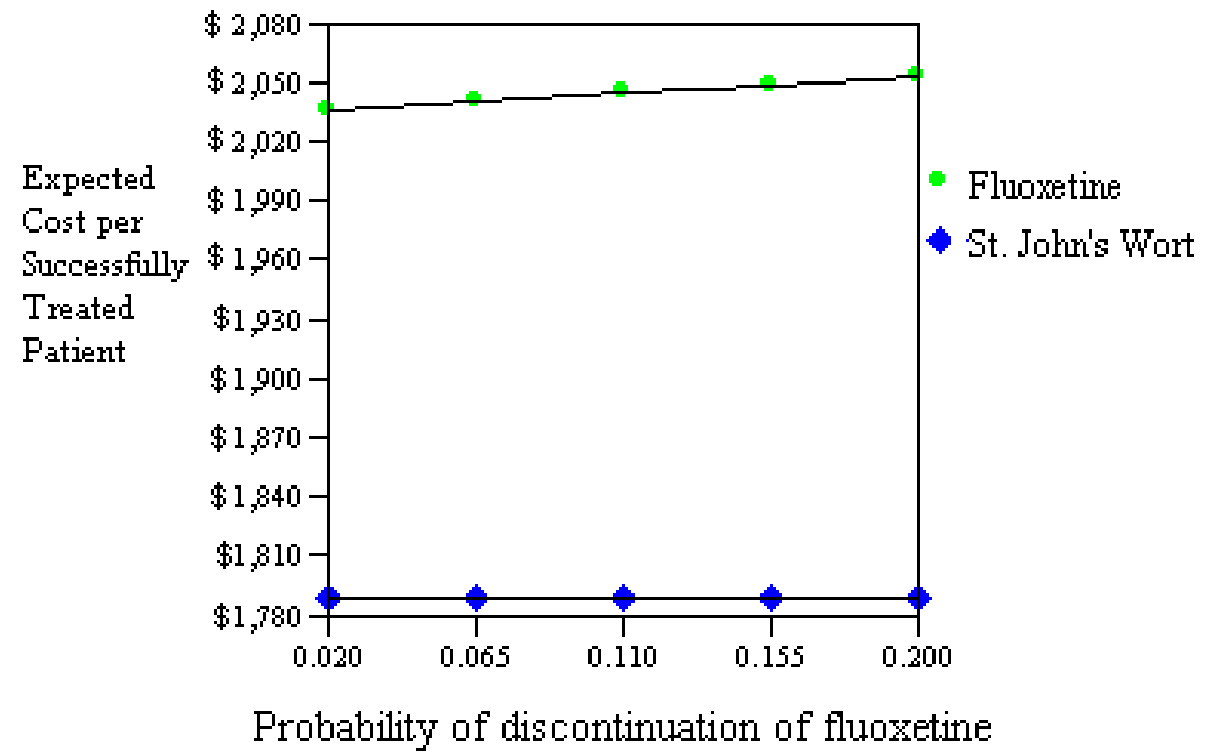

The last variable tested was the probability estimate of the percentage of patients on fluoxetine who would require treatment for the side-effect of agitation. This variable was tested over the range of $2 \%$ to $20 \%$ (Graph 4.13). Cost-effectiveness for fluoxetine changed only slightly over the range of probabilities. Hence, the results were not sensitive to this variable. 
Graph 4.13

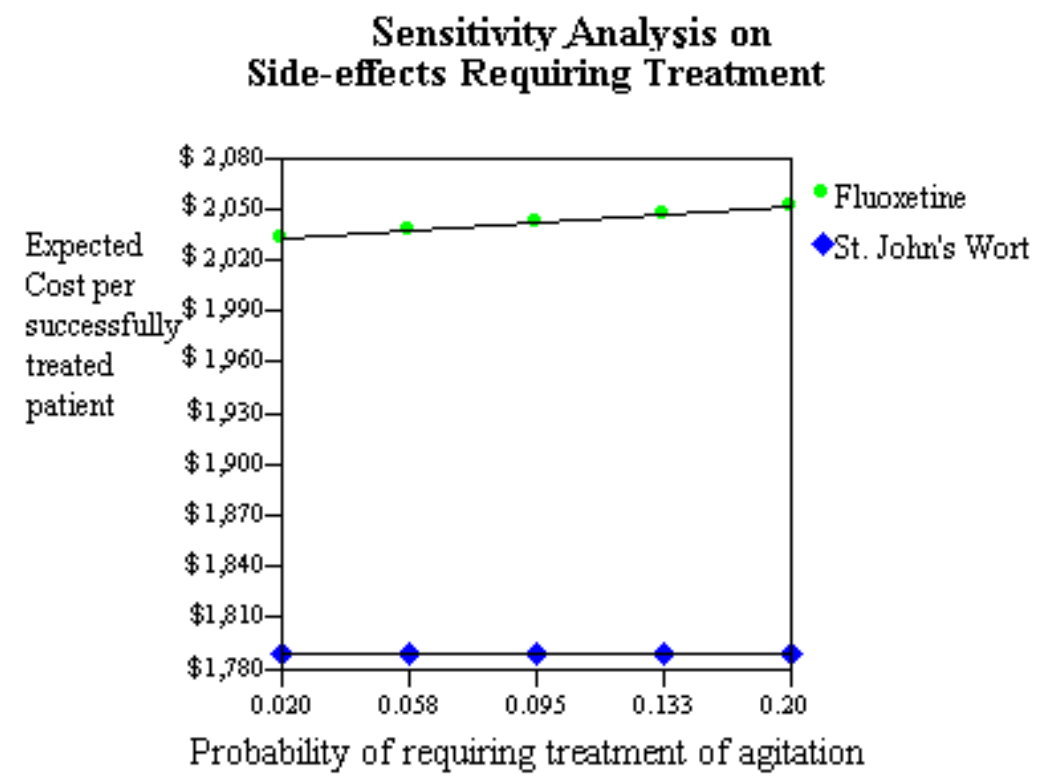

Overall, the results of the base case analysis remained stable and relatively insensitive to changes in most of the variables in the model. Even after the range for response rate was expanded to include the low end of $25 \%$, the results did not change. However, cost of therapy did prove to be an important predictor of the optimal choice of therapy. 


\section{CHAPTER 5 \\ DISCUSSION AND CONCLUSIONS}

\section{INTRODUCTION}

Depression is a debilitating illness that affects approximately $10 \%$ of the American adult population yearly. The devastation it creates is manifested in loss of self-worth and diminished quality of life, loss of productivity, earnings, and in many instances, a loss of social contact. The burden of illness translates into millions of dollars expended on treatment of the social and physical dysfunction that defines the disease.

As healthcare costs continue to skyrocket and financial resources dwindle, both healthcare providers and payers struggle to contain costs while maintaining quality of care. One strategy that many providers of healthcare such as Health Maintenance Organizations (HMO) use as a method of cost-containment is formulary management. This process involves critically evaluating the therapies that are currently marketed and limiting those included on the prescription drug benefit plan to therapies that meet certain criteria.

Ultimately, drugs that deliver a large benefit at a low cost are desired. However, as newer therapies enter the market, they usually come with a high price. Therefore, it is imperative that these therapies be critically evaluated to determine their incremental benefit over current therapies. As such, more companies are turning to economic analyses 
to provide both clinical and economic outcome data to aid in formulary decision-making (Langley, 1996).

St. John's Wort, though not a novel therapy, has within the last several years gained in popularity in the United States as an antidepressant. Through analysis in multiple clinical trials, the herb was demonstrated to be an effective treatment for patients with mild to moderate depression. However, because these evaluations were conducted in rather "sterile" conditions, as required in randomized controlled trials, it is difficult to predict how patients will respond in clinical practice. Additionally, since most of the studies compared the herb to tricyclic antidepressants, more meaningful data would come from comparisons to selective seratonin re-uptake inhibitors (SSRIs), as they continue to dominate the prescription antidepressant market. Because St. John's Wort is relatively inexpensive compared to many of the prescription alternatives, answering these questions provide valuable information to payers of healthcare services.

\section{EXPLANATION OF THE MODEL}

To shed light on this important clinical and economic matter, a cost-effective evaluation was conducted from a payer's perspective using decision analysis to model the outpatient treatment of mild to moderate depression. St. John's Wort was tested against one of the more frequently prescribed antidepressants, fluoxetine, in an effort to maximize external validity. Fluoxetine was chosen as a representative of the class SSRIs. In turn, results of 
the study are more applicable to today's outpatient population as SSRIs continue to be among the top ten most prescribed therapeutic classes (Pink Sheets, 2002).

A decision tree was created based on clinical management of depression as described by a panel of experts experienced in the diagnosis and treatment of depression. This is a common practice in the construction of clinical and financial outcomes of disease state management. The panel consisted of two psychiatrists, one pharmacist who specialized in psychiatry, and three primary care physicians. Although psychiatrists may be thought of as the experts in the treatment of depressive disorders, patients are more often treated by their primary care physicians (Brown, 1997), and therefore family practitioners provide a better reflection of clinical practice.

The practitioners aided in determining and validating the main outcomes of treatment: remission, relapse, and discontinuation of therapy. Associated with those outcomes are the need to treat side-effects, convenience/compliance issues, dosage adjustments, and the need to change drug therapy. As described in the results of the panel survey, there are slight variations in outpatient practice management among practitioners. However, overall management did not vary significantly and closely followed the recommendations of the American Psychiatric Association.

All agreed that SSRIs were the most frequently prescribed agents and were thought to be effective for most patients. Most practitioners agreed that if a patient failed to respond to 
one SSRI, they would switch them to another drug in that class. This practice is supported by literature that shows SSRIs are equally efficacious; hence, choice of therapy depends mainly on the patient's past medical history, chance of adverse effects, preference, potential compliance problems, and financial factors. Because this study was conducted from the perspective of a third party payer with the objective of determining cost-effectiveness of St. John's Wort compared to standard antidepressant therapy, the choice of no pharmacological treatment was not included in the model.

In this study, St. John's Wort was shown to be a more cost-effective therapy than fluoxetine, saving the insurance company approximately $\$ 252$ per patient per year. However, varying the cost of St. John's Wort and/or fluoxetine changes this result. Once fluoxetine's cost drops below $\$ 23.66$ per month, it becomes the more cost-effective choice of therapy with St. John's Wort cost held constant at $\$ 15.77$ per month. Cost estimates were based on published average wholesale prices (AWP). However, this actually represents charges to the third party payer instead of actual cost to the payer. Typically, insurance companies reimburse pharmacies a certain percentage of the AWP plus a dispensing fee. Actual rates depend on the individual providers, the contracts they have with the pharmacies, and the formulary plan provided to members.

The range over which the sensitivity analysis for drug cost was conducted was obtained by using the highest average AWP for brand Prozac ${ }^{\circledR}$ and applying the assumption that 
$25 \%$ of patients require $40 \mathrm{mg}$ while $75 \%$ are maintained on $20 \mathrm{mg}$. This yielded a price per week of $\$ 31.08$ or $\$ 133.11$ for a 30 -day supply.

The average AWP for generic fluoxetine as of Decemember 2002 was $\$ 2.67$ per $20 \mathrm{mg}$ tablet and $\$ 5.32$ per $40 \mathrm{mg}$ tablet. Taking $25 \%$ of the average generic AWP for the lowest dose of fluoxetine $(20 \mathrm{mg})$ yielded the low end of the cost range. This equation was used to estimate reimbursement rates or costs to the third party payers, as opposed to charges. In doing so, the average cost to the payer for a 30-day supply of generic fluoxetine is $\$ 20.03$ or $\$ 4.67$ per week. Thus, even the lowest cost estimate for generic fluoxetine is higher than the breakeven point for cost-effectiveness compared to St. John's Wort as illustrated in Graph 4.6.

Competition among the generic manufacturers is likely to drive the price down for fluoxetine. In other words, as the number of generic manufacturers of fluoxetine increase, the AWP will likely fall, with concurrent diminishes in reimbursement rates. Similarly, the price of St. John's Wort has decreased over the last several years and is expected to continue to fall due to a supply surplus combined with a slight decrease in demand (Ferrier, 2002). As St. John's Wort's price decreases, fluoxetine's cost must get substantially lower for it to be the most cost-effective alternative as demonstrated in Graph 4.6. 
Other than cost of therapy, no other single variable significantly affected the base case results. This may be due to the fact that the primary management of this disease, mild to moderate depression, is drug therapy. Unlike more severe forms of depression that often require hospitalization and frequent physician visits, this disorder is primarily managed in the outpatient setting. Additionally, insurance restrictions typically limit patients' utilization of healthcare resources for mental health. It is not surprising that drug therapy remains the cornerstone of management and therefore, an important predictor of overall cost of treatment.

Response to therapy was initially thought to be a strong predictor of overall costs. The degree of effectiveness of fluoxetine from the clinicians' perspective varied from a $50 \%$ success rate to a $70 \%$ success rate. This coincided closely to the range of $40 \%$ to $77 \%$ published in the medical literature. A one-way sensitivity analysis was conducted over the range of $40 \%$ to $77 \%$ using the maximum and minimum values obtained for fluoxetine. The same was done with the success rate of St. John's Wort, varying it from $43 \%$ to $76 \%$ based on meta-analysis. These analyses demonstrated that the probability of success is not an important determinant of cost-effectiveness in this model.

The National Institute of Mental Health sponsored a \$4 million study conducted at Duke University that assessed the use of St. John's Wort versus sertraline or placebo and focused on the treatment of severe depression (Hypericum Trial Depression Study Group, 2002). The results of this study failed to support use of either agent. Consequently, 
many questions instead of answers arose from this trial. Many speculated that the therapies failed to demonstrate efficacy due to the relatively treatment-resistant population in whom they were tested. The study population had a diagnosis of depression for a minimum of two years.

Several other estimates were not well documented in the literature, and expert opinion varied substantially. The panel estimated the percentage of patients that required treatment with trazodone to be less than $1 \%$ to a high of $20 \%$. This may depend on the characteristics of the patient population each individual practitioner treats. One practitioner may see a disproportionate number of patients with multiple comorbid conditions who may be more sensitive to the drug's adverse effects than otherwise healthy patients. Another reason could stem from some practitioners being more acutely aware of side-effects than others.

In any case, this was one of the possible outcomes identified by the providers and needed to be included in the model. Therefore, estimates were based on randomized clinical trials instead of expert opinion due to the disparity in responses. However, the estimate of $10 \%$ of patients requiring treatment for anxiety may have been greatly underestimated. One study found that $35 \%$ of patients on SSRIs received either an anxiolytic or sedative concurrently based on claims data in a California Medicaid population (Rascati, 1995). Fifteen percent of these prescriptions were for once-daily sedatives implying treatment of sleep disturbances. 
This pathway assumed an additional physician visit would be required to manage the side-effect and that trazodone would be continued for the duration of treatment. Despite this, the sensitivity analysis from this study demonstrated that changing the value of this variable resulted in only minor changes to the expected cost-effectiveness of therapy. Therefore, in this model, treatment of side-effects is not a strong predictor of overall costeffectiveness. This is probably due to the relative low cost of trazodone. In this model, it was estimated to cost approximately $\$ 3.50$ per week or $\$ 15.00$ per month based on average AWP.

Other variables for which there was a lack of information included remission, relapse and compliance rates. Therefore, large ranges were chosen to gauge the effect on the expected cost of therapy. The rate of remission chosen for fluoxetine was based on a trial that studied patients in a natural setting and measured a rate of remission of $49 \%$ at six months. This may seem low based on several other decision analytic models. However, testing its affect over the range of $35 \%$ up to $90 \%$ did not change the results of the study.

Similarly, compliance rates did not seem to affect outcome of cost-effectiveness. Compliance, defined as taking $69 \%$ of the prescribed medication, was extropolated for fluoxetine based on a study comparing compliance rates of different regimens of antihypertensive medications. Once-daily regimens were associated with an $84 \%$ rate of compliance while thrice-daily regimens fell to 59\%. Fluoxetine, though much better tolerated in clinical trials than tricyclics, still has a number of bothersome side-effects 
such as agitation, headache, nausea, sexual dysfunction, and weight gain. Hence, noncompliance was assumed to be $20 \%$ as compared to $16 \%$ found for once-daily regimens. This figure was ranged from $5 \%$ to $40 \%$ but failed to produce significant differences in results. Rates of non-compliance for St. John's Wort were also extrapolated from this study and estimated to be twice that of fluoxetine given its thrice daily regimen, which is similar to the compliance rate of the thrice-daily antihypertensive regimens.

Though St. John's Wort yielded a lower cost per successfully treated patient, the difference was only $\$ 252$ per patient per year. Additionally, as previously mentioned, this is highly dependant on the cost of therapy used in the model. However, it is clear that St. John's Wort is a reasonable alternative to fluoxetine in this study. It may be a wise choice for pharmacy benefit providers to add the herb to their formulary. Although patients have the option of taking the extract on their own given its availability over-thecounter, in many instances they are not under the guidance of a physician and may not take the proper dose. Offering insurance coverage may increase the likelihood that patients will take the herb on the advice of their healthcare provider and will more likely follow the regimen used in clinical trials. It may also lend credibility to the extract in the eyes of patients and healthcare professionals.

The concern over quality and reliability of these herbal products still remain a primary factor among practitioners, patients, and providers alike. Can the products prescribed be trusted to contain the needed ingredients and the quantity required to achieve a clinical 
response? How can one ensure that the product they prescribe will contain exactly what the label claims? The American College of Physicians (ACP) and American Society of Internal Medicine (ASIM) guidelines include the statement that St. John's Wort is not approved by the Food and Drug Administration (FDA) and warn that products may vary from those tested in clinical trials.

Despite the results of several randomized clinical trials and the results of this study, many practitioners may still be unwilling to "prescribe" a non-FDA approved herbal. Although it is common for patients to self-medicate with herbals without their doctor's knowledge, let alone their recommendation, prescribers may be concerned with legal ramifications of prescribing these products, should an adverse event occur. In contrast, prescribers often use medications for non-FDA approved indications when strong clinical evidence exists. Many pharmaceutical manufacturers are aware of this and forego the costly process of gaining FDA approval. One example is the use of aspirin for prevention of myocardial infarction in high-risk patients. Based on extensive literature, prescribing aspirin for this indication has become standard of therapy. However, it is not a FDA-approved indication. In this regard, herbal manufacturers are unlikely to pursue FDA approval since consumers are already using their products.

Hyperforin is generally recognized as the primary active constituent of St. John's Wort. However, hyperforin is very unstable and rapidly degrades in the presence of light or oxygen. Therefore, it is rarely used to standardize products. Rather, hypericin is 
commonly used for standardization. Valid assays have been developed to accurately determine hypericin and hyperforin content without compromising integrity using highperformance liquid chromatography. This method was used to assess the content of several brands of Hypericum (de los Reyes, 2002). Some of the products were found to have either lower or higher amounts of hypericins than claimed on the label. This also has lead to hesitation in using or recommending use of this as well as other nutraceuticals.

Product quality is dependent on a number of different factors such as crop variability due to growing conditions, contamination, and manufacturing practices. In vitro culture technology has been developed to solve this problem of standardization. The technique allows for production in a sterile, standardized condition ensuring products are consistent and constituents are precise, though it may be cost prohibitive at this stage. There is currently considerable variability among the products marketed in terms of constituent amount and standardardization practice. Consequently, it is recommended that the FDA through the Diet Safety Health and Education Act, require more stringent regulation on the manufacturing of dietary supplements. Standardization protocols including specific assays should be required to ensure quality. Strict regulatory controls on manufacturing practices, initiated in 1994, are still years from being implemented. Until this is law, one proposal is to use the brands that were tested in the clinical trials. 
One brand in particular, LI 160, manufactured by Lichter Pharmaceuticals in Germany and marketed under the brand name Kira (Jarsin in Germany), has been tested in numerous clinical trials. It is however, one of the more expensive brands found on the market. It retails at $\$ 20.58$ for a 30 -day supply, which is still substantially lower than fluoxetine's average retail price. Nonetheless, this product provides some assurance that the actual contents match the label.

\section{STRENGTHS OF THE STUDY}

There are currently no published data regarding the cost-effectiveness of St. John's Wort. However, this study has provided information to third party payers to aid in formulary management strategies. By employing the method of decision analysis, insurance providers can make more informed decisions under conditions of uncertainty. Decision analysis allows one to understand the impact that uncertainty has on the final result, in this case, cost-effectiveness of the drug.

Unlike a randomized controlled trial, decision analysis can be more reflective of realworld conditions by amassing clinical judgement and clinical trial results to portray the likely outcome of using St. John's Wort over fluoxetine. For example, in randomized controlled trials involving fluoxetine, patients are randomized to active treatment, usually consisting of fluoxetine given in a dose of $20 \mathrm{mg}$ once daily. Dosage adjustments are rarely, if ever, allowed in the protocol. However, as described by the expert panel, it is common to increase the dose of fluoxetine if an adequate response is not achieved. The 
model in this study incorporated dosage increases in the cost of therapy and modeled the treatment of depression based on clinical practice as described by the providers of care. Additionally, according to previous studies, depression is often managed differently than what has been recommended. Frequently, patients are prescribed inappropriate doses and/or for inadequate durations of time (Ormel, 1991;Katon et al, 1992). Therefore, it was important to use information obtained directly from the healthcare practitioners that most often provide care to these patients, instead of relying strictly on consensus guidelines.

This study has provided a model for healthcare providers and payers to utilize in making policy and individual patient decisions. Providing estimates and ranges along with the sources of data used allows decision-makers to determine the relevance of the results to their patient or customer population. This is referred to as the "transparency" of the model. Additionally, the sensitivity analyses conducted test the robustness of the data and better explain the impact various inputs have on the final result.

Another strength of the study is that it provided information on the cost-effectiveness of St. John's Wort. Prospective studies such as pragmatic or naturalistic trials provide excellent data in "real-world" conditions and are therefore generalizable to the population likely to be affected. On the other hand, they are usually very costly and time consuming. Given the rising costs of pharmaceuticals and the grave need to control 
spending, healthcare decision-makers need information quickly and most likely do not have the resources to conduct such evaluations.

\section{ASSUMPTIONS AND LIMITATIONS}

The analysis was conducted based on a number of assumptions. Patients failing to respond to initial treatment (either from discontinuation, relapse, or initial failure) were assumed to respond to the second course of therapy and therefore, the full cost of the second drug therapy was incurred along with the associated number of physician and therapist visits. In real world conditions, however, patients go through the same cycle of having a probability of responding to or failing the secondary therapy. To simplify the model, this probability was set at $100 \%$. Therefore, total cost of therapy for both arms of the decision tree may be underestimated. However, since the objective of the study was to measure the overall difference in cost-effectiveness, precise cost estimates of variables such as physician visits are not crucial if the same estimates are used in both treatment arms of the decision tree.

The model assumes that all treatment failures are switched to paroxetine (Paxil $\left.{ }^{\circledR}\right)$ except for patients initially treated with fluoxetine who relapse due to noncompliance. These patients would continue another six months of fluoxetine given that it is a once daily regimen and paroxetine would offer no advantage in this respect. Treatment failure or relapse also implies the number of physician visits increases from six to seven per year. Similarly, psychotherapy visits increase from five to six per year. The reason for this is 
that it is presumed that patients in a state of depression (failure to respond to therapy) are more likely to utilize healthcare services than those who are being successfully treated by medications.

Psychotherapy was included as an important component of the treatment model. This was based upon responses from the expert panel stating that most would refer a patient to a therapist in addition to prescribing drug therapy. It is important to note that the panel members were all from an urban university setting where there is access to trained psycologists and psychiatrists. However, in more rural settings, access is greatly limited. Therefore, the cost of psycotherapy visits would not apply and overall cost of care may be reduced. On the other hand, response rates may decrease and relapse rates may increase resulting in an overall increase in cost of care. In any case, overall results are difficult to predict.

Treatment for side-effects due to fluoxetine was limited to trazodone. This was described as the most frequently prescribed medication for treatment-related side-effects due to fluoxetine. However, it is possible that other medications may be required, though they were not represented in this model. Patients on St. John's Wort were assumed not to require any additional medical treatment for adverse effects. Side-effects from the herb were assumed to be amenable to over-the-counter medications for which third-party providers would not bare the costs. This seems reasonable since large observational 
studies on St. John's Wort have failed to demonstrate any significant side-effects requiring treatment.

Direct costs of medical care were based on charges obtained from physician offices in Connecticut. These costs may not be representative of other areas of the country, nor are they representative of the public health sector. Though total cost of care may be over or underestimated, as pointed out before, these costs did not differ between therapies and therefore will not affect choice of therapy based on incremental cost-effectiveness. On the other hand, cost of therapy, does make a significant difference on the results. Acquisition cost for each therapy was based on average wholesale price (AWP) or prices obtained from retailers located in southeastern Connecticut. These prices may differ significantly from those found outside of Connecticut. The model also did not include inpatient cost of care. It was assumed that patients with mild to moderate depression would not require hospitalization for care of the illness.

It is important to point out that the survey of the expert panel was conducted in 1998 when fluoxetine was only available as the brand product, Prozac $\AA$. Responses may vary somewhat today based on its availability as a generic product. Factors such as patients' and practitioners' preference for brand products may play a role in prescribing the drug and how effective it is perceived to be. Additionally, the panel had no experience in prescribing St. John's Wort. Only a few of them had any prior knowledge of the herb. Therefore, all estimates of effectiveness were culled from the literature. 
In many circumstances, estimates were based on short-term trials (six to eight weeks) and extrapolated to the one-year cycle of the model. Discontinuation rates, for instance, were obtained from six-week trials, but rates of discontinuation differ over time. Multiple studies were needed to find all the estimates required in the model, representing a rather heterogeneous population. In one way, this aids in generalizability; however internal validity is diminished. Separately, the studies tested only individuals with mild to moderate depression. Therefore, results can only be generalized to this population.

Analysis of the study was limited to a period of one year. Therefore, subsequent relapses that may occur are not accounted for in this model. However, this may be less relevant to third party payers due to possible attrition of members. This is one reason many insurance providers don't cover preventative therapy. The company doesn't retain the member long enough to reap the future benefits of decreased cost of care. Therefore, cost-effectiveness of one year of therapy may be more practical in terms of making policy decisions.

The perspective of this study was that of a third party payer, such as a health maintenance organization (HMO). Costs such as out-of-pocket expenses and outcomes such as lost wages or productivity were not accounted for in the model as they would not be incurred directly by the HMO. In general, it has been recommended to conduct cost-effective analyses from the societal perspective. However, as information is not always readily 
available, this can be an arduous task. Additionally, results of such a trial may be difficult to interpret for the purposes of this study.

\section{SIGNIFICANCE OF THE STUDY}

Depression is a devastating illness that has significantly impacted the public both economically and physically. There is a great need to critically evaluate the potential of any new therapy that may prove to be of benefit. St. John's Wort became increasingly popular in the United States as a mood stabilizer in the mid-1990s. Although it is still among the top selling herbal products, the fervor has calmed somewhat since then. This may be due to media attention in the past year questioning its use in depression or just representative of the overall decline in the economy. Add to this the lack of regulation on advertising and marketing of herbal products and it underscores the importance of critical evaluation of both cost and clinical outcomes.

It is clear the public needs an unbiased assessment of these products evaluated against the standard of care. This study serves that purpose. It provides relevant information to patients and providers of healthcare in helping to better allocate limited resources. The analysis serves as an integration of expert opinion and clinical data into a model of current clinical practice. The decision tree developed may also be used as a model to assess future treatment modalities. 


\section{CONCLUSIONS}

It is evident that St. John's Wort represents a viable alternative to fluoxetine and most likely to other prescription antidepressants based on the wealth of clinical information available and supporting data on its cost-effectiveness from this study. The Agency for Health Care Policy and Research recognizes St. John's Wort as a rationale therapy in the treatment of mild depressive disorder. The ACP and ASIM guidelines also include St. John's Wort as a treatment option for short-term treatment of mild acute depression. Given this, healthcare providers should strongly consider adding it to the armamentarium of drugs included in their prescription drug benefit plan.

A variety of drugs should be available to patients and healthcare practitioners in order to tailor therapy based on individual circumstances such as tolerability, compliance, comorbid illnesses, possible drug interactions, and allergies. By offering St. John's Wort on drug benefit plans, third party payers may realize long-term cost savings. Initial costs may increase if patients who would normally not seek medical attention begin to utilize medical services in order to get insurance coverage for the herb. However, in the long run, the increase in medical utilization may actually decrease overall costs of care. If patients with more mild forms of the disease are treated under the care of a physician and take the product according to recommendations, then potentially more patients will be adequately treated and ultimately use fewer healthcare resources. 
The overall difference in savings in this study is not dramatic, but it is consistent with what other studies comparing antidepressant therapies have found. Adding St. John's Wort to a drug formulary will offer patients a safe and effective alternative to prescription products. This addition may not offer huge financial savings short-term, but if utilized may help calm the rate of spending the healthcare system has experienced. However, until the FDA has tighter controls on the manufacturing of St. John's Wort products or a prescription status product becomes available, prescribers may still tend to shy away from prescribing this herbal remedy, regardless of cost-effectiveness.

\section{FUTURE RESEARCH}

There is a need for additional studies such as this one to be conducted. As mentioned previously, it is generally recommended to conduct economic evaluations from the perspective of society to capture all costs and consequences of the treatment choice. This, of course, would require an intense amount of resources in terms of both time and money. Although this is no easy task, assessing quality of life parameters may be quite possible. Obtaining utility values would enable one to conduct cost-utility analyses that could be done from either the patient or the payers perspective. It would be of great use to have information regarding patients' quality of life on this herbal remedy compared to standard treatments such as the SSRIs. Additionally, studies that focus on newer treatments like mirtazapine $\left(\right.$ Remeron $\left.{ }^{\circledR}\right)$ would also be of great benefit. 
Long-term studies on St. John's Wort are still needed to determine efficacy past the acute phase of treatment. This would be best accomplished through a randomized controlled trial that tested both placebo and another standard antidepressant. Effectiveness trials are also warranted to better understand how use of the herb in clinical practice affects outcomes of cost and quality. 


\section{REFERENCES}

Aguglia, E and M. Casacchia et al. Double-blind study of the efficacy and safety of sertarline versus fluoxetine in major depression. Internals of Clinical

Psychopharmacology 1993; 10: 393-405.

AHCPR Publication No. 99-E014. Evidence Report/Technology Assessment Number 7 Treatment of Depression: Newer Pharmacotherapies. Prepared for: Agency for Health Care Policy and Research, U.S. Department of Health and Human Services 2101 East Jefferson Street Rockville, MD 20852 February 1999.

American Psychiatric Association. Diagnstic and Statistical Manual for Mental Disorders, Fourth Edition. (DSM -IV). Washington, DC: American Psychiatric Press, 1993.

American Psychiatric Association. Handbook of Psychiatric Measures. American Psychiatric Press, Arlington, VA. Jul, 2000; 1: 455.

Associated Press, New York. Herb grows popular after drug ban-worts and all. Dominion Post. October 18, 1997: 10-B.

Astin, JA. Why patients use alternative medicine: results of a national study. JAMA 1998; 279(19): 1548-1553.

Baladi JF. Selective serotonin reuptake inhibitors (SSRIS) for major depression. Part II: The cost-effectiveness of SSRIS. Ottawa: Canadian Coordinating Office for Health Technology Assessment (CCOHTA); 1997.

Batz, Forrest. Integrating Herbal Therapy Into Practice. American Druggist May 1998: 58-63.

Bech, P., M. Kastrup, O.J. Rafaelsen. Mini-compendium of Rating Scales for Anxiety,Depression, Mania, Schizophrenia with Corresponding DSM-III Syndromes. ACTA Psychiatry Scandinavia 1986, 73:1-39.

Behnke, Kirsten, Jensen, G., Graubaum, H., and Gruenwald, J. Hypericum perforatum versus Fluoxetine in the Treatment of Mild to Moderate Depression. Advances In Therapy 2002; 19 (1):43-52.

Benedict, Carey. The Sunshine Supplement. Health January 1998; 12: 52-55.

Benefield, William. Cost/Benefits in Managing Depression. Behavioral Health Management November/December, 1995; 15 (6): 12-17. 
Bilia, Anna Rita and Sandra Gallori and Franco F. Vincieri. St. John's Wort and depression. Efficacy, safety, and tolerability-an Update. Life Sciences. 2002; 70: 30773096.

Bischoff, RC, Bobon, D, Görtelmeyer, R, Horn, R, Müller, A, Stoll, Woggon, B. Rating Scales for Psychiatry,CIPs, European Edition Beltz Verlag, Weinheim, 1990

Blackwell, B. The drug regimen and treatment compliance. In: Taylor, $D W$ and $D L$ Sackett. eds. Compliance in Health Care. Baltimore, Md: Johns Hopkins University Press, 1979.

Bloomfield, H. Treating Depression With Hypericum. Saturday Evening Post November, 1997; 269: 62-5.

Bloomfield, H, McWilliams, M. Hypericum \& Depression: Can Depression Be Successfully Treated with a Safe, Inexpensive, Medically Proven Herb Available without a Prescription? Prelude Press; Los Angeles, 1996.

Bombardelli, Ezio and Paolo Morazzoni. Hypericum Perforatum. Fitoterapia. 1995; 66: 43-67.

Bootman, J. Lyle, Townsend, Faymond J., McGhan, William F. Introduction to Pharmacoeconomics In Principles of Pharmacoeconomics $2^{\text {nd }}$ edition Harvey Whitney Books Company 1996.

Brenner, R, Madhusoodanan, S, Pawlowska M, Czobor, P. St. John's wort and major depression. [Letter] JAMA 2001; 286(1): 43.

Brevoort P. The Booming US Botanical Market. Herbalgram 1998; 44: 33-46.

Brink, S. Singing The Prozac Blues. US News and World Report. November, 1993; 115: 76-80.

Brown, D. et al. What You Need to Know About Psychatric Drugs. "Depression: Clinical Applications of Natural Medicine." 1997; 35.

Chavez, M and Pedro Chavez. Saint John's Wort. Hospital Pharmacy 1997; 32 (12): $1621-1632$.

Cooperman, T and William Obermeyer. Do All Suplements Contain What They Say They Contain? US Pharmacist October 2002; 27:10.

Cott, J. Medicinal Plants and Dietary Supplements. Sources for Innovative Treatment or Adjuncts? Psychopharmacology Bulletin 1995; 31: 131-137.

Cott, JM, Rosenthal, N, Blumenthal, M. St. John's wort and major depression. [Letter] JAMA 2001; 286(1): 44-45. 
Colchamiro, R. Worting Away Depression. American Druggist January, 1998: 29-32.

Cristser, G. Oh, How Happy We Will Be. Harper's Magazine June, 1996; 292 (1753): 39-49.

de los Reyes GC, Koda RT. Determining hyperforin and hypericin content in eight brands of St. John's wort. American Journal of Health-System Pharmacists 2002;59:545-7.

Depression Guideline Panel. Depression in Primary Care: Detection, Diagnosis, and Treatment. Quick Reference Guide for Clinicians, Number 5. Rockville, MD. U.S. Department of Health and Human Services, Public Health Service, Agency for Health Care Policy and Research. AHCPR Publication No. 93-0552. April 1993.

Depression Guideline Panel. Depression in Primary Care: Volume 1. Detection and Diagnosis. Clinical Practice Guideline, Number 5. Rockville, MD. U.S. Department of Health and Human Service, Agency for Health Care Policy and Research. AHCPR Publication No. 93-0550. April 1993.

De Smet, P and Willem Nolen. St. John's Wort as an antidepressant. BMJ August, 1996; 313:276-278.

Drummond, M, O'Brien, Bernie, Stoddart, Greg L., Torrance, George W. Methods for the Economic Evaluation of Health Care Programmes $2^{\text {nd }}$ edition Oxford: Oxford Medical Publications, 1997.

Eddy, DM. Clinical Decision Making: from theory to practice. Cost-effectiveness analysis. Is it up to the task? JAMA 1992; 267(24): 3342-3348.

Edwards JG. Prevention of relapse and recurrence of depression: newer versus older antidepressants. Advanced Psych Treatment 1997; 3 52-7

Eisen, SA, Miller, DK, Woodward, RS, Spitznagel E., Przybeck TR. The effect of prescribed daily dose frequency on patient medication compliance. Archives of Internal Medicine 1990; 150(9): 1881-1884.

Eisenberg D, Davis R, Ettner S, Appel S, Wilkey S Van Rompay M, Kessler R. Trends in alternative medicine use in the United States, 1990-1997. Results of a follow-up national survey. JAMA 1998; 280:1569-75.

Einarson, T, Arikian, Steve, Sweeney, Susan, and Doyle, John. A Model to Evaluate the Cost-Effectiveness of Oral Therapies in the Management of Patients with Major Depressive Disorders. Clinical Therapeutics 1995; 17(1): 136-153.

Ernst, E. Second thoughts about safety of St. John's wort. Lancet 1999; 354: 2014-6. 
Ernst, E. St. Johns's Wort, an anti-depressant? A systematic, criteria-based review. Phytomedicine 1995; 2 (1): 67-71.

Escitalopram (Lexapro) for Depression. The Medical Letter. September 30, 2002; 44(1140): 83 .

Fawcett J, Barkin RL. Efficacy issues with antidepressants. Journal of Clinical Psychiatry. 1997;58:32-39.

Federal Guidelines: Depression Often Treatable In Primary Care. Geriatrics July, 1993; 48 (7): 21.

Ferrier, G. US Herbal Market At A Crossroads. Functional Foods \& Nutraceuticals March, 2002. www.newhope.com/ffn/ffn_backs/mar_02/herbmarket.cfm

Fink, A and Jacqueline Kosecoff, Mark Chassin, Robert H. Brook. Consensus Methods: Characteristics and Guidelines for Use. American Journal of Public Health 1984; 74: 979-84.

Fisherman, R. Treating Depression. Lancet November, 1994; 44 (8932): 1291.

Frank, L, Revicki, Dennis A., Sorensen, Sonja V., and Shih, Ya-Chen Tina. The Economics of Selective Serotonin Reuptake Inhibitors In Depression: A Critical Review. CNS Drugs 2001; 15(1): 59-83.

Fugh-Berman, A. St John's wort and major depression. [Letter] JAMA 2001; 286(1): 43-5.

Gagiano, CA. A double-blind comparison of paroxetine and fluoxetine in patients with major depression. British Journal of Clinical Research. 1993; 4: 145-152.

Gargiulo, M. Potency of Saint-John's-Wort Often Different than Label. http://homearts.com/depts/health/98news21.html.

Gaster, B and John Holroyd. St. John's Wort for Depression. A Systematic Review. Archives of Internal Medicine 2000; 160: 152-156.

Greenberg PE et al. Depression: a neglected major illness. Journal Clinical Psychiatry 1993;54: 419-424

Halama, P. Wirksamkeit des Hypericum Extraktes LI 160 bei 50 Patienteneiner psychiatrischen Fachpraxis. Nervenheilkunde 1991; 10:305-307.

Hamilton, M. Rating Scale for Depression. Journal of Neurology, Neurosurgery and Psychiatry 1960, 23:56-61. 
Hansgen, KD and J Vesper, M Ploch. Multicenter Double-Blind Study Examining the Antidepressant Effectiveness of the Hypericum Extract LI 160. Journal of Geriatric Psychiatry and Neurology October, 1994; 7 (supplement 1): 15-18.

Harrer, G, Hubner, WD, Podzuweit, H. Effectiveness and Tolerance of the Hypericum Extract LI 160 Compared to Maprotiline: A Multicenter Double-Blind Study. Journal Geriatric Psychiatry Neurology 1994; 7(suppl 1): S24-S28.

Harrer G and Sommer, H. Placebo-Controlled Double-Blind Study Examining the Effectiveness of an Hypericum Preparation in 105 Mildly Depressed Patients. Journal of Geriatric Psychiatry and Neurology. October, 1994; 7 (supplement 1): 9-12.

Harrer, G, Schmidt, U, Kuhn, U., Biller, A. Comparison of equivalence between St. John's wort extract LoHyp-57 and fluoxetine. Arzneimittel-Forschung 1999; 49(4): 289296.

Harrer, G, Schmidt, U, Kuhn, U. Alternative Depressionsbehandlung mit einem Hypericum-Extrakt. Neurolologie Psychiatrie 1991; 5: 710-716.

Harrison, P. Herbal Medicine Takes Root in Germany. Canadian Medical Association Journal. March, 1998; 158: 637-641.

Hawley, C. St. John's wort was not better than placebo for reducing depression scores. (Commentary) Evidence Based Medicine 2001; 6: 185.

Henry, J and Carol Rivas. Constraints on Antidepressant Prescribing and Princples of Cost-Effective Antidepressant Use. Pharmacoeconomics June, 1997; 11(6): 515-537.

Hill, D. Take a Pill! Brandweek December, 1997; 38: 22-25.

Hobbs, C. St. John's Wort: Hypericum perforatum L: A review. HerbalGram 1989; 18/19: 24-33.

Hubner, WD and S Lande, H Podzuweit. Hypericum Treatment of Mild Depressions with Somatic Symptoms. Journal of Geriatric Psychiatry and Neurology October, 1993; 7 (supplement 1): 12-14.

Huff, C. Nature’s Prozac. Remedy March/April, 1998: 8-10.

Hypericum Depression Trial Study Group. Effect of hypericum perforatum in major depressive disorder: a randomized controlled trial. JAMA 2002; 287(14): 1853-1854.

Jonsson B., Bebbington PE. What price depression? The cost of depression and the costeffectiveness of pharmacological treatment. British Journal of Psychiatry 1994; 164(5):665-73. 
Johnson, D and H Ksciuk, H Woelk, E Sauerwein-Giese, A Frauendorf. Effects of Hypericum Extract LI 160 Compared with Maprotiline on Resting EEG and Evoked Potentials in 24 Volunteers. Journal of Geriatric Psychiatry and Neurology October, 1994; 7 (Supplement 1): 44-46.

Kalb, R. and RD Trautmann-Sponse, and M. Kieser. Efficacy and Tolerability of Hypericum Extract WS 5572 versus Placebo in Mildly to Moderately Depressed Patients. Pharmacopsychiatry 2001; 34: 96-103.

Katon W, Von Korff M, Lin E, Bush T, Ormel J. Adequacy and duration of antidepressant treatment in primary care. Medical Care 1992; 30:67-76.

Kessler, RC and S Zhao, DG Blazer, M Swartz. Prevalence, Correlates, and Course of Minor Depression and Major Depression in the National Comorbidity Survey. Journal of Affective Disorders August, 1997; 45 (1-2): 19-30.

Kim, Hannah, Jon Streltzer, and Deborah Goebert. St. John's Wort for Depression: A Meta-Anaysis of Well-Defined Clinical Trials. The Journal of Nervous and Mental Diseases September 1999; 187(9): 532-538.

King, J. The American Dispensatory $7^{\text {th }}$ edition Cincinnati: Moore, Wilstach \& Baldwin; 1866.

Kroenke, K, et al. Similar Effectiveness of Paroxetine, Fluoxetine, and Sertraline in Primary Care. A Randomized Trial. JAMA 2001; 286(23): 2947-2955.

Langley PC, Sullivan SD. Pharmacoeconomic evaluations guidelines: guidelines for drug purchasers. Journal of Managed Care Pharmacy 1996; 2:671-7.

Lapierre, Y, Bentkover, J. Direct cost of depression. Canadian Journal of Psychiatry 1995; 40 (7): 370-377.

Lawler, F. Clinical Use of Decision Analysis. Medical Decision Making. June, 1995; 22 (2): 281-293.

Lecrubier, Y. et. al. Efficacy of St. John's Wort Extract WS 5570 in Major Depression: A Double-Blind, Placebo-Controlled Trial. American Journal of Psychiatry 2002;159: 1361-1366.

Linde, K and Gilbert Ramirez, Cynthia D Mulrow, Andrej Pauls, Wolfgang Weidenhammer, Dieter Melchart. St. John's Wort for Depression-An Overview and Meta-Analysis of Randomised Clinical Trials. BMJ August, 1996; 313: 253-258. 
Lovibond, PF., and S.H. Lovibond. The Structure of Negative Emotional States: Comparison of the Depression Anxiety Stress (DASS) with the Beck Depression and Anxiety Inventories. Behavioral Research and Therapy 1995, 33:335-343.

Luce, B R., Simpson, Kit. Methods of Cost-Effectiveness Analysis: Areas of Consensus and Debate. Clinical Therapeutics 1995; 17(1): 109-125.

Lusted, LB. Decision making in patient management. NEJM 1971; 284:415-424.

Maier, W et al. Improving Depression Severity Assessment- II. Content,Concurrent and External Validity of Three Observer Depression Scales. Journal of Psychiatric Research 1988, 22:3-12.

Martinez, B and S Kasper, S Ruhrmann, HJ Moller. Hypericum in the Treatment of Seasonal Affective Disorders. Journal of Geriatric Psychiatry and Neurology October, 1994; 7 (supplement 1): 29-33.

Meek, PD. "Herbal Remedy Use and Case Reports of Associated Toxicity." Pharmacist Letter reference \# 121016: p 1-3.

Miller, Alan. St. John's Wort (Hypericum perforatum): Clinical Effects on Depression and Other Conditions. Alternative Medicine Review. 1998; 3 (1): 18-26.

Moller, H and Hans-Peter Volz. Drug Treatment of Depression in the 1990s: An Overview of Achievements and Future Possibilities. Drugs. November 1996; 52 (5): $625-638$

Muller HJ, Volz HP. Drug treatment of depression in 1990s. An overview of achievements and future possibilities. Drugs 1996; 52(5): 625-638.

Muller WE, Rolli M, Schafer C, Hafner U. Effects of Hypericum extract in biochemical models of antidepressant activity. Pharmacopsychiatry 1997; 30(2):102-107.

Murray, MT. Lifestyles and dietary factors in depression. American Journal of Natural Medicine December, 1995; 2 (10): 10-15.

Murray, M. St. John's Wort In Depression. Abstracts and Commentary. The American Journal of Natural Medicine. 1995; 25.

Murray, C, JL, Lopez, Alan D. The Global Burden of Disease: A comprehensive Assessment of Mortality and Disability from Diseases, Injuries, and Risk factors in 1990 and Projected to 2020. Boston, Harvard School of Public Health on behalf of WHO and World Bank, 1996.

Murray, CJ, Lopez AD. Evidence-based health policy-lessons from the Global Burden of Disease Study. Science 1996; 274: 740-3. 
Narrow WE. One year prevalence of depressive disorders among adults 18 and over in the US: NIMH ECA prospective data. Population estimates based on U.S. Census estimated residential population age 18 and over on July 1, 1998. Unpublished.

Nash, M. Nature’s Prozac? Time September 1997; 150: 80-82.

National Institutes of Health (NIH) Consensus Development Conference Statement: Diagnosis and Treatment of Depression in Late Life. 1991, Nov 4-6; 9(3):1-27.

Nature's RX. ABC 20/20. June, 1997 (Dr. Timothy Johnson)

Nielsen, BM et al. A comparison of fluoxetine and imipramine in the treatment of outpatients with major depressive disorder. Acta Psychiatry Scand 1993; 87: 269-72.

No Worry Wort. Prevention November, 1995; 47: 28-30.

Ormel, J, Koeter MW, Van der Brink W, Willige G. Recogintion, management and course of anxiety and depression in general practice. Archives of General Psychiatry $1991 ; 48: 700-6$.

Pande, AC and ME Sayler. Severity of Depression and Response to Fluoxetine. International Clinical Psychopharmacology. 1993; 8: 243-245.

Panel Recommends Primary Care Detection, Treatment of Major Depression. Public Health Reports. July/August, 1993; 108 (4): 524-526.

Patris, $\mathrm{M}$ et al. Citalopram versus fluoxetine: A double-blind, controlled, multicenter, phase III trial in patients with unipolar major depression treated in general practice. Internals of Clinical Psychopharmacology 1996; 16(2): 306-315.

SSRIs Among Top Therapeutic Class Prescribed. "The Pink Sheet" Prescription Pharmaceuticals and Biotechnology 2002; 64:22-23.

Poutaraud, A, Annelise Lobstein, Phillippe Girardin, and Bernard Weniger. Improved Procedure for the Quality Control of Hpericum perforatum L. Phytochemical Analysis 2001; 12: 355-362.

Powdered St. John's Wort extract monograph. Pharmacopoeial Forum of United State Pharmacopoeia (USP) 1999; 25(2).

Quandt, J, Schmidt, U, Schenk, N. Ambulante Behanlung leichter und mittelchwerer depressiver Verstimmunger. Der Allgemeinarzt 1993; 2: 97-102.

Rapaport, $\mathrm{M}$ et al. A comparison of fluvoxamine and fluoxetine in the treatment of major depression. Journal of Clinical Psychopharmacology. 1996; 11: 129-136. 
Rascati, K. Drug Utilization Review of Concomitant Use of Specific Serotonin Reuptake Inhibitors or Clomipramine with Antianxiety/Sleep Medications. Clinical Therapeutics 1995;17(4):786-790.

Rao, SG, Laxminarayana, AU, Saraswathi, LU, Padma, GM, Ganesh, R, Kulkarni, DR, Calendula and Hypericum: two homeopathic drugs promoting wound healing in rats.

Fitoterapia 1991; 6: 508-510.

Regier DA, Narrow WE, Rae DS, et al. The de facto mental and addictive disorders service system. Epidemiologic Catchment Area prospective 1-year prevalence rates of disorders and services. Archives of General Psychiatry 1993; 50(2): 85-94.

Reh C, Laux P. Hypericum extract in the treatment of depression: An effective alternative. Therapiewoche 1992; 42:1576-81.

Reports from the Canadian Coordinating Office for Health Technology Assessment (CCOHTA); Selective serotonin reuptake inhibitors for major depression. International Jounal of Technology Assessment in Health Care. 1998;14:394-6.

Revicki, DA. et al. Cost-Effectiveness of Newer Antidepressants Compared With Tricyclic Antidepressants in Managed Care Settings. Journal of Clincal Psychiatry February, 1997; 58(2): 47-58.

Reynolds, J. Martindale The Extra pharmacopoeia $30^{\text {th }}$ editionLondon: The Pharmaceutical Press: 1993.

Richardson, S and Allan Detsky. Users' Guides to the Medical Literature. Part VII. How to Use a Clinical Decision Analysis. B. What Are the Results and Will They Help Me in Caring for My Patients? JAMA May, 1995; 273: 1610-1613.

Rickels K, Schweizer E, Clary C et al: Nefazodone and imipramine in major depression: a placebo-controlled trial. British Journal of Psychiatry 1994; 164: 802-805.

Rioux et al. A double-blind comparison of nafazodone and fluoxetine in depressed patients. Psychopharmacology Bulletin 1996; 32: 505.

Schlich, D Braukmann, F, Schenk, N. Behandlung depressiver Zustandsbilder mit Hypericinium. Doppelblindstudie mit einem pflanzlichen Antidepressivum. Psycho 1987; 13: 440-445.

Schmidt, U, Sommer, H. Johanniskraut-Extrakt zur ambulanten Therapie der Depression. Fortschritte der Medizin 1993; 111:339-342.

Schrader E, Meier B, Brattstro"m A. Hypericum treatment of mild-moderate depression in a placebo-controlled study: a prospective, double-blind, randomized, placebocontrolled, multicenter study. Human Psychopharmacology. 1998;13:163-169. 
Schrader, E. Equivalence of St. John's Wort extract (Ze 117) and fluoxetine: a randomized, controlled study in mild-moderate depression. International Clinical Psychopharmacology 2000; 15(2): 61-68.

Shelton, RC et al. Effectiveness of St. John's wort in major depression. A randomized controlled trial. JAMA April 18, 2001;285: 1978-86.

Schulberg, HC and MR Block, MJ Madonia, et al. The 'Usual Care' of Major Depression In Primary Care Practice. Archives of Family Medicine. July/August, 1997; 6: $340-1$.

Sclar, D et al. Trends in the Prescribing of Antidepressant Pharmacotherapy: OfficeBased Visits, 1990-1995. Clinical Therapeutics 1998; 20 (4): 871-84.

Siegel, JE, Deaney, KM. Introduction to SMLTREE. Medical Decision Making 1993; $13: 74-84$.

Simon, G et al. Initial Antidepressant Choice in Primary Care. Effectiveness and Cost of Fluoxetine vs. Tricyclic Antidepressants. JAMA 1996; 275: 1897-1902.

Snaith, RP. Present Use of the Hamilton Depression Rating Scale: Observations on Method of Assessment in Research of Depressive Disorders. BMJ 1996, 168:594-7.

Snow, V, Steven Lascher, and Christel Mottur-Pilson. Pharmacologic Treatment of Acute Major Depression and Dysthymia. Clinical Guideline, Part 1. Annals of Internal Medicine. 2000; 132: 738-742.

Snow, J. Hypericum perforatum L. (Hyperiaceae). The Protocol Journal of Botanical Medicine. 1996; 2 (1): 16-21.

St. John's Wort. The Medical Letter November, 1997; 39 (1): 21

St. John's Wort vs. Tricyclic Antidepressants. American Journal of Natural Medicine. April, 1995; 2 (3): 8-17.

Stokes, P and Aliza Holtz. Fluoxetine Tenth Anniversary Update: The Progress Continues. Clinical Therapeutics 1997; 19: 1135-1226.

Thompson D, Buesching D, Gregor KJ, Oster G. Patterns of antidepressant use and their relation to costs of care. American Journal of Managed Care October 1996.

Thompson, T. Seeking The Wizards of Prozac. Saturday Evening Post. March, 1994; 266: 50-57. 
Tom, E and Kevin A. Schulman. Mathematical Models in Decision Analysis. Infection Control Hospital Epidemiology. 1997; 18: 65-73.

Turton-Weeks, Susan et al. St John's Wort: A Hidden Risk for Transplant Patients. Progress in Transplantation 2001;11(2):116-120.

Tylee, A and M Bowden, A Reynolds. A comparison of venlavaxine $37.5 \mathrm{mg}$ twice a day with fluoxetine $20 \mathrm{mg}$ once a day in depressed patients treated in general practice. Psychopharmacology Bulletin 1996; 32: 528.

Tyler, V. The Secrets of Saint-John's-Wort. Prevention February, 1997; 49: 74-78.

Undertreatment of Depression. American Family Physicians. April, 1997; 55 (5): 19882000 .

U.S. Preventive Services Task Force Now Finds Sufficient Evidence to Recommend Screening Adults for Depression. Press Release, May 20, 2002. Agency for Healthcare Research and Quality, Rockville, MD. http://www.ahrq.gov/news/press/pr2002/deprespr.htm

Vanderhoff, B and K Miller. Major Depression: Assessing the Role of New Antidepressants. American Family Physicians. January, 1997; 55 (1): 249-257.

van Gurp, G, Meterissian, GB, Haiek, LN, McCusker, J, Bellanvance, F. St. John's Wort or sertraline? A Randomized controlled trial in primary care. Canadian Family Physician 2002; 48: 905-912.

Vickery, AR. Traditional uses and folklore of Hypericum in the British Isles. Economic Botany 1981; 289-295.

Voltz, HP. Controlled Clinical Trials of Hypericum Extracts in Depressed Patients - An Overview. Pharmacopschiatry. 1997; 30 (Supplement); 72-76.

Volz, H, and Laux, P. Potential treatment for subthreshold and mild depression: A comparison of St. John's wort extracts and fluoxetine. Comprehensive Psychiatry. 2000; 41: 133-137.

Vorbach, EU and KH Arnoldt, WD Hubner. Efficacy and Tolerability of St. John's Wort Extract LI 160 Versus Imipramine in Patients with Severe Depressive Episodes According to ICD-10. Pharmacopsychiatry. 1997; 30 (Supp12): S81-S85.

Vorbach, EU, WD Hubner KH Arnoldt. Effectiveness and Tolerance of the Hypericum Extract LI 160 in Comparison with Imipramine: Randomized Double-Blind Study with 135 Outpatients. Journal of Geriatric Psychiatry and Neurology. 1994; 7 (suppl 1): 1923. 
Wagner, H and S Bladt. Pharmaceutical Quality of Hypericum Extracts. Journal of Geriatric Psychiatry and Neurology. October, 1994; 7 (supplement 1): 65-68.

Weinstein, MC and Fineberg, HV. Clinical Decision Analysis. Philadelphia: W.B. Saunders Company, 1980.

Werth W. Psychotonin M versus Imipramin in der Chriurgie. Der Kassenarzt 1989;15:64-8.

Wheatley, D. LI 160, an Extract of St. John's Wort, Versus Amitriptyline in Mildly to Moderately Depressed Outpatients - A Controlled 6-Week Clinical Trial.

Pharmacopsychiatry. 1997; 30 (Supplement): 77-80.

Whiskey E, Werneke U, Taylor D. A systematic review and meta-analysis of Hypericum perforatum in depression: a comprehensive clinical review. International Clinical Psychopharmacology 2001 Sep;16(5):239-52.

Wilke, M. SmithKline, AHP move into herbal remedies market. Advertising Age October, 1997; 68: 2-3.

Williams, JW. et al. A Systematic Review of Newer Pharmacotherapies for Depression in Adults: Evidence Report Summary. Annals of Internal Medicine 2000; 132: 743-756.

Woelk, H. Comparison of St. John's wort and impramine for treating depression: randomised controlled trial. BMJ 2000;321: 536-539.

Woelk, H and G Burkard, J Grunwald. Benefits and Risks of the Hypericum Extract LI 160: Drug Monitoring Study with 3250 Patients. Journal of Geriatric Psychiatry and Neurology. October, 1994; 7 (supplement 1): 34-38.

Workman, EA and DD Short. Atypical antidepressants versus imipramine in the treatment of major depression: A meta-analysis. Journal of Clinical Psychiatry. 1993; 54: 5-12. 


\section{Appendix A}

\section{Depression Treatment Algorithm Questionnaire}

The following questions were answered based on an average adult age 18 to 65 , with a first time diagnosis of major depression with mild to moderate severity who is treated with fluoxetine in the outpatient setting.

Responses from expert panel on treatment of depression

\begin{tabular}{|c|c|c|c|c|c|c|}
\hline PANEL MEMBER & 1 & 2 & 3 & 4 & 5 & 6 \\
\hline $\begin{array}{l}\text { 1. After the initial } \\
\text { diagnosis, do you } \\
\text { perform any baseline } \\
\text { tests? }\end{array}$ & $\begin{array}{l}\text { TSH } \\
\text { LEVEL, } \\
\text { CBC, LFT }\end{array}$ & $\begin{array}{l}\text { TSH } \\
\text { LEVEL }\end{array}$ & $\begin{array}{l}\text { TSH } \\
\text { LEVEL }\end{array}$ & $\begin{array}{l}\text { TSH, } \\
\text { CHEM-7 }\end{array}$ & TSH & NONE \\
\hline $\begin{array}{l}\text { 2. How do you assess or } \\
\text { diagnose depression? }\end{array}$ & DSM IV & DSM IV & DSM IV & BECK & DSM IV & DSM IV \\
\hline $\begin{array}{l}\text { 3. What is the starting } \\
\text { dose of Prozac you } \\
\text { would prescribe? }\end{array}$ & $\begin{array}{l}\text { 10MG X1 } \\
\text { WK, } \\
20 \mathrm{MG}\end{array}$ & $20 \mathrm{MG}$ & $20 \mathrm{MG}$ & $20 \mathrm{MG}$ & $\begin{array}{l}10 \mathrm{MG} \mathrm{X} \\
1 \mathrm{WK} \\
20 \mathrm{MG}\end{array}$ & $20 \mathrm{MG}$ \\
\hline $\begin{array}{l}\text { 4. When do you assess } \\
\text { response to therapy? }\end{array}$ & 4 weeks & 2-4 weeks & 3 weeks & 2 weeks & 2 weeks & $\begin{array}{l}2-4 \\
\text { weeks }\end{array}$ \\
\hline $\begin{array}{l}\text { 5. Are there any other } \\
\text { types of tests, such as } \\
\text { labs, that are performed } \\
\text { at this time? }\end{array}$ & $\begin{array}{l}\text { CBC, } \\
\text { LFTs }\end{array}$ & No & No & LFTs & No & No \\
\hline $\begin{array}{l}\text { 6. How many weeks of } \\
\text { therapy are necessary to } \\
\text { determine success or } \\
\text { failure on therapy? }\end{array}$ & 4 weeks & 4 weeks & 6 weeks & $\begin{array}{l}4 \text { to } 6 \\
\text { weeks }\end{array}$ & 2 to 4 weeks & 4 weeks \\
\hline $\begin{array}{l}\text { 7. What percentage of } \\
\text { patients (pts) respond to } \\
\text { fluoxetine by this time? }\end{array}$ & $\begin{array}{l}66.7 \% \\
(2 / 3)\end{array}$ & $60 \%$ & $\begin{array}{l}70 \% \text { to } \\
80 \%\end{array}$ & $50 \%$ & $60 \%$ & $\begin{array}{l}66.7 \% \\
(2 . / 3)\end{array}$ \\
\hline $\begin{array}{l}\text { 8. When a patient fails to } \\
\text { respond, what options } \\
\text { are considered? }\end{array}$ & $\begin{array}{l}\text { 个dose, } \\
\text { change Rx }\end{array}$ & $\begin{array}{l}\text { 个dose, } \\
\text { change } \mathrm{Rx}, \\
\text { add therapy }\end{array}$ & $\begin{array}{l}\text { 个dose, } \\
\text { change Rx }\end{array}$ & $\begin{array}{l}\text { 个dose, } \\
\text { change } \mathrm{Rx}\end{array}$ & $\begin{array}{l}\text { 个dose, } \\
\text { change } \mathrm{Rx}, \\
\text { add therapy }\end{array}$ & $\begin{array}{l}\text { 个dose, } \\
\text { change } \\
\mathrm{Rx}\end{array}$ \\
\hline $\begin{array}{l}\text { 9. What percentage of } \\
\text { patients requires an } \\
\text { increase in dosage? }\end{array}$ & $20 \%$ & $25 \%$ & $30 \%$ & $30 \%$ & $25 \%$ & $25 \%$ \\
\hline $\begin{array}{l}\text { 10. Once a response is } \\
\text { established, how often } \\
\text { does the patient return } \\
\text { for an assessment? }\end{array}$ & $\begin{array}{l}\text { Every } 2 \\
\text { months }\end{array}$ & $\begin{array}{l}\text { Every } 3 \text { to } 4 \\
\text { months }\end{array}$ & $\begin{array}{l}\text { Every } 3 \\
\text { months }\end{array}$ & Depends & $\begin{array}{l}\text { Every } 1 \text { to } 2 \\
\text { months } x 6 \\
\text { months }\end{array}$ & $\begin{array}{l}\text { Every } 3 \\
\text { to } 4 \\
\text { months }\end{array}$ \\
\hline
\end{tabular}


Depression Treatment Algorithm Questionnaire (cont'd)

\begin{tabular}{|c|c|c|c|c|c|c|}
\hline $\begin{array}{l}\text { 11. How long does the } \\
\text { average patient that } \\
\text { responds to Prozac } \\
\text { remain on the acute dose } \\
\text { of the drug? }\end{array}$ & 9 months & 12 months & $\begin{array}{l}6 \text { to } 12 \\
\text { months }\end{array}$ & $\begin{array}{l}6 \text { to } 9 \\
\text { months }\end{array}$ & $\begin{array}{l}6 \text { to } 12 \\
\text { months }\end{array}$ & $\begin{array}{l}12 \\
\text { months }\end{array}$ \\
\hline $\begin{array}{l}\text { 12. Is there a period after } \\
\text { the symptoms are } \\
\text { controlled that the dose } \\
\text { is decreased? }\end{array}$ & $\begin{array}{l}\text { Taper to } \\
50 \% \text { at } \\
\text { month } 8\end{array}$ & $\begin{array}{l}\text { Taper to } \\
50 \% \text { last } \\
\text { month }\end{array}$ & No & No & No & $\begin{array}{l}\text { Only if } \\
\text { pt } \\
\text { doesn't } \\
\text { tolerate } \\
\text { dose } \\
\end{array}$ \\
\hline $\begin{array}{l}\text { 13. What is the average } \\
\text { length of therapy for a } \\
\text { single depressive } \\
\text { episode? }\end{array}$ & 9 months & 12 months & $\begin{array}{l}6 \text { to } 12 \\
\text { months }\end{array}$ & 12 months & $\begin{array}{l}6 \text { to } 12 \\
\text { months }\end{array}$ & $\begin{array}{l}12 \\
\text { months }\end{array}$ \\
\hline $\begin{array}{l}14 . \text { What are the most } \\
\text { commonly reported } \\
\text { adverse drug reactions } \\
\text { (ADRs)? }\end{array}$ & $\begin{array}{l}\text { Edginess, } \\
\text { insomnia, } \\
\text { sexual } \\
\text { dysfxn. }\end{array}$ & $\begin{array}{l}\text { Jitteriness, } \\
\text { nervousness, } \\
\text { HA, GI } \\
\text { upset, } \\
\text { insomnia }\end{array}$ & $\begin{array}{l}\text { Nausea, GI } \\
\text { upset, } \\
\text { jitteriness, } \\
\text { sexual } \\
\text { dysfxn, HA }\end{array}$ & $\begin{array}{l}\text { Nausea, } \\
\text { jitteriness, } \\
\text { insomnia, } \\
\text { HA, sexual } \\
\text { dysfxn. }\end{array}$ & $\begin{array}{l}\text { Insomnia, } \\
\text { jitteriness, } \\
\text { nervousness, } \\
\text { nausea, } \\
\text { sexual } \\
\text { dysfxn. }\end{array}$ & $\begin{array}{l}\text { Insomnia } \\
\text { GI upset, } \\
\text { HA }\end{array}$ \\
\hline $\begin{array}{l}15 . \text { When do patients } \\
\text { most often complain of } \\
\text { ADRs? }\end{array}$ & Week 1-2 & $\begin{array}{l}\text { Week } 2 \text { and } \\
\text { on }\end{array}$ & Week 1-2 & $\begin{array}{l}\text { Within } 2 \\
\text { weeks }\end{array}$ & $\begin{array}{l}\text { First few } \\
\text { days }\end{array}$ & $\begin{array}{l}\text { Week 1- } \\
3\end{array}$ \\
\hline $\begin{array}{l}\text { 16. Of these, which most } \\
\text { often cause } \\
\text { discontinuation of the } \\
\text { drug? }\end{array}$ & Edginess & $\begin{array}{l}\text { Rarely cause } \\
\text { DC }\end{array}$ & $\begin{array}{l}\mathrm{HA}, \\
\text { jitteriness }\end{array}$ & $\begin{array}{l}\text { GI upset } \\
\text { and sexual } \\
\text { dysfxn. }\end{array}$ & $\begin{array}{l}\text { Nausea and } \\
\text { insomnia }\end{array}$ & $\begin{array}{l}\text { Rarely } \\
\text { cause DC }\end{array}$ \\
\hline $\begin{array}{l}\text { 17. What percentage of } \\
\text { people discontinue } \\
\text { Prozac because of } \\
\text { ADRs? }\end{array}$ & $20 \%$ & $\begin{array}{l}\text { Less than } \\
5 \%\end{array}$ & $20 \%$ & $5 \%$ & $10 \%-20 \%$ & $\begin{array}{l}\text { Less than } \\
1 \%\end{array}$ \\
\hline $\begin{array}{l}\text { 18. What, if any, } \\
\text { additional therapy do you } \\
\text { prescribe to treat ADRs? }\end{array}$ & $\begin{array}{l}\text { Trazodone } \\
\text { 50mg qhs }\end{array}$ & $\begin{array}{l}\text { Ativan 1mg } \\
\text { or trazodone } \\
50 \mathrm{mg} \text { qhs }\end{array}$ & $\begin{array}{l}\text { Trazodone } \\
50 \mathrm{mg} \text { qhs }\end{array}$ & $\begin{array}{l}\text { None, } \\
\text { Change } \\
\text { therapy }\end{array}$ & $\begin{array}{l}\text { Trazodone } \\
50 \mathrm{mg} \text { qhs }\end{array}$ & None \\
\hline $\begin{array}{l}\text { 19. What percentage of } \\
\text { patients require this } \\
\text { therapy? }\end{array}$ & $20 \%$ & $15 \%$ & $5-10 \%$ & $\begin{array}{l}5 \% \text { change } \\
\text { therapy }\end{array}$ & $10 \%$ & N/A \\
\hline $\begin{array}{l}\text { 20. When a patient } \\
\text { discontinues therapy due } \\
\text { to ADRs, what other } \\
\text { therapies do you try? }\end{array}$ & $\begin{array}{l}\text { Paxil } \\
\text { 20mg or } \\
\text { Zoloft } \\
50 \mathrm{mg}\end{array}$ & $\begin{array}{l}\text { Paxil 20mg } \\
\text { or Zoloft } \\
50 \mathrm{mg}\end{array}$ & $\begin{array}{l}\text { Paxil 20mg } \\
\text { or } \\
\text { Wellbutrin } \\
150 \mathrm{mg}\end{array}$ & $\begin{array}{l}\text { Wellbutrin } \\
150 \mathrm{mg}\end{array}$ & Paxil 20mg & $\begin{array}{l}\text { Another } \\
\text { SSRI }\end{array}$ \\
\hline $\begin{array}{l}\text { 21. Are there any ADRs } \\
\text { that require additional } \\
\text { medical care other than a } \\
\text { physician visit? }\end{array}$ & No & No & No & No & No & No \\
\hline
\end{tabular}


Depression Treatment Algorithm Questionnaire (cont'd)

\begin{tabular}{|c|c|c|c|c|c|c|}
\hline $\begin{array}{l}22 . \text { Estimate the average } \\
\text { number of physician } \\
\text { visits a patient has in } 1 \\
\text { year and for patients that } \\
\text { fail initial therapy. }\end{array}$ & $\begin{array}{l}5,6 \text { if fail } \\
1^{\text {st }} \text { therapy }\end{array}$ & 7 & $\begin{array}{l}6,7 \text { if fail } \\
1^{\text {st }} \text { therapy }\end{array}$ & 6 & $\begin{array}{l}6,7-8 \text { if fail } \\
1^{\text {st }} \text { therapy }\end{array}$ & $\begin{array}{l}5,6-7 \text { if } \\
\text { fail and } \\
\text { ADRs }\end{array}$ \\
\hline $\begin{array}{l}\text { 23. Do you refer (or } \\
\text { encourage) counseling } \\
\text { sessions for your } \\
\text { patients? }\end{array}$ & Yes & Yes & Yes & Yes & Yes & Yes \\
\hline $\begin{array}{l}\text { 24. Estimate the number } \\
\text { of counseling visits a } \\
\text { patient has in } 1 \text { year. }\end{array}$ & $4-6$ & 5 & $\begin{array}{l}\text { Depends on } \\
\text { insurance }\end{array}$ & 6 & 5 & 5 \\
\hline $\begin{array}{l}\text { 25. Are you familiar with } \\
\text { St. John's Wort and the } \\
\text { published trials? }\end{array}$ & $\begin{array}{l}\text { Yes, } \\
\text { listened to } \\
\text { seminar }\end{array}$ & No & Somewhat & Yes & $\begin{array}{l}\text { No, but } \\
\text { heard it has } \\
\text { some } \\
\text { positive } \\
\text { effects }\end{array}$ & No \\
\hline $\begin{array}{l}\text { 26. Do you prescribe the } \\
\text { herb? }\end{array}$ & No & No & No & No & No & No \\
\hline $\begin{array}{l}27 . \text { Are you aware of any } \\
\text { of your patients taking } \\
\text { the herb for depression? }\end{array}$ & $\begin{array}{l}\text { Yes, many } \\
\text { take on } \\
\text { their own }\end{array}$ & No & Yes & No & No & No \\
\hline $\begin{array}{l}28 . \text { Based on the } \\
\text { literature, do you feel the } \\
\text { herb is effective? }\end{array}$ & $\begin{array}{l}\text { Possibly } \\
\text { in mild to } \\
\text { moderate } \\
\text { depression }\end{array}$ & N/A & $\begin{array}{l}\text { Yes for } \\
\text { mild to } \\
\text { moderate } \\
\text { depression }\end{array}$ & $\begin{array}{l}\text { Not enough } \\
\text { information }\end{array}$ & $\mathrm{N} / \mathrm{A}$ & $\mathrm{N} / \mathrm{A}$ \\
\hline $\begin{array}{l}\text { 29. Would you prescribe } \\
\text { the herb based on } \\
\text { available data now } \\
(1998) ?\end{array}$ & No & No & No & No & No & No \\
\hline $\begin{array}{l}\text { 30. What information } \\
\text { would you like to see in } \\
\text { order to give you } \\
\text { confidence in prescribing } \\
\text { the herb? }\end{array}$ & $\begin{array}{l}\text { Standard- } \\
\text { ization } \\
\text { practices, } \\
\text { trials } \\
\text { against } \\
\text { standard } \\
\text { doses of } \\
\text { AD }\end{array}$ & $\begin{array}{l}\text { Regulation } \\
\text { by FDA, } \\
\text { long-term (6 } \\
\text { month) } \\
\text { trials, } \\
\text { comparisons } \\
\text { with SSRIs }\end{array}$ & $\begin{array}{l}\text { Regulation } \\
\text { by FDA } \\
\text { while } \\
\text { maintain } \\
\text { low cost }\end{array}$ & $\begin{array}{l}\text { More } \\
\text { rigorous } \\
\text { trials, } \\
\text { regulation } \\
\text { of products }\end{array}$ & $\begin{array}{l}\text { Studies } \\
\text { regarding } \\
\text { safety such } \\
\text { as drug } \\
\text { interactions }\end{array}$ & $\begin{array}{l}\text { Accepted } \\
\text { standard } \\
\text { of care } \\
\text { by MDs }\end{array}$ \\
\hline
\end{tabular}

KEY : AD = ANTIDEPRESSANT; ADR = ADVERSE DRUG REACTION;

$\mathrm{CBC}=\mathrm{COMPLETE} \mathrm{BLOOD} \mathrm{COUNT}$; CHEM-7 = SERUM BLOOD CHEMISTRY;

$\mathrm{DC}=$ DISCONTINUATION; DSM-IV = DIAGNOSTIC AND STATISTICAL MANUAL FOR

MENTAL DISORDERS-IV EDITION; GI = GASTROINTESTINAL; HA = HEADACHE;

LFT = LIVER FUNCTION TESTS; MD = MEDICAL DOCTOR; RX = TREATMENT;

TSH $=$ THYROID STIMULATIN HORMONE 


\section{Appendix B}

\section{Rolled Back Decision Tree}

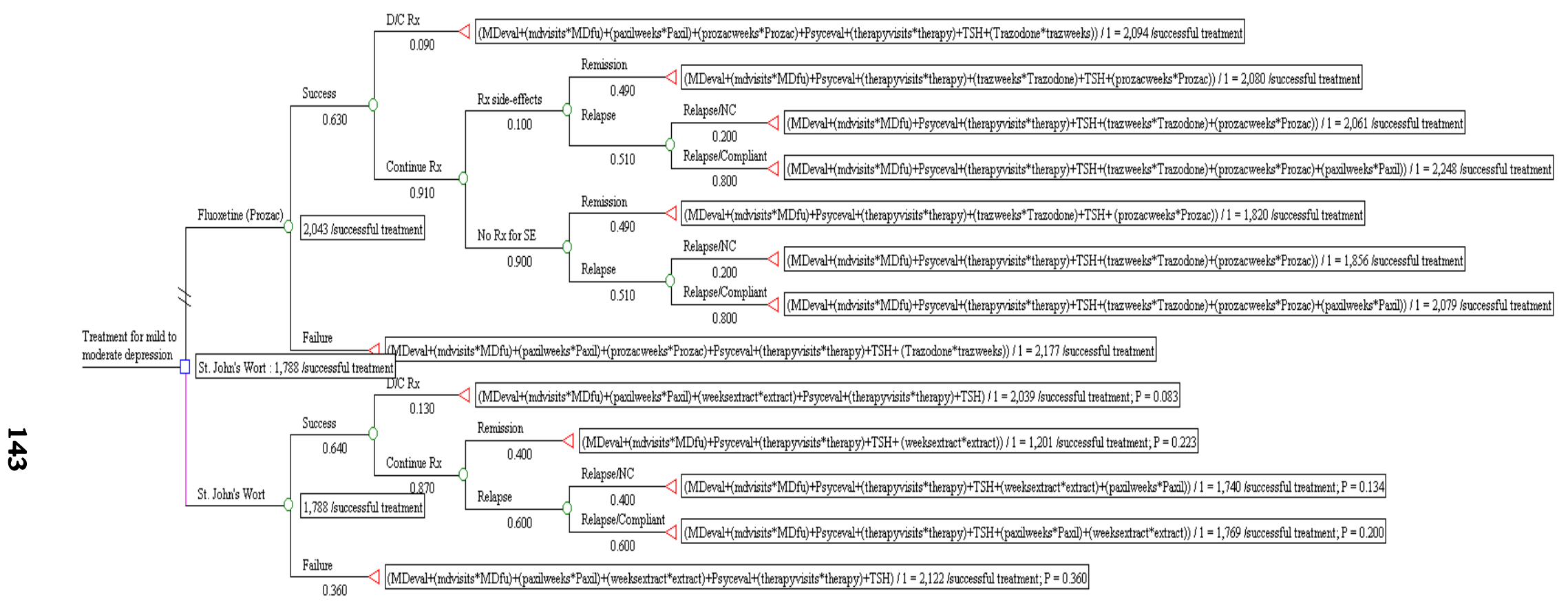

In the decision tree, it is revealed that initial treatment with St. John's Wort is the most cost-effective option compared to treatment with fluoxetine.

Mdeval $=$ cost of initial physician evaluation, $\mathrm{MDfu}=$ cost of physician follow-up visits, mdvisits $=$ total number of physician visits,

Psycheval = cost of initial psychotherapy evaluation, therapy = cost of psychotherapy session, therapyvisits = total number of psychotherapy sessions, Paxil $=$ weekly cost of Paxil, paxil weeks $=$ total number of weeks on Paxil, Prozac $=$ weekly cost of fluoxetine, prozacweeks $=$ total number of weeks on fluoxetine, extract = weekly cost of St. John's Wort, weeksextract = total number of weeks on St. John's Wort, TSH = cost of measurement of thyroid stimulating hormone serum level, Trazodone $=$ weekly cost of trazodone, trazweeks $=$ total number of weeks on trazodone, $\mathrm{D} / \mathrm{C} \mathrm{Rx}=$ discontinue initial therapy, $\mathrm{Rx}=$ treatment, $\mathrm{SE}=$ side-effects, $\mathrm{NC}=$ noncompliant 


\section{Lee Ann Thayer, RPh, MS}

478A Radmere Dr.

Cheshire, CT 06410

Email: thayerla@ynhh.org

Phone: (203) 699-0240

\section{Curriculum Vitae}

\section{Education}

Master of Science in Pharmaceutical Systems and Policy. Research Area: Pharmacoeconomics .West Virginia University May, 2003

Bachelor of Science in Pharmacy .West Virginia University December, 1995

\section{Professional Experience}

Yale-New Haven Hospital, New Haven, CT

August, 2000 to Present

\section{Clinical Specialist, Drug Utilization}

Y-NHH is a 944-bed tertiary care, academic medical center with an innovative decentralized pharmacy department.

- Drug use management-coordinate the process for reviewing non-formulary (NF) medication requests, successfully implemented a process that decreased use of NF drugs by $97 \%$, train pharmacy practice residents to evaluate and respond to NF requests via a 24 hour beeper service, serve as clinical advisor to the P\&T Committee, evaluate new drug requests, develop drug use policies, and conduct drug utilization reviews

- Budget preparation and new technology projections- evaluate and implement pharmacy initiatives to improve patient care and reduce pharmacy costs, project the clinical and fiscal impact of new technology to enable adequate fiscal planning

- Oversight of the Drug Information (DI) Center- train the DI resident, coordinate and edit monthly hospital publications, and manage drug shortages

- Training and education- present ACPE lectures to medical, pharmacy, and nursing staff, develop and implement pain management protocols, serve as primary preceptor to the drug information resident, serve as primary advisor to staff pharmacists for review of pharmaceutical care conference lectures, contribute to the department's external academic involvement

\section{Yale New Haven Hospital, New Haven, CT}

November, 1998 to August, 2000

\section{Clinical Pharmacist}

Experience in general medicine and surgery with an emphasis on critical care. Focus was on promoting rational drug use and ensuring patient safety.

- Evaluated patient profiles and intervened on dosing and drug selection

- Promoted a pro-active role by participating in patient care rounds specifically in the surgical and medical intensive care units

- Provided in-services to the medical/pharmacy staff and disseminated drug information through hospital publications and presentations

\section{City Pharmacy, Morgantown, WV}

\section{December, 1996 to October, 1998}

\section{Staff Pharmacist}

An independent community pharmacy that provides pharmaceutical care services including medication use counseling, drug information, durable medical equipment, and specialized compounding.

- Reviewed patient profiles monitoring safety and appropriateness of drug therapy

- Performed interventions regarding appropriate dosing and selection of drug therapy

- Counseled patients and families on proper use of prescription and over-the-counter medications and provided additional information regarding disease states and general health issues 


\section{Professional Experience (cont'd)}

West Virginia Bureau for Medical Services, Charleston, WV

Summer Semester Graduate School, 1997

Research Assistant: WV Medicaid

WV Medicaid has a pharmacist-run formulary system that utilizes a prior-authorization program for management of many therapeutic drug classes.

- $\quad$ Aided in the development of the WV Medicaid Provider's Manual for Home Parenteral Therapy that included billing and reimbursement policies and requirements to be certified as a Medicaid Provider

- $\quad$ Created the inspection guidelines and documentation forms for Home IV Pharmacy providers

West Virginia University School of Pharmacy, Morgantown, WV

Fall Semester Graduate School, 1997

Teaching Assistant: Pharmacy Communications

- Lectured and facilitated the recitation section of the Communications class for second-year pharmacy students

- Aided in the development of course materials, graded class projects such as journals, papers, and videotapes on counseling skills

West Virginia University School of Pharmacy, Morgantown, WV

First Year Graduate School, 1996

Research Assistant: WVU Pharmacy Immunization Project (PIP)

PIP is a federally funded study to develop the pharmacists' role in childhood and adult immunizations through the creation, implementation, and evaluation of a pharmacy-based immunization service.

- Developed instruments to measure current rates, attitudes, and awareness of immunizations in the study and control populations

- $\quad$ Supervised the conduction of the telephone survey of over 900 mothers in WV, coded and analyzed data in Lotus Approach and SPSS

- $\quad$ Responsible for the marketing campaign including development and distribution of promotional materials such as flyers, newspaper and radio advertisements, created pharmacy and nursing data collection forms to capture patient and clinical information

- Served as liaison for the participating pharmacies, county health departments, and WVU School of Pharmacy.

Mylan Pharmaceuticals, Inc., Morgantown, WV

July, 1993 to January, 1995

Chemist

Mylan is a leading generic pharmaceutical manufacturer.

- Worked in Quality Control lab preparing drug samples for purity testing using techniques such as dissolution tests, composite assays, and uniformity tests for High Pressure Liquid Chromatography (HPLC) analysis

- $\quad$ Recorded and analyzed resulting data

\section{Podium and Poster Presentations}

"Evaluating the Cost-Effectiveness of St. John's wort compared to fluoxetine for the treatment of depression" Masters Thesis Defense, West Virginia University School of Pharmacy, December 2002.

"Pain Management: Involving the Pharmacist" ACPE accredited lecture presented to Y-NHH pharmacy staff, October 2002.

"Pharmacoeconomics of Antibiotic Therapy" Bi-annual Lecture, Y-NHH Consultant Pharmacists Traineeship (The ICPS group), December 2001-August, 2002.

"Pain Management: Lessons Learned In Implementation" Presented at VHA Northeast Clinical Advantage Program in Pain Management, November, 2001. 


\section{Podium and Poster Presentations (cont'd)}

"Improving Pain Management" Poster Presentation, Y-NHH Quality Conference, October 2001.

"Principles of Pharmacokinetics" Lecture, Yale School of Nursing Graduate Program, September 2001.

"Improving Medication Safety: Meperidine Guidelines" Presented to Y-NHH Pharmacy and Therapeutics Committee, May 2001.

"Formulary Management: Engaging the Medical Staff" Presented to Visiting Spanish Scholars Program, Yale School of Medicine, March 2001.

"Use of Vasopressors in Septic Shock." Presented to Pharmacy Staff at Y-NHH Pharmaceutical Care Conference, March 2000.

"Treatment of Vancomycin-Resistant Enterococcus (VRE)." Presented at Y-NHH Surgical Intensive Care Unit (SICU) Conference, January 2000.

"Antimicrobial Management of Acute Exacerbations of Chronic Bronchitis." Presented to Pharmacy Staff at Y-NHH Pharmaceutical Care Conference, May 1999.

Madhavan S, Rosenbluth SA, Thayer LA, Odedina FT, Amonkar M, Richards T, Ambegaonkar A. "Does Immunization Provider Site Influence Completion Rates and Perceptions of Quality and Satisfaction with Immunization Service.” Poster Presentation, APhA's National Immunization Conference, July 1998.

\section{Committee Membership at $\mathrm{Y}-\mathrm{NHH}$}

Pharmacy and Therapeutics Committee, Clinical Advisor 2000-present

- critically evaluate requests to the formulary, drug use policy, and medication safety

- make recommendations on drug use based on clinical, safety, and economic reviews

Medical ICU Sedation and Analgesia Committee 2001-present

- created algorithm for prescribing sedation/analgesia in the MICU

- inservicing physicians, pharmacists, and nurses on use of sedatives and analgesics

Anticoagulation Subcommittee, Clinical Advisor 2001-present

- evaluated low molecular weight heparin class to determine most cost-effective formulary agent

- developing protocol for DVT prophylaxis

- $\quad$ evaluating treatment for HIT

Pain Management Prescribing Group 2001-present

- created a Pain Management Pocket Reference

- developing guided pathways for pain management in the computerized physician order entry (CPOE) system

Nursing Pain Council 2001-present

- created policies for assessment and documentation of pain

- provide education to nurses and medical staff on the use of analgesics

Nursing Critical Care Council 2001-present

- evaluate clinical management pathways and nursing policies

Cardiothoracic ICU Quality Conference Committee 2000-2001

- developed guidelines for use of rofecoxib for preemptive analgesia 


\section{Appointments}

Yale University, Adjunct Clinical Professor 2001-present

School of Nursing, Graduate Nursing Program

New Haven, CT

University of Connecticut, Adjunct Clinical Professor 1999-present

School of Pharmacy

Storrs, CT

\section{Process Improvement/Change Management Training}

General Electric (GE) Change Acceleration Process (CAP) training

GE Workout ${ }^{\mathrm{TM}}$ training

\section{Professional Organizations}

Connecticut Society of Hospital Pharmacists (CSHP)

American Society of Health Systems Pharmacists (ASHP)

American Pain Society (APS)

International Society of Pharmaceutical Outcomes Research (ISPOR)

Professional Licensure

1996 to Present

1998 to Present
Registered Pharmacist by examination in West Virginia

Registered Pharmacist by reciprocity in Connecticut 